\title{
34. PROVENANCE AND MATURITY OF ORGANIC CARBON IN LATE TERTIARY TO QUATERNARY SEDIMENTS FROM THE TYRRHENIAN SEA- (ODP LEG 107/HOLES 652A AND 654A) ${ }^{1}$
}

\author{
Kay-Christian Emeis, ${ }^{2}$ Bernd Mycke, ${ }^{3,4}$ and E. T. Degens ${ }^{3}$
}

\begin{abstract}
We analyzed samples from ODP Holes 652A and 654A (Leg 107, Tyrrhenian Sea) for the amount, type, and thermal maturity of organic matter. The sediments encompass clastic and biogenic lithologies, which were deposited on the passive margin east of Sardinia since the late Miocene to the Pleistocene. Marine, hypersaline/evaporitic, lacustrine/riverine, and finally hemipelagic marine conditions with occasional anoxic(?) interludes gave rise to very diverse sedimentary facies. The majority of samples is lean in organic matter $(<0.2 \%$ TOC). Notable exceptions are Tortonian sediments (TOC average 0.3\%), Messinian oil shales from Core 107-652A-64R (up to $11 \%$ TOC), Messinian lacustrine/fluvial sediments from Hole $652 \mathrm{~A}$ (TOC average $0.42 \%$,), and Pleistocene sapropel samples ( $>2 \%$ TOC). The Messinian oil shale in Hole $652 \mathrm{~A}$ appears to be the only mature hydrocarbon source rock. In general, Pliocene sediments are the leanest and least mature samples. Pleistocene and Pliocene samples derive organic matter from a marine source. In spite of obvious facies differences in the Messinian between the two sites, pyrolysis results are not conclusive in separating hypersaline facies of Site 654 from the fresh water facies of Site 652, because both appear to have received terrestrial organic tissue as the main component of TOC. It is apparent from the distribution of maximum pyrolysis temperatures that heat flow must have been considerably higher at Site 652 on the lower margin in the Messinian.

Molecular maturity indices in lipid extracts substantiate the finding that the organic matter in Tortonian and Messinian samples from Hole 654A is immature, while thermal maturation is more advanced in coeval samples from Hole $652 \mathrm{~A}$. Analyses of lipid biomarkers showed that original odd-even predominance was preserved in alkanes and alkylcyclohexanes from Messinian samples in Hole 654A, while thermal maturation had removed any odd-even predominance in Hole 652A. Isomerization data of hopanes and steranes support these differences in thermal history for the two sites. Hopanoid distribution further suggests that petroleum impregnation from a deeper, more mature source resulted in the co-occurrence of immature and mature groups of pentacyclic biomarkers.

Even though the presence of 4-methylsteranes may imply that dinoflagellates were a major source for organic matter in the oil shale interval of Hole 652, we did not find intact dinoflagellates or related nonskeletal algae during microscopic investigation of the organic matter in the fine laminations. Morphologically, the laminations resemble bacterial mats.
\end{abstract}

\section{INTRODUCTION, GEOLOGICAL BACKGROUND, AND OBJECTIVES}

The Tyrrhenian Sea is a small $\left(<260,000 \mathrm{~km}^{2}\right)$ and young $(<6 \mathrm{Ma})$ oceanic basin within the complex tectonic framework of the Mediterranean Sea. This youngest of the Mediterranean sub-basins evolved as a back-arc basin during subduction of eastern Mediterranean oceanic crust behind the Calabrian volcanic arc (Rehault et al., 1987). It is bounded to the west by the extended and thinned continental margins of Sardinia and Corsica, to the south by Sicily, and to the east and north by mainland Italy (Fig. 1).

\section{Results of Drilling at Sites 652 and 654}

In the following paragraphs, results of drilling at Sites 652 and 654 are briefly recounted from the Site Reports given in Kastens, Mascle, et al. (1987). Some interpretation of faciesadopted mainly from Robertson et al. (this volume)-are presented as the geological background for this study on organic matter sedimentation on the Sardinian margin since the late Miocene.

\footnotetext{
${ }^{1}$ Kastens, K. A., Mascle, J., et al., 1990. Proc. ODP, Sci. Results, 107: College Station, TX (Ocean Drilling Program).

2 Ocean Drilling Program, Texas A\&M University, 1000 Discovery Drive, College Station, TX 77843.

3 Geologisch-Paläontologisches Institut und Museum, Universität Hamburg, Bundesstrasse 55, D-2000 Hamburg 13, Federal Republic of Germany.

${ }^{4}$ Present address: Institut de Chimie, Université Louis Pasteur de Strasbourg, 1, Rue Blaise Pascal, 67008 Strasbourg Cedex, France.
}

\section{Site 652}

Site $652\left(40^{\circ} 21.30^{\prime} \mathrm{N}\right.$ and $\left.12^{\circ} 08.59^{\prime} \mathrm{E}\right)$ is situated in $3446 \mathrm{~m}$ water depth on the lowermost Sardinian margin in an elongated, north-trending half-graben. The sediments in this halfgraben basin are tilted to the west, and the basin appears to be bordered by seaward-dipping listric normal faults. The morphology and seismic images of the basin suggested that the basin was formed by rotational normal faulting of the margin and that sediments were deposited during pre-, syn-, and post-rift stages of margin evolution.

Five sedimentary units were recognized in Hole $652 \mathrm{~A}$ that reached a total depth of $721.1 \mathrm{~m}$, but fell short of recovering unambiguous pre-rift strata (Fig. 2).

Sedimentary Unit I (0-55 mbsf) is of Pleistocene age and consists of gray calcareous muds and gray muds, commonly containing volcanic glass and ash. Sedimentary Unit II (55-188 mbsf) ranges from the lower Pleistocene to Pliocene in age. Sediments of this unit consist of marly nannofossil oozes. The base of the unit coincides with the Miocene/Pliocene boundary (i.e., the change in environment at the end of the Messinian). Sedimentary Unit III (188-189 mbsf) of uppermost Messinian age is a transitional interval of lively colored (red, brown, gray, and mixtures of the above) muds and clays between the normal marine Pliocene sediment of sedimentary Units II and IV. Sedimentary Unit IV (189-345 mbsf) is largely barren of diagnostic microfossils, but is assumed to be late Messinian in age. The sediments are dominated by gray, thinly bedded gypsum- and carbonate-bearing sandy mud, tentatively assigned a turbiditic origin. Sedimentary Unit V (345-721 mbsf) was barren of dat- 


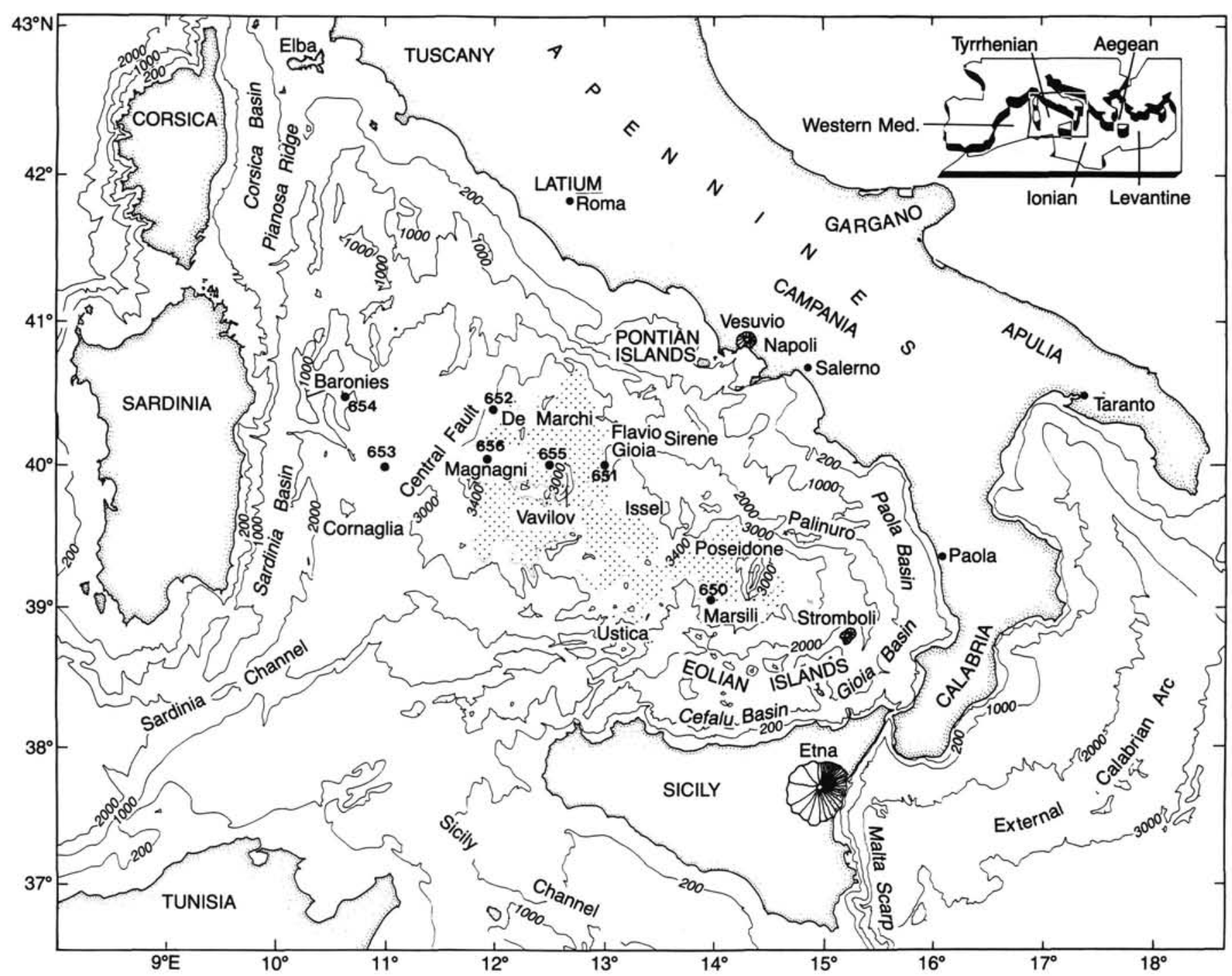

Figure 1. Location map of ODP Leg 107 drill sites in the Tyrrhenian Sea. Site 652 and 654 are located on the lower and upper Continental margin of Sardinia, respectively. From Kastens, Mascle, et al., 1987.

able fossils, but was probably deposited during the Messinian. The interval from 345 to 684 mbsf consists of gray to dark gray sandy muds, which are normally graded, gypsum-, carbonate-, and plant-debris-bearing. An interval of $0.5 \mathrm{~m}$ thickness in Core 652A-64R contained a thin oil-shale bed of high organic carbon concentrations impregnated by petroleum. The basal thick $(538.2 \mathrm{~m})$ Messinian succession is comprised of almost completely barren clastic and chemical sediments, which Robertson et al. (this volume) interpret as a series of either lacustrine or coastal plain deposits.

It appears that the depositional environment of the Messinian succession recovered in Hole 652A was a brackish, at times stagnant, water body on a rapidly subsiding coastal plain within the range of occasional marine ingressions. Graded clastic sediments of this facies were derived from fluvial systems or from settling of suspensates in ephemeral lakes or lagoons that fluctuated between fresh-water, brackish, and hypersaline conditions.

\section{Site 654}

Site $654\left(40^{\circ} 34.76^{\prime} \mathrm{N}\right.$ and $10^{\circ} 41.80^{\prime} \mathrm{E}$, see Fig. 1) is situated in $2208 \mathrm{~m}$ water depth on the upper continental margin of Sardinia in a fault-bounded, roughly north-trending half-graben. Geometry of reflectors in seismic profiles suggested that sedi- ments in the basin are comprised of pre-, syn-, and post-rift sediments.

Six sedimentary units were recognized in Hole 654A, which reached a total depth of $\mathbf{4 7 3 . 6}$ mbsf and ended in Tortonian(?) or pre-Tortonian clastic and conglomeratic sediments (Fig. 3).

Sedimentary Unit I (0-242.7 mbsf) is of Pliocene and Pleistocene age and consists of nannofossil oozes with subordinate calcareous muds, volcanic ashes, and 10 individual sapropels and sapropelic intervals. Sedimentary Unit II (242.7-312.6 mbsf) is composed of clastic and chemical sediments deposited during the Messinian. Massive gypsum is interbedded with calcareous clay and claystone, mudstone, dolostone, anhydrite and rare sandstone, and nannofossil chalk. Dominated by finely laminated, organic-rich clastic sediments, sedimentary Unit III (312.6-348.9 mbsf) of probable Messinian age contains claystones, calcareous siltstones, and minor volcanic glass. Sedimentary Unit IV (348.9-403.9 mbsf) comprises burrowed nannofossil oozes of Tortonian to early Messinian age. The inferred age of Units I-IV is earliest Messinian, Tortonian, or older.

Following the analysis of Robertson et al. (this volume), the succession of sedimentary facies at Site 654 on the upper Sardinian margin evolved from basal coarse-grained clastic deposits of sedimentary Unit VI that were deposited as an alluvial fan on 


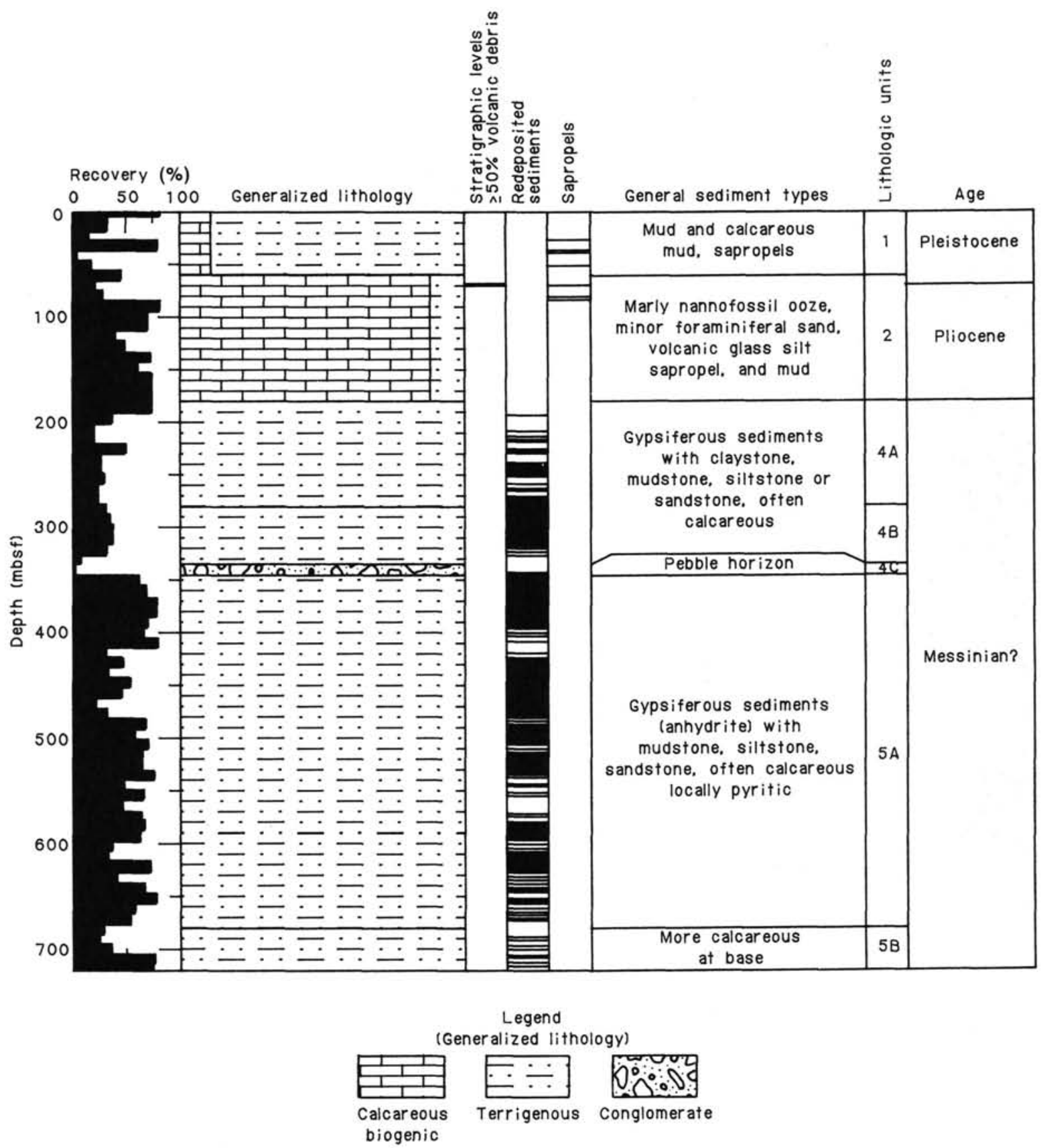

Figure 2. Lithologic summary column and facies of Site 652 sediments. From Kastens, Mascle, et al., 1987.

top of continental, possibly metamorphic, basement. The preMessinian strata are a good example of transgressive marine sediments depositing in a deepening basin after flooding of continental crust. Coinciding with the onset of desiccation and lowering of sea level in the early Messinian, the sedimentary facies at Site 654 changes to dark, finely laminated and organic carbon-rich clastic sediments that are intercalated with calcareous and dolomitic layers. The well-preserved lamination, the occurrence of siliceous microfossils and fish debris, and the abundance of marine-derived organic matter indicate that the sediments were deposited in a fertile marginal sea, possibly under conditions of localized upwelling. Laminations indicate that the sediments and bottom water were temporarily anoxic. A similar facies was described in the Tripolis Formation of the Caltanissetta Basin on Sicily by Broquet et al. (1984). The evaporitic se- ries of sedimentary Unit II are interpreted as clastic and chemical deposits in an evaporitic environment that was considerably deeper and more distal than that encountered at Site 652. At Site 654 , the Messinian depocenter did not experience fresh-water or brackish ingressions. Pliocene transgression established marine hemipelagic sedimentation and nannofossil marls and oozes with admixtures of volcaniclastic sediments are dominant sediments deposited through the Pleistocene. Noteworthy is the occurrence of 10 discrete sapropels and sapropelic intervals in the marine sediments of sedimentary Unit 1 .

This article attempts to characterize the evolution of depositional and postdepositional conditions for organic matter preservation and maturation through the stages of passive margin evolution since the Tortonian. Each of the various depositional environments outlined above are expected to give rise to char- 


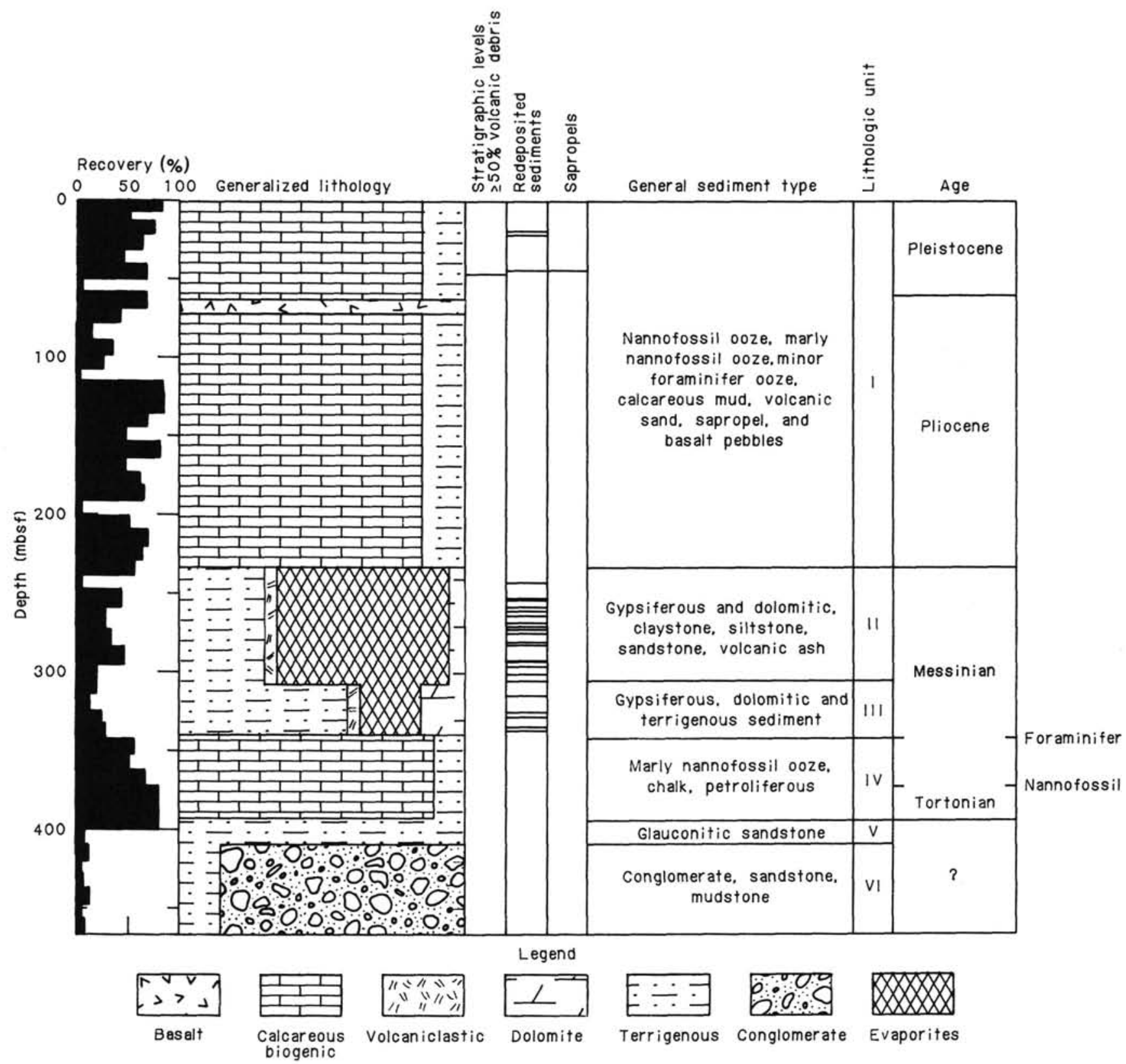

Figure 3. Lithologic summary column and facies of Site 654 sediments. From Kastens, Mascle, et al., 1987.

acteristic conditions for organic matter sedimentation and the amount or the quality of TOC buried. Questions that will be highlighted and discussed include the following main topics: (1) Is the evolution of the sedimentary facies paralleled by changes in the type and abundance of organic matter preserved? (2) Does the type of organic matter help in reconstructing unfossiliferous and ambiguous depositional environments deposited during the Messinian salinity crisis? (3) Can general rules for patterns of organic matter deposition on nascent passive margin be derived? and (4) What is the petroleum potential on the passive margin of Sardinia?

\section{SAMPLES AND METHODS}

Samples were taken on board the JOIDES Resolution. In most cases, the cores had equilibrated thermally for approximately $3 \mathrm{hr}$ and were split according to standard ODP procedure. In order to avoid possible contamination of the samples with debris of core liner, the surface of the working half was cleaned with a spatula. Sample size for pyrolysis and organic carbon determinations was $5 \mathrm{~cm}^{3}$ of sediment; for lipid analysis and microscopy, $40 \mathrm{~cm}^{3}$ were taken. Samples were immediately freeze dried, ground in an agate mortar rinsed with analytical-grade solvent, and stored in equally rinsed glass bottles.

\section{Organic Carbon Determinations and Pyrolysis}

Rock-Eval pyrolysis (Espitalié et al., 1985) was used to determine the amount, type, and maturity of organic carbon present in the samples. The instrument (Delsi Nermag Rock-Eval II with TOC module) measures five parameters, while a microprocessor-controlled temperature program heats the sample from $300^{\circ}$ to $600^{\circ} \mathrm{C}$ in an inert atmosphere. The hydrocarbons present in a sample are liberated initially during isothermal heating at $300^{\circ} \mathrm{C}$ and are recorded as $\mathrm{S}_{1}$ (mg hydrocarbons/g rock). Hydrocarbons produced by pyrolysis of kerogen in the sample are recorded as $\mathrm{S}_{2}$ (mg hydrocarbons/g rock), their amount being dependent on the type and maturity of the kerogen. The temperature at which most of the kerogen is pyrolyzed (coinciding with the maximum in $\mathrm{S}_{2}$ ) is recorded as $T_{\text {max }}$, a measure of organic matter maturity. $S_{1}$ and $S_{2}$ are measured with a flame ionization detector (FID) against a standard. $\mathrm{CO}_{2}$ is trapped during measurement of $\mathrm{S}_{1}$ and $\mathrm{S}_{2}$ at temperatures be- 
tween $300^{\circ}$ and $390^{\circ} \mathrm{C}$ and is recorded by a thermal conductivity detector (TCD) as $\mathrm{S}_{3}$. Experiments with several carbonate minerals (calcite, dolomite, siderite, ankerite) showed that $\mathrm{S}_{3}$ is unaffected by liberation of $\mathrm{CO}_{2}$ at these temperatures from decarboxylation of carbonates (Emeis, unpubl. data). In a second oven the remaining TOC is combusted to $\mathrm{CO}_{2}$. This and the carbon measured as $\mathrm{S}_{1}$ and $\mathrm{S}_{2}$ are used to calculate total organic carbon (TOC) content of the sample.

The Hydrogen Index (HI, $\left.100 \times \mathrm{S}_{2} / \mathrm{TOC}\right)$ and Oxygen Index (OI, $\left.100 \times \mathrm{S}_{3} / \mathrm{TOC}\right)$ are pyrolysis parameters that are frequently used in a way similar to the traditional elemental analysis of kerogen and coal (Espitalié et al., 1985; van Krevelen, 1961). A conservative interpretation is necessary in cases when these derived parameters are used to reconstruct provenance of the organic matter (kerogen type) in immature organic matter from shallow to intermediate (roughly 0-400 mbsf) depth, because labile components may be abundant and may shift the $\mathrm{HI}$ and OI of this organic matter significantly. Adsorption phenomena of hydrocarbons on clastic components of the sediments may further result in incomplete pyrolysis yield at low TOC concentrations (Katz, 1983). In these cases, traditional maturation and genetic pathways do not apply. Because of artifacts introduced by calculating $\mathrm{OI}$ and $\mathrm{HI}$ for samples of low organic carbon content $(<0.2 \%)$ and low signal-to-noise ratio, oxygen indices of these samples are very high and have not been plotted. They are listed in the data tables in cases of TOC $>0.1 \%$. In order to alleviate the problem of erroneously high oxygen indices and of errors introduced by high oxygen contents of immature organic matter, data plotted here relate $\mathrm{HI}$ and $\mathrm{T}_{\max }$ to provenance and maturity.

\section{Lipid Analyses}

Lipid analyses in shore-based studies employed the following techniques: Dried sediment samples were extracted with toluene/methanol (3:1) in a soxhlet extractor for $24 \mathrm{hr}$. After solvent evaporation the total extract was weighed without correction for co-extracted sulfur. A fraction of the extract was subjected to column chromatography on $20 \times$ $2 \mathrm{~cm} \mathrm{SiO}{ }_{2}$. Elution with petroleum ether yielded the total hydrocarbon fraction. Isoprenoid hydrocarbons were obtained by addition of the $n$ alkanes onto a $5 \AA$ molecular sieve. Desulfurization was attempted with two samples by adding the hydrocarbon fraction to washed and activated elemental copper. Because we noted significant loss of hydrocarbons during this procedure, we eliminated this step from our experiment in subsequent samples.

Samples were run on a Carlo Erba HRGC Model 4160 equipped with on-column injection and FID. The hydrocarbons were separated on a DB- 5 fused silica column $(30 \mathrm{~m} \times 0.2 \mathrm{~mm})$ during a temperature program with $5 \mathrm{~min}$ isothermal temperature at $80^{\circ} \mathrm{C}$, ramping to $300^{\circ} \mathrm{C}$ at $3^{\circ} / \mathrm{min}$, followed by $10 \mathrm{~min}$ isothermal heating at $300^{\circ} \mathrm{C}$. Gas-chromatography/mass spectroscopy was run on a Varian CH7A MS coupled with a Carlo Erba HRGC of the same specifications and run under same conditions as noted above. Mass spectrometer conditions were an ionizing energy of $70 \mathrm{eV}$, source temperature of $250^{\circ} \mathrm{C}$, and a scan speed of $1.3 \mathrm{~s} / \mathrm{dec}$. Molecular structures were identified by comparison with literature data and computerized library search.

\section{UV Microscopy}

Polished blocks of Messinian oil shale imbedded in resin were investigated by reflected-light fluorescence microscopy in order to discern the dominant type of organic matter. Photographs were taken with a Zeiss Universal photomicroscope equipped with UV light source and filter $365 / 395 / 420$.

\section{RESULTS AND DISCUSSION}

\section{Organic Carbon}

Organic carbon concentrations of samples from Holes $652 \mathrm{~A}$ and $654 \mathrm{~A}$ are listed as TOC in Tables 1 and 2 and are shown in depth plots in Figures 4 and 5, where lithologic unit boundaries are indicated by dashed lines. For ages of the lithologic units refer to Figures 2 and 3 . As can be seen in the plots of organic carbon concentration vs. depth in the two holes (Figs. 4 and 5), the sections recovered in Holes 652A and 654A show considerable similarities in concentrations of TOC. Influence of the depositional environment is particularly obvious in the Pliocene and Pleistocene sapropels and sapropelic sediments compared with the low TOC concentrations found in the surrounding hemipelagic calcareous muds. Accumulation rates and TOC values change abruptly to values several times higher in these supposedly anoxic sediments (Emeis, unpubl. data).

At Site 652 , we recognize highly variable organic carbon concentrations at the top of the Messinian sequence around 200 mbsf. Lithologic Subunit IVa (188.6-286.3 mbsf), however, is generally low in TOC $(<0.2 \%)$, while values in Unit $\mathrm{V}$, the authigenic gypsum-bearing, cyclic sequence, steadily increase downsection until they reach an absolute maximum $(11 \% \mathrm{TOC})$ in an oil shale layer in Core 107-652A-64R at 605 mbsf. This interesting oil shale facies will be treated in more detail below. It is not possible to attribute the observed high organic carbon concentrations of lithologic Unit Va to either productivity or preservation of increased amounts of terrestrial material, because accumulation rates cannot be calculated for lack of biostratigraphic age determinations.

Gypsiferous sediments deposited in sedimentary Unit 11 (242.7-312.6 mbsf) of Hole 654A age are believed to be of Messinian age. It is surprising that TOC values in the sediment never exceed $0.27 \%$ (Table 2, Fig. 5), even though equivalent Holocene hypersaline environments frequently show high productivity coupled with excellent preservation of sedimentary organic matter (e.g., Klok et al., 1984). Lower to middle Messinian sediments of sedimentary Unit III (represented by Samples $107-654 \mathrm{~A}-37 \mathrm{R}-1,75-76 \mathrm{~cm}$, to $107-654 \mathrm{~A}-40 \mathrm{R}-5,65-67 \mathrm{~cm}$, in Table 2) are laminated and reach up to $3.21 \%$ organic carbon. They show a definite trend to higher hydrogen indices (see below) and may be of algal origin. Tortonian (lower Messinian?) sediments of sedimentary Unit IV average $0.32 \%$ TOC and show very little scatter in the concentrations. The origin of organic matter in these sediments is algal marine, a fact that becomes apparent in pyrolysis and lipid analyses of extracts as discussed below.

\section{Origin and Maturity of Organic Matter from Whole Rock Pyrolysis}

Figures 6 and 7 show the hydrogen indices of samples from Holes $652 \mathrm{~A}$ and $654 \mathrm{~B}$ plotted against $\mathrm{T}_{\max }$ of pyrolysis (data listed in Tables 1 and 2). In Figure 6, the traditional evolution paths of organic matter of Type 1 (high HI: brackish organic matter), Type II (intermediate HI: marine organic matter), and Type III (low HI: terrestrial organic matter) are indicated by dashed lines, and empirical vitrinite reflectance boundaries of $0.5 \% R_{0}$ and $1.3 \% R_{0}$ are denoted by solid lines. Average values for $\mathrm{T}_{\max }$ and $\mathrm{HI}$, as well as ranges and standard deviations of samples of all age units, are plotted in Figure 7 for easy reference.

Facies patterns, preservation effects, and shifts in maturity are readily discernible in both sets of figures. Tortonian sediments at Site 654 with considerable organic carbon concentrations are for the most part thermally immature. They cluster in an area of the $\mathrm{HI} / \mathrm{T}_{\max }$ diagram that is typical for terrestrially derived organic matter (Fig. 6A). It appears that above a background value of $0.2 \%$ TOC more marine-derived organic matter is incorporated in the sediment, while the background value is accounted for by Type III (vitrinitic) or Type IV (residual, inertinitic) organic material. Indeed, samples with TOC concentrations above $0.2 \%$ plot in the Type II (marine) field of the $T_{\max }$ / HI diagram (Fig. 6A). Supporting evidence for the marine origin of the Tortonian organic matter is presented in the section on lipid analyses below.

Even though the Tortonian sediments are the oldest strata drilled, and even though they may have experienced thermal heating during rifting, it appears that heat flow at Site 654 was low and that almost all Tortonian samples are thermally immature. 
Table 1. Results of whole-rock pyrolysis for samples of Hole 652A.

\begin{tabular}{|c|c|c|c|c|c|c|c|c|c|c|}
\hline $\begin{array}{l}\text { Core, section, } \\
\text { interval }(\mathrm{cm})\end{array}$ & $\begin{array}{l}\text { Depth } \\
\text { (mbsf) }\end{array}$ & $\mathrm{T}_{\max }$ & $s_{1}$ & $\mathrm{~S}_{2}$ & $\mathrm{~S}_{3}$ & PI & $\mathrm{S}_{2} / \mathrm{S}_{3}$ & TOC & HI & $\mathrm{OI}$ \\
\hline \multicolumn{11}{|c|}{ Pleistocene samples of Hole 652A: } \\
\hline $1 \mathrm{R}-1,0-2$ & 0.0 & 511 & 0.03 & 0.20 & 3.57 & 0.14 & 0.05 & 0.18 & 111 & 1983 \\
\hline $1 R-2,51-52$ & 2.0 & 369 & 0.01 & 0.04 & 2.63 & 0.25 & 0.01 & 0.16 & 25 & 1643 \\
\hline $2 \mathrm{R}-3,30-31$ & 6.9 & 422 & 0.22 & 0.11 & 2.73 & 0.69 & 0.04 & 0.18 & 78 & 1950 \\
\hline $2 \mathrm{R}-3,100-101$ & 7.6 & 454 & 0.02 & 0.03 & 3.11 & 0.50 & 0.00 & 1.83 & 1 & 1169 \\
\hline $3 R-1,83-84$ & 17.8 & 304 & 0.01 & 0.06 & 2.92 & 0.17 & 0.02 & 0.06 & & \\
\hline $3 R-1,118-119$ & 18.2 & 464 & 0.01 & 0.08 & 2.84 & 0.12 & 0.02 & 0.04 & & \\
\hline $3 R-2,0-1$ & 18.5 & 416 & 0.76 & 8.03 & 3.76 & 0.09 & 2.13 & 2.69 & 199 & 140 \\
\hline $4 R-4,80-81$ & 32.0 & 382 & 0.01 & 0.03 & 2.71 & 0.25 & 0.01 & 0.07 & & \\
\hline $4 R-5,40-43$ & 33.1 & 286 & 0.00 & 0.01 & 2.77 & & 0.00 & 0.05 & & \\
\hline $4 \mathrm{R}-6,17-20$ & 34.4 & 414 & 0.28 & 3.64 & 3.30 & 0.07 & 1.10 & 1.79 & 203 & 184 \\
\hline $4 R-6,22-24$ & 34.5 & 414 & 0.19 & 2.20 & 3.36 & 0.08 & 0.65 & 1.71 & 128 & 196 \\
\hline $5 \mathrm{R}-1,27-28$ & 36.7 & 455 & 0.01 & 0.11 & 4.19 & 0.08 & 0.02 & 0.02 & & \\
\hline $6 \mathrm{R}-1,33-35$ & 46.1 & 413 & 0.00 & 0.04 & 3.03 & 0.00 & 0.01 & 0.22 & 18 & 1377 \\
\hline $6 \mathrm{R}-1,40-42$ & 46.2 & 454 & 1.24 & 17.10 & 4.63 & 0.07 & 3.69 & 5.82 & 293 & 79 \\
\hline $6 \mathrm{R}-1,53-55$ & 46.3 & 261 & 0.00 & 0.01 & 2.49 & & 0.00 & 0.75 & 1 & 332 \\
\hline $6 \mathrm{R}-1,53-56$ & 46.4 & 262 & 0.00 & 0.00 & 2.33 & & 0.00 & 0.68 & & 342 \\
\hline $6 \mathrm{R}-1,111-114$ & 46.9 & 303 & 0.00 & 0.00 & 2.67 & & 0.00 & 0.33 & & 809 \\
\hline $7 \mathrm{R}-1,21-22$ & 55.6 & 270 & 0.00 & 0.01 & 2.76 & & 0.00 & 0.21 & 4 & 1314 \\
\hline $7 \mathrm{R}-3,1-2$ & 58.4 & 263 & 0.00 & 0.00 & 2.33 & & 0.00 & 0.23 & & 1013 \\
\hline 7R-3, 69-70 & 59.1 & 263 & 0.00 & 0.00 & 2.57 & & 0.00 & 0.22 & & 1168 \\
\hline $7 \mathrm{R}-3,124-127$ & 59.6 & 253 & 0.00 & 0.00 & 2.63 & & 0.00 & 0.20 & & 1315 \\
\hline $8 R-1,56-57$ & 65.5 & 254 & 0.00 & 0.00 & 2.46 & & 0.00 & 0.20 & & 1230 \\
\hline $8 R-1,61-65$ & 65.5 & 254 & 0.00 & 0.00 & 2.57 & & 0.00 & 0.16 & & 1606 \\
\hline \multicolumn{11}{|l|}{ Statistics } \\
\hline Samples & & 23 & 23 & 23 & 23 & & & 23 & 11 & 18 \\
\hline Mean & & 354 & 0.12 & 1.38 & 2.97 & & & 0.77 & 106 & 936 \\
\hline Standard deviation & & 87 & 0.30 & 3.88 & 0.59 & & & 1.32 & 114 & 664 \\
\hline Median & & 369 & 0.01 & 0.03 & 2.76 & & & 0.21 & 78 & 1091 \\
\hline \multicolumn{11}{|c|}{ Pliocene samples of Hole $652 \mathrm{~A}$ : } \\
\hline $9 \mathrm{R}-1,80-83$ & 75.3 & 261 & 0.00 & 0.00 & 2.50 & & 0.00 & 0.23 & & 1086 \\
\hline $10 \mathrm{R}-1,100-103$ & 88.2 & 245 & 0.00 & 0.00 & 2.71 & & 0.00 & 0.18 & & 1505 \\
\hline $10 \mathrm{R}-4,81-82$ & 89.5 & 256 & 0.00 & 0.03 & 2.39 & 0.00 & 0.01 & 0.14 & 21 & 1707 \\
\hline $10 \mathrm{R}-6,140-143$ & 93.1 & 226 & 0.00 & 0.00 & 1.95 & & 0.00 & 0.12 & & 1625 \\
\hline $11 \mathrm{R}-3,98-99$ & 97.8 & 257 & 0.00 & 0.00 & 2.25 & & 0.00 & 0.10 & & 2250 \\
\hline $11 \mathrm{R}-5,130-130$ & 101.1 & 247 & 0.00 & 0.00 & 2.25 & & 0.00 & 0.10 & & 2250 \\
\hline $12 \mathrm{R}-2,78-79$ & 105.3 & 292 & 0.00 & 0.00 & 2.33 & 0.00 & 0.00 & 0.12 & & 1941 \\
\hline $12 \mathrm{R}-5,105-108$ & 110.1 & 255 & 0.00 & 0.00 & 2.29 & & 0.00 & 0.09 & & \\
\hline $13 \mathrm{R}-1,61-62$ & 113.3 & 240 & 0.00 & 0.00 & 2.26 & & 0.00 & 0.10 & & 2260 \\
\hline $13 \mathrm{R}-3,80-81$ & 116.5 & 255 & 0.00 & 0.02 & 1.94 & 0.00 & 0.00 & 0.17 & 11 & 1141 \\
\hline $13 R-3,119-120$ & 116.9 & 423 & 0.00 & 0.00 & 1.75 & & 0.00 & 0.08 & & \\
\hline $13 \mathrm{R}-4,20-23$ & 117.4 & 279 & 0.00 & 0.00 & 1.75 & & 0.00 & 0.04 & & \\
\hline $13 R-4,52-53$ & 117.4 & 217 & 0.00 & 0.00 & 1.94 & & 0.00 & 0.04 & & \\
\hline $14 \mathrm{R}-1,62-63$ & 122.9 & 265 & 0.00 & 0.00 & 2.38 & & 0.00 & $0.0:$ & & \\
\hline $14 \mathrm{R}-2,71-73$ & 124.5 & 217 & 0.00 & 0.00 & 1.78 & & 0.00 & 0.11 & & 1790 \\
\hline $14 \mathrm{R}-3,94-95$ & 126.2 & 325 & 0.01 & 0.07 & 1.97 & & 0.00 & 0.03 & & \\
\hline $14 R-4,66-67$ & 127.4 & 297 & 0.00 & 0.00 & 1.88 & 0.12 & 0.03 & 0.03 & & \\
\hline $15 \mathrm{R}-1,134-137$ & 133.2 & 297 & 0.00 & 0.00 & 2.25 & & 0.00 & 0.03 & & \\
\hline $15 \mathrm{R}-2,19-20$ & 133.6 & 301 & 0.00 & 0.00 & 2.38 & & 0.00 & 0.03 & & \\
\hline $15 \mathrm{R}-4,18-19$ & 136.6 & 324 & 0.02 & 0.10 & 2.44 & & 0.00 & 0.18 & 55 & 1094 \\
\hline $15 R-4,39-40$ & 136.8 & 405 & 0.02 & 0.27 & 1.97 & 0.17 & 0.05 & 0.47 & 57 & 436 \\
\hline $15 \mathrm{R}-5,99-100$ & 138.9 & 282 & 0.01 & 0.08 & 2.05 & 0.07 & 0.13 & 0.02 & & \\
\hline $16 \mathrm{R}-2,82-83$ & 143.8 & 304 & 0.00 & 0.01 & 4.22 & 0.12 & 0.01 & 0.04 & & \\
\hline $16 \mathrm{R}-3,70-73$ & 145.2 & 262 & 0.00 & 0.00 & 1.97 & & 0.00 & 0.02 & & \\
\hline $16 \mathrm{R}-4,1-2$ & 146.0 & 231 & 0.00 & 0.00 & 2.15 & & 0.00 & 0.12 & & 1575 \\
\hline $16 R-4,73-74$ & 146.8 & 254 & 0.00 & 0.01 & 1.89 & & 0.00 & 0.03 & & \\
\hline $16 \mathrm{R}-5,44-45$ & 147.9 & 276 & 0.00 & 0.00 & 2.32 & & 0.00 & 0.06 & & \\
\hline $17 \mathrm{R}-2,68-69$ & 153.4 & 304 & 0.00 & 0.03 & 1.99 & 0.00 & 0.01 & 0.22 & 13 & 904 \\
\hline $17 \mathrm{R}-5,113-114$ & 158.3 & 278 & 0.00 & 0.03 & 1.91 & 0.00 & 0.01 & 0.10 & 30 & 1910 \\
\hline $18 \mathrm{R}-2,46-47$ & 162.8 & 279 & 0.01 & 0.03 & 2.23 & 0.25 & 0.01 & 0.02 & & \\
\hline $18 \mathrm{R}-2,80-81$ & 163.2 & 304 & 0.00 & 0.05 & 1.93 & 0.00 & 0.02 & 0.17 & 29 & 1135 \\
\hline $18 \mathrm{R}-3,81-82$ & 164.6 & 265 & 0.00 & 0.01 & 2.26 & & 0.00 & 0.09 & & \\
\hline $18 \mathrm{R}-4,93-96$ & 166.2 & 259 & 0.00 & 0.00 & 1.74 & 0.00 & 0.02 & & & \\
\hline 18R-6, 93-96 & 169.2 & 446 & 0.00 & 0.01 & 1.74 & & 0.00 & 0.02 & & \\
\hline $18 \mathrm{R}-5,85-86$ & 167.7 & 490 & 0.01 & 0.11 & 2.03 & 0.08 & 0.05 & 0.12 & 91 & 1691 \\
\hline $19 \mathrm{R}-1,47-48$ & 171.0 & 325 & 0.00 & 0.00 & 0.00 & & & 1.75 & & \\
\hline 19R-2, 55-58 & 172.6 & 415 & 0.01 & 0.09 & 2.62 & 0.10 & 0.03 & 0.16 & 56 & 1637 \\
\hline $19 R-4,98-99$ & 176.0 & 437 & 0.01 & 0.09 & 2.77 & 0.10 & 0.03 & 0.17 & 52 & 1629 \\
\hline $19 \mathrm{R}-5,61-62$ & 177.1 & 325 & 0.00 & 0.00 & 0.00 & & & 1.94 & & \\
\hline $19 \mathrm{R}-6,137-140$ & 179.4 & 335 & 0.00 & 0.03 & 2.21 & 0.00 & 0.01 & 0.26 & 1 & 1850 \\
\hline $20 \mathrm{R}-1,71-74$ & 180.9 & 549 & 0.02 & 0.13 & 3.14 & 0.14 & 0.04 & 0.26 & 50 & 1207 \\
\hline
\end{tabular}


Table 1 (continued).

\begin{tabular}{|c|c|c|c|c|c|c|c|c|c|c|}
\hline $\begin{array}{l}\text { Core, section, } \\
\text { interval }(\mathrm{cm})\end{array}$ & $\begin{array}{l}\text { Depth } \\
\text { (mbsf) }\end{array}$ & $\mathrm{T}_{\max }$ & $s_{1}$ & $\mathrm{~S}_{2}$ & $\mathrm{~S}_{3}$ & PI & $\mathrm{S}_{2} / \mathrm{S}_{3}$ & TOC & HI & OI \\
\hline \multicolumn{11}{|c|}{ Pliocene samples of Hole $652 \mathrm{~A}$ (Cont.): } \\
\hline $20 \mathrm{R}-1,100-101$ & 181.2 & 318 & 0.00 & 0.21 & 2.76 & 0.00 & 0.07 & 0.18 & 116 & 1533 \\
\hline $20 \mathrm{R}-5,135-138$ & 187.5 & 305 & 0.00 & 0.00 & 0.00 & & & 2.20 & & \\
\hline $20 \mathrm{R}-6,129-130$ & 189.0 & 397 & 0.00 & 0.30 & 1.25 & 0.00 & 0.24 & 0.29 & 103 & 431 \\
\hline \multicolumn{11}{|l|}{ Statistics } \\
\hline Samples & & 44 & 44 & 44 & 44 & & & 44 & 14 & 23 \\
\hline Mean & & 307 & 0.00 & 0.04 & 2.05 & & & 0.24 & 50 & 1460 \\
\hline Standard deviation & & 75 & 0.01 & 0.07 & 0.73 & & & 0.48 & 34 & 522 \\
\hline Median & & 287 & 0.00 & 0.00 & 2.10 & & & 0.11 & 51 & 1575 \\
\hline
\end{tabular}

Miocene (Messinian) sediments of Hole 652A:

\begin{tabular}{|c|c|c|c|c|c|c|c|c|c|c|}
\hline $21 \mathrm{R}-1,100-101$ & 199.8 & 304 & 0.00 & 0.00 & 0.00 & & & 1.12 & & \\
\hline $21 \mathrm{R}-2,15-18$ & 200.4 & 369 & 0.00 & 0.02 & 0.40 & 0.00 & 0.05 & 0.09 & & \\
\hline $21 R-2,134-137$ & 201.6 & 233 & 0.00 & 0.00 & 0.00 & & & 1.19 & & \\
\hline $22 \mathrm{R}-2,25-28$ & 201.5 & 425 & 0.17 & 0.19 & 1.24 & 0.47 & 0.15 & 0.25 & 76 & 496 \\
\hline $23 \mathrm{R}-1,100-101$ & 219.1 & 326 & 0.00 & 0.00 & 0.00 & & & 0.90 & & \\
\hline $23 \mathrm{R}-2,27-29$ & 219.9 & 466 & 0.02 & 0.17 & 1.15 & 0.11 & 0.14 & 0.23 & 73 & 500 \\
\hline $24 \mathrm{R}-2,83-84$ & 221.0 & 390 & 0.00 & 0.08 & 0.61 & 0.00 & 0.13 & 0.17 & 47 & 359 \\
\hline $24 \mathrm{R}-2,93-94$ & 221.1 & 336 & 0.00 & 0.12 & 1.25 & 0.00 & 0.09 & 0.21 & 57 & 595 \\
\hline $24 R-4,9-10$ & 223.3 & 401 & 0.01 & 0.15 & 0.90 & 0.06 & 0.16 & 0.18 & 83 & 500 \\
\hline $24 \mathrm{R}-4,77-80$ & 224.0 & 305 & 0.00 & 0.11 & 1.02 & 0.00 & 0.10 & 0.14 & 78 & 728 \\
\hline $24 \mathrm{R}-4,112-113$ & 224.3 & 566 & 0.00 & 0.02 & 0.60 & 0.00 & 0.03 & 0.03 & & \\
\hline $25 \mathrm{R}-1,40-41$ & 228.8 & 416 & 0.02 & 0.31 & 1.62 & 0.06 & 0.19 & 0.16 & 193 & 1012 \\
\hline $25 \mathrm{R}-1,61-62$ & 229.6 & 486 & 0.00 & 0.13 & 0.75 & 0.00 & 0.17 & 0.04 & & \\
\hline $25 \mathrm{R}-1,72-73$ & 229.1 & 367 & 0.00 & 0.11 & 0.57 & 0.00 & 0.19 & 0.03 & & \\
\hline $25 \mathrm{R}-2,14-18$ & 230.0 & 441 & 0.00 & 0.16 & 1.07 & 0.00 & 0.14 & 0.12 & 133 & 891 \\
\hline $26 \mathrm{R}-2,40-41$ & 240.0 & 439 & 0.00 & 0.16 & 1.14 & 0.00 & 0.14 & 0.18 & 88 & 633 \\
\hline $26 \mathrm{R}-2,89-90$ & 240.5 & 381 & 0.01 & 0.11 & 0.37 & 0.08 & 0.29 & 0.14 & 78 & 264 \\
\hline $26 \mathrm{R}-2,113-117$ & 240.7 & 432 & 0.00 & 0.13 & 1.36 & 0.00 & 0.09 & 0.15 & 86 & 906 \\
\hline 26R-CC, $5-6$ & 247.5 & 390 & 0.00 & 0.12 & 0.38 & 0.00 & 0.31 & 0.06 & & \\
\hline $27 \mathrm{R}-1,111-112$ & 248.8 & 449 & 0.00 & 0.10 & 1.34 & 0.00 & 0.07 & 0.07 & & \\
\hline $27 \mathrm{R}-3,1-2$ & 250.7 & 443 & 0.00 & 0.09 & 0.81 & 0.00 & 0.11 & 0.10 & 90 & 810 \\
\hline $27 \mathrm{R}-3,10-13$ & 250.8 & 438 & 0.00 & 0.10 & 1.06 & 0.00 & 0.09 & 0.08 & & \\
\hline 27R-CC, 9-11 & 252.4 & 426 & 0.00 & 0.09 & 0.84 & 0.00 & 0.10 & 0.14 & 64 & 600 \\
\hline $28 \mathrm{R}-2,7-10$ & 259.0 & 510 & 0.00 & 0.09 & 0.55 & 0.00 & 0.16 & 0.04 & & \\
\hline $28 \mathrm{R}-2,99-100$ & 259.9 & 480 & 0.00 & 0.12 & 1.23 & 0.00 & 0.09 & 0.08 & & \\
\hline $28 \mathrm{R}-4,28-31$ & 262.2 & 382 & 0.00 & 0.13 & 1.02 & 0.00 & 0.12 & 0.10 & 130 & 1020 \\
\hline $29 \mathrm{R}-1,121-122$ & 268.3 & 396 & 0.01 & 0.13 & 0.89 & 0.07 & 0.14 & 0.09 & & \\
\hline 30R-1, 8-11 & 276.8 & 374 & 0.00 & 0.13 & 0.48 & 0.00 & 0.27 & 0.11 & 118 & 436 \\
\hline $30 \mathrm{R}-3,55-56$ & 280.4 & 434 & 0.02 & 0.22 & 0.84 & 0.08 & 0.26 & 0.18 & 122 & 466 \\
\hline $31 R-1,143-146$ & 287.7 & 530 & 0.03 & 0.22 & 0.57 & 0.12 & 0.39 & 0.07 & & \\
\hline $31 \mathrm{R}-3,89-91$ & 290.2 & 304 & 0.00 & 0.00 & 0.00 & & & 0.05 & & \\
\hline $32 R-1,66-67$ & 296.6 & 471 & 0.01 & 0.16 & 1.70 & 0.06 & 0.09 & 0.10 & 160 & 1700 \\
\hline 32R-1, 97-98 & 296.9 & 401 & 0.00 & 0.13 & 1.03 & 0.00 & 0.12 & 0.09 & & \\
\hline $32 \mathrm{R}-1,98-99$ & 296.9 & 372 & 0.01 & 0.18 & 0.46 & 0.06 & 0.39 & 0.07 & & \\
\hline $32 \mathrm{R}-1,112-113$ & 297.0 & 385 & 0.03 & 0.16 & 0.23 & 0.17 & 0.69 & 0.02 & & \\
\hline $32 R-2,40-41$ & 297.4 & 457 & 0.00 & 0.14 & 0.81 & 0.00 & 0.17 & 0.08 & & \\
\hline $32 \mathrm{R}-2,70-72$ & 297.7 & 429 & 0.01 & 0.15 & 0.35 & 0.06 & 0.42 & 0.03 & & \\
\hline $32 \mathrm{R}-2,125-127$ & 298.3 & 488 & 0.02 & 0.17 & 1.18 & 0.11 & 0.14 & 0.08 & & \\
\hline $33 \mathrm{R}-1,33-34$ & 305.9 & 393 & 0.00 & 0.10 & 0.00 & 0.00 & & 0.09 & & \\
\hline $33 R-3,49-50$ & 309.1 & 463 & 0.01 & 0.15 & 0.23 & 0.06 & 0.65 & 0.05 & & \\
\hline $34 \mathrm{R}-2,50-51$ & 309.9 & 392 & 0.00 & 0.08 & 0.18 & 0.00 & 0.44 & 0.03 & & \\
\hline $34 \mathrm{R}-2,60-61$ & 317.3 & 454 & 0.00 & 0.12 & 1.09 & 0.00 & 0.11 & 0.13 & 92 & 838 \\
\hline $37 \mathrm{R}-1,49-50$ & 344.8 & 409 & 0.02 & 0.31 & 0.74 & 0.06 & 0.41 & 0.17 & 182 & 435 \\
\hline $37 R-2,94-96$ & 346.7 & 373 & 0.01 & 0.12 & 0.13 & 0.08 & 0.92 & 0.03 & & \\
\hline $37 R-6,6-8$ & 351.9 & 448 & 0.03 & 0.28 & 0.91 & 0.10 & 0.30 & 0.19 & 147 & 478 \\
\hline $38 \mathrm{R}-1,21-23$ & 354.2 & 440 & 0.01 & 0.20 & 0.93 & 0.05 & 0.21 & 0.22 & 90 & 422 \\
\hline $38 \mathrm{R}-4,99-100$ & 359.5 & 449 & 0.00 & 0.18 & 1.21 & 0.00 & 0.14 & 0.22 & 81 & 550 \\
\hline $38 R-5,9-12$ & 360.1 & 454 & 0.02 & 0.25 & 0.78 & 0.08 & 0.32 & 0.17 & 147 & 458 \\
\hline $39 \mathrm{R}-1,28-29$ & 364.0 & 450 & 0.03 & 0.19 & 1.58 & 0.14 & 0.12 & 0.20 & 95 & 790 \\
\hline $39 \mathrm{R}-3,81-82$ & 367.7 & 472 & 0.01 & 0.16 & 0.95 & 0.06 & 0.16 & 0.19 & 84 & 500 \\
\hline $39 \mathrm{R}-2,6-8$ & 365.3 & 302 & 0.00 & 0.18 & 0.97 & 0.00 & 0.18 & 0.20 & 90 & 485 \\
\hline $40 \mathrm{R}-1,72-74$ & 374.1 & 337 & 0.00 & 0.21 & 0.00 & 0.00 & & 0.18 & & 116 \\
\hline $40 \mathrm{R}-4,100-101$ & 378.9 & 306 & 0.00 & 0.27 & 0.70 & 0.00 & 0.38 & 0.20 & 135 & 350 \\
\hline 40R-5, 119-120 & 380.4 & 432 & 0.00 & 0.14 & 0.94 & 0.00 & 0.14 & 0.21 & 66 & 447 \\
\hline $40 \mathrm{R}-7,33-35$ & 382.7 & 321 & 0.00 & 0.14 & 0.97 & 0.00 & 0.14 & 0.19 & 73 & 510 \\
\hline $41 \mathrm{R}-1,100-101$ & 384.0 & 424 & 0.00 & 0.09 & 0.88 & 0.00 & 0.10 & 0.17 & 52 & 517 \\
\hline $41 R-2,50-52$ & 385.0 & 306 & 0.00 & 0.15 & 0.65 & 0.00 & 0.23 & 0.24 & 62 & 270 \\
\hline $41 R-6,30-33$ & 390.8 & 396 & 0.00 & 0.15 & 0.41 & 0.00 & 0.36 & 0.24 & 62 & 170 \\
\hline $42 R-2,27-29$ & 394.5 & 306 & 0.00 & 0.19 & 0.60 & 0.00 & 0.31 & 0.21 & 90 & 285 \\
\hline $42 R-5,144-146$ & 398.6 & 564 & 0.00 & 0.13 & 0.75 & 0.00 & 0.17 & 0.11 & 111 & 681 \\
\hline $42 \mathrm{R}-6,49-40$ & 400.7 & 446 & 0.01 & 0.04 & 0.40 & 0.25 & 0.10 & 0.01 & & \\
\hline $43 R-1,48-52$ & 402.9 & 350 & 0.00 & 0.03 & 0.30 & 0.00 & 0.10 & 0.04 & & \\
\hline $43 R-4,38-40$ & 407.2 & 470 & 0.00 & 0.05 & 0.74 & 0.00 & 0.06 & 0.13 & 38 & 569 \\
\hline $43 R-4,40-41$ & 407.3 & 304 & 0.00 & 0.00 & 0.24 & & 0.00 & 0.02 & & \\
\hline
\end{tabular}


Table 1 (continued).

\begin{tabular}{|c|c|c|c|c|c|c|c|c|c|c|}
\hline $\begin{array}{l}\text { Core, section, } \\
\text { interval }(\mathrm{cm})\end{array}$ & $\begin{array}{l}\text { Depth } \\
\text { (mbsf) }\end{array}$ & $\mathrm{T}_{\max }$ & $s_{1}$ & $\mathrm{~S}_{2}$ & $\mathrm{~S}_{3}$ & PI & $\mathrm{S}_{2} / \mathrm{S}_{3}$ & TOC & HI & OI \\
\hline \multicolumn{11}{|c|}{ Miocene (Messinian) sediments of Hole 652A (Cont.): } \\
\hline $44 \mathrm{R}-2,119-120$ & 414.8 & 448 & 0.00 & 0.04 & 1.17 & 0.00 & 0.03 & 0.10 & 401 & 170 \\
\hline $44 \mathrm{R}-3,93-96$ & 416.0 & 419 & 0.02 & 0.13 & 0.77 & 0.14 & 0.16 & 0.13 & 100 & 592 \\
\hline $44 R-3,93-96$ & 416.0 & 424 & 0.00 & 0.08 & 0.73 & 0.00 & 0.10 & 0.11 & 72 & 663 \\
\hline $45 \mathrm{R}-1,137-139$ & 423.2 & 402 & 0.00 & 0.01 & 0.26 & & 0.03 & 0.02 & & \\
\hline $45 \mathrm{R}-2,62-64$ & 423.9 & 509 & 0.00 & 0.17 & 0.63 & 0.00 & 0.26 & 0.13 & 130 & 484 \\
\hline $46 \mathrm{R}-1,60-62$ & 432.1 & 501 & 0.03 & 0.05 & 0.88 & 0.37 & 0.05 & 0.07 & & \\
\hline $46 \mathrm{R}-3,55-56$ & 435.1 & 509 & 0.00 & 0.04 & 0.79 & 0.00 & 0.05 & 0.07 & & \\
\hline $46 \mathrm{R}-3,100-103$ & 435.6 & 410 & 0.00 & 0.05 & 0.50 & 0.00 & 0.10 & 0.05 & & \\
\hline $47 \mathrm{R}-1,64-66$ & 441.7 & 539 & 0.00 & 0.04 & 0.48 & 0.00 & 0.08 & 0.03 & & \\
\hline 47R-3, 97-98 & 445.1 & 579 & 0.00 & 0.09 & 0.78 & 0.00 & 0.11 & 0.08 & & \\
\hline $47 \mathrm{R}-5,32-34$ & 447.4 & 509 & 0.00 & 0.03 & 0.43 & 0.00 & 0.06 & 0.01 & & \\
\hline $48 \mathrm{R}-1,8-10$ & 450.8 & 512 & 0.00 & 0.06 & 0.39 & 0.00 & 0.15 & 0.06 & & \\
\hline $48 \mathrm{R}-2,37-38$ & 452.6 & 542 & 0.00 & 0.07 & 0.75 & 0.00 & 0.09 & 0.06 & & \\
\hline $48 \mathrm{R}-3,130-133$ & 455.0 & 474 & 0.00 & 0.12 & 0.77 & 0.00 & 0.15 & 0.08 & & \\
\hline $49 \mathrm{R}-1,23-25$ & 462.0 & 539 & 0.00 & 0.28 & 0.83 & 0.00 & 0.33 & 0.10 & 280 & 830 \\
\hline $49 \mathrm{R}-1,109-110$ & 461.4 & 550 & 0.00 & 0.20 & 1.44 & 0.00 & 0.13 & 0.15 & 133 & 960 \\
\hline $49 \mathrm{R}-2,95-97$ & 462.8 & 480 & 0.01 & 0.13 & 0.51 & 0.07 & 0.25 & 0.07 & & \\
\hline $50 \mathrm{R}-1,59-62$ & 470.6 & 444 & 0.01 & 0.47 & 0.53 & 0.02 & 0.88 & 0.12 & 391 & 441 \\
\hline $50 \mathrm{R}-2,47-48$ & 472.0 & 513 & 0.00 & 0.11 & 0.90 & 0.00 & 0.12 & 0.15 & 73 & 600 \\
\hline $50 \mathrm{R}-2,85-86$ & 472.4 & 504 & 0.00 & 0.32 & 0.83 & 0.00 & 0.38 & 0.17 & 188 & 488 \\
\hline 50R-3, 9-11 & 473.1 & 466 & 0.01 & 0.21 & 0.71 & 0.05 & 0.29 & 0.14 & 150 & 507 \\
\hline S1R-1, 13-15 & 479.7 & 435 & 0.00 & 0.07 & 0.61 & 0.00 & 0.11 & 0.11 & 63 & 554 \\
\hline $51 \mathrm{R}-1,79-80$ & 480.4 & 428 & 0.00 & 0.30 & 0.88 & 0.00 & 0.34 & 0.17 & 176 & 517 \\
\hline $51 \mathrm{R}-4,46-47$ & 484.6 & 400 & 0.01 & 0.19 & 1.21 & 0.05 & 0.15 & 0.09 & & \\
\hline $51 \mathrm{R}-5,126-128$ & 486.9 & 445 & 0.00 & 0.12 & 0.71 & 0.00 & 0.16 & 0.05 & & \\
\hline $51 \mathrm{R}-6,44-46$ & 487.5 & 419 & 0.01 & 0.24 & 1.33 & 0.04 & 0.18 & 0.21 & 114 & 633 \\
\hline $52 R-1,41-42$ & 489.6 & 428 & 0.01 & 0.28 & 0.80 & 0.04 & 0.35 & 0.23 & 121 & 347 \\
\hline $52 \mathrm{R}-2,78-80$ & 491.5 & 461 & 0.01 & 0.29 & 0.95 & 0.03 & 0.30 & 0.29 & 100 & 327 \\
\hline $52 \mathrm{R}-3,138-140$ & 493.6 & 440 & 0.00 & 0.11 & 0.60 & 0.00 & 0.18 & 0.11 & 100 & 545 \\
\hline $52 \mathrm{R}-4,83-84$ & 494.5 & 432 & 0.01 & 0.27 & 1.08 & 0.04 & 0.25 & 0.28 & 96 & 385 \\
\hline $53 \mathrm{R}-1,70-71$ & 499.5 & 431 & 0.00 & 0.29 & 0.96 & 0.00 & 0.30 & 0.25 & 116 & 384 \\
\hline $53 \mathrm{R}-3,146-148$ & 503.3 & 436 & 0.00 & 0.26 & 1.19 & 0.00 & 0.21 & 0.29 & 89 & 410 \\
\hline $53 R-4,14-16$ & 503.4 & 433 & 0.00 & 0.19 & 1.46 & 0.00 & 0.13 & 0.25 & 76 & 584 \\
\hline $54 \mathrm{R}-1,39-40$ & 508.9 & 393 & 0.00 & 0.21 & 1.18 & 0.00 & 0.17 & 0.25 & 84 & 472 \\
\hline 54R-1, 96-98 & 509.5 & 399 & 0.00 & 0.24 & 1.15 & 0.00 & 0.20 & 0.30 & 80 & 383 \\
\hline $54 \mathrm{R}-3,119-120$ & 512.7 & 401 & 0.00 & 0.21 & 1.15 & 0.00 & 0.18 & 0.29 & 72 & 396 \\
\hline $54 \mathrm{R}-3,132-135$ & 512.8 & 443 & 0.00 & 0.24 & 1.59 & 0.00 & 0.15 & 0.27 & 88 & 588 \\
\hline $55 \mathrm{R}-1,59-61$ & 518.7 & 526 & 0.00 & 0.19 & 1.30 & 0.00 & 0.14 & 0.20 & 95 & 650 \\
\hline $55 \mathrm{R}-2,60-61$ & 520.2 & 478 & 0.00 & 0.28 & 1.67 & 0.00 & 0.16 & 0.30 & 93 & 556 \\
\hline $55 \mathrm{R}-3,30-32$ & 521.4 & 442 & 0.00 & 0.23 & 0.92 & 0.00 & 0.25 & 0.22 & 104 & 418 \\
\hline $55 \mathrm{R}-5,145-148$ & 525.6 & 465 & 0.00 & 0.20 & 1.13 & 0.00 & 0.17 & 0.23 & 86 & 491 \\
\hline $56 \mathrm{R}-2,32-34$ & 529.6 & 431 & 0.00 & 0.27 & 1.15 & 0.00 & 0.23 & 0.28 & 96 & 410 \\
\hline $56 \mathrm{R}-6,11-13$ & 535.4 & 424 & 0.00 & 0.16 & 1.03 & 0.00 & 0.15 & 0.23 & 69 & 447 \\
\hline $57 \mathrm{R}-3,62-64$ & 541.0 & 394 & 0.00 & 0.13 & 1.16 & 0.00 & 0.11 & 0.19 & 68 & 610 \\
\hline $57 \mathrm{R}-3,89-90$ & 541.3 & 400 & 0.00 & 0.19 & 1.27 & 0.00 & 0.14 & 0.27 & 70 & 470 \\
\hline 57R-3, 119-120 & 541.6 & 388 & 0.00 & 0.13 & 0.63 & 0.00 & 0.20 & 0.24 & 54 & 262 \\
\hline $58 \mathrm{R}-1,21-22$ & 546.8 & 448 & 0.01 & 0.27 & 0.82 & 0.04 & 0.32 & 0.27 & 100 & 303 \\
\hline $58 \mathrm{R}-1,48-50$ & 547.1 & 425 & 0.05 & 0.44 & 0.28 & 0.10 & 1.57 & 0.28 & 157 & 100 \\
\hline $58 \mathrm{R}-6,30-32$ & 554.4 & 456 & 0.02 & 0.28 & 1.08 & 0.07 & 0.25 & 0.26 & 107 & 415 \\
\hline $59 \mathrm{R}-1,7-9$ & 556.4 & 494 & 0.00 & 0.48 & 0.28 & 0.00 & 1.71 & 0.33 & 145 & 84 \\
\hline $59 \mathrm{R}-2,139-140$ & 559.1 & 330 & 0.02 & 0.55 & 0.52 & 0.04 & 1.05 & 0.32 & 171 & 162 \\
\hline $59 \mathrm{R}-2,140-150$ & 559.2 & 383 & 0.02 & 0.12 & 0.72 & 0.14 & 0.16 & 0.20 & 60 & 360 \\
\hline $59 \mathrm{R}-4,18-20$ & 561.0 & 386 & 0.00 & 0.15 & 0.87 & 0.00 & 0.17 & 0.26 & 57 & 334 \\
\hline $60 \mathrm{R}-1,16-18$ & 566.2 & 387 & 0.00 & 0.12 & 0.83 & 0.00 & 0.14 & 0.28 & 42 & 296 \\
\hline $60 \mathrm{R}-1,87-88$ & 566.9 & 436 & 0.00 & 0.62 & 1.38 & 0.00 & 0.44 & 0.30 & 206 & 460 \\
\hline $60 \mathrm{R}-2,2-4$ & 567.6 & 432 & 0.00 & 0.21 & 0.85 & 0.00 & 0.24 & 0.33 & 63 & 257 \\
\hline $60 \mathrm{R}-3,100-101$ & 570.0 & 398 & 0.00 & 0.10 & 0.91 & 0.00 & 0.10 & 0.29 & 34 & 313 \\
\hline $60 \mathrm{R}-5,106-108$ & 573.1 & 384 & 0.00 & 0.16 & 2.14 & 0.00 & 0.07 & 0.28 & 57 & 764 \\
\hline 61R-2, 106-107 & 578.2 & 524 & 0.09 & 0.17 & 0.19 & 0.35 & 0.89 & 0.11 & 154 & 172 \\
\hline $61 \mathrm{R}-4,146-147$ & 581.6 & 440 & 0.01 & 0.53 & 0.65 & 0.02 & 0.81 & 0.38 & 139 & 171 \\
\hline $61 \mathrm{R}-5,90-91$ & 582.5 & 451 & 0.01 & 0.49 & 0.59 & 0.02 & 0.83 & 0.42 & 116 & 140 \\
\hline $62 \mathrm{R}-3,91-93$ & 589.1 & 467 & 0.00 & 0.36 & 0.68 & 0.00 & 0.52 & 0.39 & 92 & 174 \\
\hline $62 R-4,112-114$ & 590.8 & 447 & 0.00 & 0.51 & 0.45 & 0.00 & 1.13 & 0.37 & 137 & 121 \\
\hline $63 R-3,40-42$ & 598.3 & 453 & 0.27 & 7.49 & 0.11 & 0.03 & 68.09 & 2.05 & 365 & 5 \\
\hline $63 R-2,139-140$ & 597.8 & 448 & 0.01 & 0.13 & 1.26 & 0.07 & 0.10 & 0.36 & 36 & 350 \\
\hline $64 \mathrm{R}-1,23-24$ & 604.7 & 450 & 0.02 & 0.34 & 0.65 & 0.06 & 0.52 & 0.50 & 68 & 130 \\
\hline $64 \mathrm{R}-1,92-94$ & 605.4 & 454 & 4.72 & 64.58 & 0.86 & 0.07 & 75.09 & 10.52 & 613 & 8 \\
\hline $64 R-1,103-104$ & 605.5 & 457 & 5.94 & 77.15 & 0.73 & 0.07 & 105.6 & 6.92 & 738 & 6 \\
\hline $64 \mathrm{R}-1,105-107$ & 605.6 & 455 & 3.63 & 56.57 & 0.89 & 0.06 & 63.56 & 9.39 & 602 & 9 \\
\hline $64 R-1,104-111$ & 605.7 & 457 & 3.32 & 74.26 & 0.53 & 0.04 & 140.11 & 8.43 & 880 & 6 \\
\hline $64 \mathrm{R}-3,22-23$ & 607.7 & 450 & 0.18 & 0.10 & 0.90 & 0.64 & 0.11 & 0.32 & 31 & 281 \\
\hline $64 R-3,59-61$ & 608.1 & 359 & 0.00 & 0.02 & 0.73 & 0.00 & 0.02 & 0.23 & 8 & 317 \\
\hline $64 R-1,104-111$ & 605.6 & 446 & 2.66 & 19.73 & 0.65 & 0.12 & 30.35 & 2.28 & 865 & 28 \\
\hline $64 \mathrm{R}-3,0-1$ & 607.5 & 527 & 0.00 & 0.54 & 0.79 & 0.00 & 0.68 & 0.53 & 101 & 149 \\
\hline $65 R-2,55-56$ & 616.3 & 511 & 0.00 & 0.14 & 1.07 & 0.00 & 0.13 & 0.36 & 38 & 297 \\
\hline
\end{tabular}


Table 1 (continued).

\begin{tabular}{|c|c|c|c|c|c|c|c|c|c|c|}
\hline $\begin{array}{l}\text { Core, section, } \\
\text { interval }(\mathrm{cm})\end{array}$ & $\begin{array}{l}\text { Depth } \\
\text { (mbsf) }\end{array}$ & $\mathrm{T}_{\max }$ & $\mathrm{s}_{1}$ & $\mathrm{~S}_{2}$ & $\mathrm{~S}_{3}$ & PI & $\mathrm{S}_{2} / \mathrm{S}_{3}$ & TOC & HI & OI \\
\hline \multicolumn{11}{|c|}{ Miocene (Messinian) sediments of Hole $652 \mathrm{~A}$ (Cont.): } \\
\hline $65 \mathrm{R}-2,77-79$ & 616.5 & 448 & 0.00 & 0.20 & 1.07 & 0.00 & 0.18 & 0.63 & 31 & 169 \\
\hline $65 R-2,91-92$ & 616.6 & 527 & 0.00 & 0.21 & 0.72 & 0.00 & 0.29 & 0.38 & 55 & 189 \\
\hline $65 \mathrm{R}-2,109-111$ & 616.8 & 455 & 0.00 & 0.11 & 0.83 & 0.00 & 0.13 & 0.37 & 29 & 224 \\
\hline $65 R-4,2-4$ & 618.7 & 351 & 0.00 & 0.00 & 0.74 & & 0.00 & 0.16 & & 462 \\
\hline $65 R-4,2-4$ & 618.7 & 579 & 0.00 & 0.26 & 0.63 & 0.00 & 0.41 & 0.39 & 66 & 161 \\
\hline $65 R-6,105-107$ & 622.8 & 481 & 0.00 & 0.32 & 0.57 & 0.00 & 0.56 & 0.33 & 96 & 172 \\
\hline $66 \mathrm{R}-1,90-92$ & 625.4 & 447 & 0.00 & 0.06 & 0.43 & 0.00 & 0.13 & 0.22 & 27 & 195 \\
\hline $66 \mathrm{R}-3,24-25$ & 627.7 & 407 & 0.00 & 0.07 & 0.75 & 0.00 & 0.09 & 0.33 & 21 & 227 \\
\hline $66 \mathrm{R}-3,74-76$ & 628.2 & 449 & 0.01 & 0.11 & 0.67 & 0.08 & 0.16 & 0.33 & 33 & 203 \\
\hline $66 \mathrm{R}-3,98-99$ & 628.4 & 529 & 0.00 & 0.26 & 0.67 & 0.00 & 0.38 & 0.33 & 78 & 203 \\
\hline $66 \mathrm{R}-3,119-120$ & 628.7 & 514 & 0.00 & 0.16 & 0.82 & 0.00 & 0.19 & 0.34 & 47 & 241 \\
\hline $67 \mathrm{R}-2,77-78$ & 636.5 & 392 & 0.00 & 0.06 & 0.67 & 0.00 & 0.18 & 0.30 & 20 & 223 \\
\hline $67 R-5,144-146$ & 641.6 & 426 & 0.00 & 0.08 & 0.69 & 0.00 & 0.11 & 0.31 & 25 & 222 \\
\hline $68 \mathrm{R}-1,24-25$ & 644.0 & 528 & 0.00 & 0.11 & 0.76 & 0.00 & 0.14 & 0.22 & 50 & 345 \\
\hline $68 \mathrm{R}-1,146-148$ & 645.3 & 348 & 0.00 & 0.01 & 0.41 & & 0.02 & 0.28 & 3 & 146 \\
\hline $68 R-6,51-53$ & 651.8 & 388 & 0.00 & 0.05 & 0.64 & 0.00 & 0.07 & 0.30 & 16 & 213 \\
\hline $69 \mathrm{R}-1,2-4$ & 653.4 & 486 & 0.00 & 0.09 & 0.63 & 0.00 & 0.14 & 0.28 & 32 & 225 \\
\hline $69 R-3,47-48$ & 656.9 & 356 & 0.00 & 0.01 & 0.68 & 0.01 & 0.26 & 3 & 261 & \\
\hline $69 R-4,70-72$ & 658.6 & 455 & 0.00 & 0.09 & 0.69 & 0.00 & 0.13 & 0.27 & 33 & 255 \\
\hline $70 \mathrm{R}-2,64-66$ & 655.2 & 453 & 0.00 & 0.03 & 0.62 & 0.00 & 0.04 & 0.25 & 12 & 248 \\
\hline $70 \mathrm{R}-3,119-120$ & 667.3 & 497 & 0.00 & 0.18 & 0.60 & 0.00 & 0.30 & 0.31 & 58 & 193 \\
\hline $70 \mathrm{R}-4,24-26$ & 667.8 & 478 & 0.04 & 0.31 & 0.64 & 0.12 & 0.48 & 0.27 & 114 & 237 \\
\hline $71 \mathrm{R}-1,78-80$ & 673.6 & 306 & 0.00 & 0.26 & 0.71 & 0.00 & 0.36 & 0.24 & 108 & 295 \\
\hline $71 \mathrm{R}-2,45-47$ & 674.8 & 458 & 0.02 & 0.25 & 0.71 & 0.08 & 0.35 & 0.31 & 80 & 229 \\
\hline $72 \mathrm{R}-1,24-25$ & 682.7 & 456 & 0.02 & 0.21 & 0.52 & 0.09 & 0.40 & 0.25 & 84 & 208 \\
\hline $72 \mathrm{R}-1,54-56$ & 683.0 & 311 & 0.00 & 0.13 & 0.00 & 0.00 & & 0.08 & & \\
\hline $72 \mathrm{R}-2,50-52$ & 684.4 & 306 & 0.03 & 0.00 & 0.00 & 0.00 & & 0.09 & & \\
\hline $72 \mathrm{R}-2,80-82$ & 684.9 & 401 & 0.03 & 0.00 & 0.05 & 0.05 & & 0.07 & & \\
\hline $73 R-1,70-71$ & 692.9 & 402 & 0.08 & 0.05 & 0.00 & 0.00 & 1.60 & 0.12 & 66 & 41 \\
\hline $73 \mathrm{R}-3,99-101$ & 696.2 & 393 & 0.16 & 0.00 & 0.00 & 0.00 & & 0.20 & 80 & 0 \\
\hline $74 R-1,4-6$ & 701.9 & 388 & 0.10 & 0.60 & 0.17 & 0.17 & 0.16 & 0.02 & & \\
\hline $74 R-2,54-56$ & 703.9 & 306 & 0.00 & 0.00 & 1.00 & 1.00 & & 0.10 & & \\
\hline $74 R-4,0-1$ & 706.4 & 423 & 0.05 & 0.27 & 0.00 & 0.00 & 0.18 & 0.24 & 20 & 112 \\
\hline $74 R-4,97-98$ & 707.4 & 239 & 0.00 & 0.03 & 0.00 & 0.00 & & 0.06 & & \\
\hline $74 R-5,52-54$ & 708.4 & 510 & 0.02 & 0.16 & 0.61 & 0.11 & 0.26 & 0.24 & 66 & 254 \\
\hline $75 R-4,23-25$ & 715.2 & 512 & 0.02 & 0.19 & 0.80 & 0.10 & 0.23 & 0.27 & 70 & 296 \\
\hline $75 \mathrm{R}-6,111-113$ & 719.1 & 305 & 0.00 & 0.06 & 0.47 & 0.00 & 0.12 & 0.16 & 37 & 293 \\
\hline $75 \mathrm{R}-\mathrm{CC}, 0-2$ & 720.0 & 434 & 0.00 & 0.07 & 0.88 & 0.00 & 0.07 & 0.26 & 26 & 338 \\
\hline 75R-CC, $7-9$ & 720.1 & 305 & 0.00 & 0.24 & 0.82 & 0.00 & 0.29 & 0.35 & 68 & 234 \\
\hline \multicolumn{11}{|l|}{ Statistics } \\
\hline San & & 182 & 182 & 182 & 182 & & & 182 & 131 & 130 \\
\hline Mean & & 428 & 0.12 & 1.81 & 0.74 & & & 0.42 & 114 & 402 \\
\hline Standard deviation & & 67 & 0.69 & 10.16 & 0.39 & & & 1.30 & 140 & 258 \\
\hline Median & & 436 & 0.00 & 0.15 & 0.74 & & & 0.20 & 83 & 360 \\
\hline
\end{tabular}

Note. Depth in meters below seafloor; $\mathrm{T}_{\max }$ in ${ }^{\circ} \mathrm{C} ; \mathrm{S}_{1}$ and $\mathrm{S}_{2}$ in $\mathrm{mg}$ hydrocarbons $/ \mathrm{g}$ sediment; $\mathrm{S}_{3}$ in $\mathrm{mg} \mathrm{CO} / \mathrm{g}$ sediment; $\mathrm{PI}$ in $\mathrm{mg}$ free hydrocarbons $/ \mathrm{g}$ sediment; TOC in weight $\%$; $\mathrm{HI}$ in $\mathrm{mg}$ hydrocarbons $/ \mathrm{g}$ TOC; OI in $\mathrm{mg}$ $\mathrm{CO}_{2} / \mathrm{g}$ TOC.

Messinian samples of both Holes 652A and 654A cluster on the boundary between marine and terrestrial organic matter in the $\mathrm{T}_{\max } / \mathrm{HI}$ diagram (Figs. 6B and 7). Terrestrial input is noticeable in particular in Hole $652 \mathrm{~A}$, where the only notable exception are samples from the oil shale interval of Core 107$652 \mathrm{~A}-64 \mathrm{R}$, which contain Type I organic matter and which are an excellent oil source rock due to maturity within the oil window. As will be discussed below, the oil-shale interval actually appears to produce oil now. None of the samples from the Messinian section of Hole 654A that appear to contain mixtures of marine and terrestrial organic matter have reached thermal maturity yet, but they appear to contain relatively more TOC of marine sources when compared to those of Hole 652A. Hypersaline conditions (obvious from the occurrence of evaporites in the section) did not have any enhancing effects on the preservation, because most of the samples from the evaporitic section of Hole $654 \mathrm{~A}$ are organic matter-lean.

TOC in sediments of Pliocene and Pleistocene age at both sites plots in the field of recycled, inert organic matter in the
$\mathrm{T}_{\max } / \mathrm{HI}$ diagram and plot in the Type III field (Figs. 6C and 6D). A few exceptions are the samples that contain higher concentrations of organic matter, which then have the higher HI characteristic of marine origin. For a comparison with immature marine organic matter from sapropels high in marine TOC, samples of sapropels and surrounding background sediment lean in TOC from Core $107-655 \mathrm{~A}-2 \mathrm{H}$ and $-3 \mathrm{H}$ are also plotted in Figure 6D.

In spite of differences between Sites 652 and 654 and between the litho- and chronostratigraphic units of each site, statistical evaluation failed to recognize significant differences between the data (Fig. 7). The parameters $\mathrm{HI}$ and $\mathrm{T}_{\max }$ vary too widely, and ranges overlap considerably. In spite of widely differing depositional environments, pyrolysis results should thus be viewed with caution in interpreting nearshore paleoenvironments. Two points can be made from the summary plots, however: (1) Site 652 must have experienced a more intense thermal heating in the Messinian than Site 654 , as determined by $T_{\max }$ values, and facies patterns and differences in stratigraphic units 
Table 2. Results of whole-rock pyrolysis for samples of Hole 654A.

\begin{tabular}{|c|c|c|c|c|c|c|c|c|c|c|}
\hline $\begin{array}{l}\text { Core, section, } \\
\text { interval }(\mathrm{cm})\end{array}$ & $\begin{array}{l}\text { Depth } \\
\text { (mbsf) }\end{array}$ & $\mathrm{T}_{\max }$ & $\mathrm{S}_{1}$ & $\mathrm{~S}_{2}$ & $\mathrm{~S}_{3}$ & PI & $\mathrm{S}_{2} / \mathrm{S}_{3}$ & TOC & HI & OI \\
\hline \multicolumn{11}{|c|}{ Hole 654A Pleistocene samples: } \\
\hline IR-1, 75-77 & 0.75 & 380 & 0.00 & 0.00 & 2.52 & & 0.00 & 0.04 & & \\
\hline $2 \mathrm{R}-1,73-76$ & 5.33 & 446 & 0.04 & 0.09 & 3.67 & 0.33 & 0.02 & 0.08 & & \\
\hline $2 \mathrm{R}-1,100-101$ & 5.60 & 378 & 0.08 & 0.16 & 2.90 & 0.33 & 0.05 & 0.06 & & \\
\hline $2 \mathrm{R}-3,30-31$ & 7.90 & 309 & 0.02 & 0.02 & 2.95 & 0.50 & 0.00 & 0.05 & & \\
\hline $2 \mathrm{R}-3,64-67$ & 8.24 & 342 & 0.02 & 0.09 & 2.42 & 0.20 & 0.03 & 0.20 & 45 & 1210 \\
\hline $3 R-2,49-51$ & 13.99 & 387 & 0.15 & 0.35 & 3.56 & 0.30 & 0.09 & 0.16 & 218 & 2225 \\
\hline $3 R-5,0-1$ & 18.01 & 384 & 0.07 & 0.09 & 2.82 & 0.44 & 0.03 & 0.04 & & \\
\hline $3 R-5,49-51$ & 18.49 & 309 & 0.04 & 0.09 & 2.75 & 0.33 & 0.03 & 0.02 & & \\
\hline $3 R-6,49-51$ & 19.99 & 380 & 0.07 & 0.15 & 2.52 & 0.32 & 0.05 & 0.05 & & \\
\hline $4 R-1,49-51$ & 21.89 & 375 & 0.11 & 0.12 & 2.64 & 0.50 & 0.04 & 0.04 & & \\
\hline $4 R-4,74-75$ & 26.64 & 307 & 0.02 & 0.02 & 2.42 & 0.50 & 0.00 & 0.04 & & \\
\hline $4 R-5,50-51$ & 27.90 & 380 & 0.10 & 0.16 & 2.32 & 0.38 & 0.06 & 0.08 & & \\
\hline $5 R-1,50-51$ & 31.30 & 381 & 0.02 & 0.02 & 2.33 & 0.50 & 0.00 & 0.03 & & \\
\hline $5 R-2,119-120$ & 33.49 & 309 & 0.00 & 0.03 & 2.73 & 0.00 & 0.01 & 0.04 & & \\
\hline $5 R-3,50-51$ & 34.40 & 309 & 0.03 & 0.02 & 2.42 & 0.75 & 0.00 & 0.06 & & \\
\hline SR-3, 138-139 & 35.18 & 299 & 0.07 & 0.04 & 2.25 & 0.70 & 0.01 & 0.04 & & \\
\hline 5R-CC, $5-7$ & 40.05 & 424 & 0.16 & 1.81 & 3.15 & 0.08 & 0.57 & 0.93 & 194 & 338 \\
\hline $6 \mathrm{R}-1,50-51$ & 40.80 & 419 & 0.02 & 0.08 & 2.38 & 0.20 & 0.03 & 0.08 & & \\
\hline $6 \mathrm{R}-2,50-51$ & 42.30 & 266 & 0.00 & 0.01 & 3.35 & 0.00 & & 0.05 & & \\
\hline $6 R-4,133-135$ & 46.13 & 423 & 0.22 & 2.61 & 4.00 & 0.08 & 0.65 & 1.18 & 221 & 338 \\
\hline $6 \mathrm{R}-4,140-141$ & 46.20 & 413 & 1.01 & 0.37 & 5.54 & 0.09 & 1.87 & 4.09 & 253 & 135 \\
\hline $6 \mathrm{R}-4,142-144$ & 46.22 & 414 & 0.68 & 7.96 & 4.91 & 0.08 & 1.62 & 3.00 & 265 & 163 \\
\hline $6 R-5,15-17$ & 46.45 & 404 & 0.09 & 0.36 & 3.53 & 0.20 & 0.10 & 0.31 & 116 & 1138 \\
\hline $6 \mathrm{R}-5,50-51$ & 46.80 & 284 & 0.05 & 0.04 & 2.56 & 0.62 & 0.01 & 0.11 & 36 & 2327 \\
\hline 7R-CC, 7-8 & 59.07 & 250 & 0.01 & 0.01 & 2.45 & 0.50 & 0.00 & 0.05 & & \\
\hline $8 \mathrm{R}-2,49-50$ & 61.09 & 399 & 0.08 & 0.34 & 2.25 & 0.19 & 0.15 & 0.30 & 113 & 750 \\
\hline $8 R-4,42-43$ & 64.02 & 423 & 0.34 & 4.65 & 3.95 & 0.07 & 1.17 & 1.70 & 273 & 232 \\
\hline \multicolumn{11}{|l|}{ Statistics: } \\
\hline Samples & & 27 & 27 & 27 & 27 & & & 27 & 10 & 10 \\
\hline Mean & & 360 & 0.13 & 1.03 & 2.97 & & & 0.44 & 173 & 886 \\
\hline Standard deviation & & 54 & 0.22 & 2.48 & 0.82 & & & 0.95 & 89 & 828 \\
\hline Median & & 380 & 0.07 & 0.09 & 2.64 & & & 0.06 & 206 & 544 \\
\hline \multicolumn{11}{|c|}{ Hole 654A Pliocene samples: } \\
\hline $9 \mathrm{R}-1,50-51$ & 69.80 & 379 & 0.06 & 0.12 & 2.45 & 0.33 & 0.04 & 0.12 & 100 & 2041 \\
\hline $9 \mathrm{R}-1,60-63$ & 69.90 & 389 & 0.01 & 0.05 & 2.68 & 0.17 & 0.01 & 0.05 & & \\
\hline $9 \mathrm{R}-3,50-51$ & 72.80 & 358 & 0.03 & 0.09 & 2.32 & 0.25 & 0.03 & 0.06 & & \\
\hline $10 \mathrm{R}-1,50-51$ & 79.50 & 350 & 0.03 & 0.07 & 2.62 & 0.30 & 0.02 & 0.04 & & \\
\hline 11R-1, 50-51 & 89.20 & 307 & 0.04 & 0.07 & 2.70 & 0.40 & 0.02 & 0.03 & & \\
\hline $11 \mathrm{R}-1,78-80$ & 89.48 & 309 & 0.00 & 0.02 & 3.37 & 0.00 & 0.00 & 0.04 & & \\
\hline $11 \mathrm{R}-3,78-80$ & 92.48 & 281 & 0.03 & 0.04 & 2.65 & 0.50 & 0.01 & 0.05 & & \\
\hline $14 \mathrm{R}-1,89-90$ & 118.59 & 309 & 0.03 & 0.14 & 2.57 & 0.19 & 0.05 & 0.07 & & \\
\hline $15 \mathrm{R}-4,17-20$ & 132.07 & 352 & 0.00 & 0.04 & 2.64 & 0.00 & 0.01 & 0.09 & & \\
\hline $15 \mathrm{R}-7,50-51$ & 136.90 & 434 & 0.02 & 0.08 & 2.11 & 0.20 & 0.00 & 0.15 & 53 & 1406 \\
\hline $16 \mathrm{R}-6,10-21$ & 144.50 & 302 & 0.02 & 0.10 & 1.89 & 0.17 & 0.05 & 0.05 & & \\
\hline $16 \mathrm{R}-6,24-25$ & 144.64 & 304 & 0.04 & 0.14 & 1.40 & 0.22 & 0.09 & 0.05 & & \\
\hline $17 \mathrm{R}-2,117-120$ & 149.07 & 392 & 0.06 & 0.25 & 1.57 & 0.20 & 0.15 & 0.07 & & \\
\hline $17 \mathrm{R}-4,53-56$ & 151.43 & 301 & 0.00 & 0.02 & 1.61 & 0.00 & 0.01 & 0.03 & & \\
\hline $18 \mathrm{R}-5,146-149$ & 163.56 & 263 & 0.01 & 0.00 & 1.38 & 0.00 & 0.03 & & & \\
\hline $18 \mathrm{R}-7,49-52$ & 165.59 & 304 & 0.00 & 0.02 & 1.56 & 0.00 & 0.01 & 0.06 & & \\
\hline $19 \mathrm{R}-3,23-26$ & 169.03 & 304 & 0.03 & 0.05 & 1.82 & 0.37 & 0.02 & 0.08 & & \\
\hline $19 \mathrm{R}-4,54-57$ & 170.84 & 352 & 0.07 & 0.19 & 1.44 & 0.27 & 0.13 & 0.09 & & \\
\hline $20 \mathrm{R}-4,47-50$ & 179.97 & 247 & 0.01 & 0.01 & 1.48 & 0.50 & 0.00 & 0.06 & & \\
\hline $20 \mathrm{R}-4,119-120$ & 180.69 & 256 & 0.05 & 0.11 & 1.42 & 0.31 & 0.07 & 0.03 & & \\
\hline $20 \mathrm{R}-5,18-21$ & 181.18 & 424 & 0.02 & 0.00 & 0.46 & 1.00 & 0.00 & 0.02 & & \\
\hline $21 \mathrm{R}-3,80-82$ & 188.40 & 303 & 0.02 & 0.10 & 1.41 & 0.17 & 0.07 & 0.02 & & \\
\hline $21 \mathrm{R}-5,20-22$ & 190.80 & 371 & 0.04 & 0.18 & 1.77 & 0.18 & 0.10 & 0.08 & & \\
\hline $23 R-2,73-76$ & 206.03 & 327 & 0.03 & 0.12 & 1.70 & 0.21 & 0.07 & 0.04 & & \\
\hline $23 R-4,73-76$ & 209.03 & 364 & 0.02 & 0.11 & 1.85 & 0.17 & 0.05 & 0.12 & 91 & 1541 \\
\hline $24 \mathrm{R}-5,132-135$ & 220.72 & 366 & 0.00 & 0.03 & 2.00 & 0.00 & 0.01 & 0.11 & 27 & 1818 \\
\hline $24 \mathrm{R}-6,56-59$ & 221.46 & 337 & 0.03 & 0.07 & 2.07 & 0.30 & 0.03 & 0.07 & & \\
\hline $25 \mathrm{R}-5,42-45$ & 229.52 & 304 & 0.04 & 0.19 & 1.85 & 0.18 & 0.10 & 0.08 & & \\
\hline $25 \mathrm{R}-5,135-138$ & 230.45 & 304 & 0.04 & 0.05 & 1.94 & 0.50 & 0.02 & 0.09 & & \\
\hline $26 \mathrm{R}-4,60-64$ & 237.80 & 318 & 0.19 & 0.26 & 3.00 & 0.43 & 0.08 & 0.26 & 100 & 1153 \\
\hline \multicolumn{11}{|l|}{ Statistics } \\
\hline Samples & & 30 & 30 & 30 & 30 & & & 30 & 5 & 5 \\
\hline Mean & & 329 & 0.03 & 0.09 & 1.96 & & & 0.07 & 74 & 1592 \\
\hline Standard deviation & & 46 & 0.04 & 0.07 & 0.61 & & & 0.05 & 33 & 347 \\
\hline Median & & 309 & 0.03 & 0.08 & 1.87 & & & 0.06 & 91 & 1541 \\
\hline
\end{tabular}


Table 2 (continued).

\begin{tabular}{|c|c|c|c|c|c|c|c|c|c|c|}
\hline $\begin{array}{l}\text { Core, section, } \\
\text { interval }(\mathrm{cm})\end{array}$ & $\begin{array}{l}\text { Depth } \\
\text { (mbsf) }\end{array}$ & $\mathrm{T}_{\max }$ & $\mathrm{S}_{1}$ & $\mathrm{~S}_{2}$ & $\mathrm{~S}_{3}$ & PI & $\mathrm{S}_{2} / \mathrm{S}_{3}$ & TOC & HI & OI \\
\hline \multicolumn{11}{|c|}{ Hole 654A Miocene (Messinian) samples: } \\
\hline $28 \mathrm{R}-1,60-64$ & 252.60 & 429 & 0.04 & 0.18 & 0.41 & 0.18 & 0.43 & 0.10 & 180 & 410 \\
\hline $28 \mathrm{R}-3,103-107$ & 256.02 & 304 & 0.14 & 0.14 & 0.94 & 0.50 & 0.14 & 0.02 & & \\
\hline $29 \mathrm{R}-1,145-149$ & 263.15 & 385 & 0.03 & 0.18 & 0.43 & 0.15 & 0.41 & 0.20 & 90 & 215 \\
\hline $29 \mathrm{R}-2,90-93$ & 264.10 & 277 & 0.03 & 0.03 & 1.23 & 0.50 & 0.02 & 0.04 & & \\
\hline $31 \mathrm{R}-1,114-117$ & 277.44 & 401 & 0.04 & 0.25 & 0.69 & 0.14 & 0.36 & 0.27 & 92 & 255 \\
\hline $32 \mathrm{R}-1,90-93$ & 281.80 & 374 & 0.01 & 0.07 & 1.01 & 0.12 & 0.06 & 0.02 & & \\
\hline $34 \mathrm{R}-\mathrm{CC}, 9-10$ & 300.29 & 425 & 0.00 & 0.10 & 1.09 & 0.00 & 0.09 & 0.16 & 62 & 681 \\
\hline $35 \mathrm{R}-1,88-90$ & 301.18 & 380 & 0.01 & 0.07 & 0.65 & 0.12 & 0.10 & 0.09 & & \\
\hline $37 \mathrm{R}-1,75-76$ & 320.35 & 374 & 0.00 & 0.01 & 1.13 & & 0.00 & 0.27 & 3 & 418 \\
\hline $38 \mathrm{R}-2,33-39$ & 331.13 & 359 & 1.68 & 3.32 & 1.05 & 0.34 & 3.16 & 3.21 & 100 & 32 \\
\hline $38 \mathrm{R}-2,140-150$ & 332.20 & 304 & 0.04 & 0.05 & 1.35 & 0.50 & 0.03 & 0.19 & 26 & 710 \\
\hline $38 \mathrm{R}-2,148-149$ & 332.28 & 353 & 1.22 & 3.48 & 2.22 & 0.26 & 1.56 & 2.31 & 150 & 96 \\
\hline $39 \mathrm{R}-1,7-10$ & 338.67 & 398 & 0.54 & 5.54 & 2.19 & 0.09 & 2.52 & 2.60 & 213 & 84 \\
\hline $39 \mathrm{R}-1,102-104$ & 339.62 & 383 & 0.15 & 1.14 & 1.62 & 0.12 & 0.70 & 0.93 & 122 & 174 \\
\hline $40 \mathrm{R}-2,60-63$ & 350.30 & 363 & 0.45 & 1.01 & 1.73 & 0.31 & 0.58 & 0.55 & 183 & 314 \\
\hline $40 \mathrm{R}-3,139-140$ & 352.59 & 272 & 0.00 & 0.03 & 2.40 & 0.00 & 0.01 & 0.39 & 7 & 615 \\
\hline $40 \mathrm{R}-5,65-67$ & 354.85 & 383 & 0.61 & 1.29 & 1.80 & 0.32 & 0.71 & 0.69 & 186 & 260 \\
\hline \multicolumn{11}{|l|}{ Statistics } \\
\hline Samples & & 17 & 17 & 17 & 17 & & & 17 & 13 & 13 \\
\hline Mean & & 363 & 0.29 & 0.99 & 1.29 & & & 0.71 & 109 & 328 \\
\hline Standard deviation & & 47 & 0.48 & 1.61 & 0.62 & & & 1.00 & 71 & 226 \\
\hline Median & & 374 & 0.04 & 0.18 & 1.13 & & & 0.27 & 103 & 260 \\
\hline \multicolumn{11}{|c|}{ Hole 654A Miocene (Tortonian) samples: } \\
\hline $41 \mathrm{R}-1,0-1$ & 357.81 & 355 & 0.01 & 0.06 & 1.89 & 0.17 & 0.03 & 0.25 & 24 & 756 \\
\hline $41 \mathrm{R}-1,70-73$ & 358.50 & 367 & 0.15 & 0.35 & 1.62 & 0.30 & 0.21 & 0.41 & 85 & 395 \\
\hline $41 \mathrm{R}-4,69-72$ & 363.30 & 491 & 0.00 & 0.20 & 1.68 & 0.00 & 0.11 & 0.29 & 68 & 582 \\
\hline $42 \mathrm{R}-1,69-72$ & 368.09 & 373 & 0.10 & 0.25 & 1.53 & 0.28 & 0.16 & 0.34 & 76 & 450 \\
\hline $42 R-4,61-64$ & 372.51 & 402 & 0.70 & 1.42 & 1.52 & 0.33 & 0.93 & 0.71 & 200 & 214 \\
\hline $42 R-6,21-24$ & 375.11 & 348 & 0.07 & 0.19 & 1.64 & 0.27 & 0.11 & 0.43 & 44 & 381 \\
\hline $42 \mathrm{R}-\mathrm{CC}, 6-8$ & 377.25 & 406 & 0.00 & 0.13 & 1.84 & 0.00 & 0.07 & 0.32 & 40 & 575 \\
\hline $43 R-2,107-110$ & 379.67 & 326 & 0.07 & 0.08 & 1.23 & 0.50 & 0.06 & 0.15 & 53 & 820 \\
\hline $43 \mathrm{R}-4,10-11$ & 381.70 & 436 & 0.01 & 0.05 & 1.45 & 0.17 & 0.03 & 0.15 & 33 & 966 \\
\hline $44 R-2,109-112$ & 389.39 & 327 & 0.00 & 0.05 & 1.03 & 0.00 & 0.04 & 0.23 & 21 & 447 \\
\hline $44 \mathrm{R}-5,119-120$ & 393.99 & 390 & 0.01 & 0.11 & 1.15 & 0.08 & 0.09 & 0.30 & 36 & 383 \\
\hline $44 R-6,96-99$ & 395.26 & 385 & 0.05 & 0.12 & 0.87 & 0.31 & 0.13 & 0.37 & 32 & 235 \\
\hline $45 \mathrm{R}-1,80-82$ & 397.20 & 411 & 0.04 & 0.24 & 0.65 & 0.14 & 0.36 & 0.42 & 57 & 154 \\
\hline $45 \mathrm{R}-6,0-1$ & 403.90 & 395 & 0.00 & 0.09 & 0.90 & 0.00 & 0.10 & 0.29 & 31 & 310 \\
\hline $45 \mathrm{R}-7,13-14$ & 405.53 & 341 & 0.00 & 0.06 & 0.77 & 0.00 & 0.07 & 0.13 & 46 & 592 \\
\hline \multicolumn{11}{|l|}{ Statistics } \\
\hline Samples & & 15 & 15 & 15 & 15 & & & 15 & 15 & 15 \\
\hline Mean & & 374 & 0.11 & 0.29 & 1.39 & & & 0.32 & 65 & 462 \\
\hline Standard deviation & & 37 & 0.22 & 0.46 & 0.39 & & & 0.19 & 59 & 246 \\
\hline Median & & 385 & 0.03 & 0.11 & 1.27 & & & 0.30 & 42 & 389 \\
\hline
\end{tabular}

Note: $\mathrm{T}_{\max }$ in ${ }^{\circ} \mathrm{C} ; \mathrm{S}_{1}$ and $\mathrm{S}_{2}$ in $\mathrm{mg}$ hydrocarbons $/ \mathrm{g}$ sediment; $\mathrm{S}_{3}$ in $\mathrm{mg} \mathrm{CO} 2 / \mathrm{g}$ sediment; PI in mg free hydrocarbons/g sediment; TOC in weight $\%$; $\mathrm{HI}$ in $\mathrm{mg}$ hydrocarbons $/ \mathrm{g}$ TOC; OI in $\mathrm{mg} \mathrm{CO} / \mathrm{g}$ TOC.

are more pronounced on the lower margin at Site 652; and (2) organic matter in the Pliocene is least abundant, least hydrogen-rich, and least mature at both sites.

\section{Origin and Maturity of Organic Matter from Lipid Analyses}

The amount of extractable organic matter in the sediments analyzed here is strongly influenced by the co-extraction of elemental sulfur during sample preparation. Therefore the calculation of the amount of extract per unit of organic carbon (Table 3) leads to numbers that exceed by far the theoretical possible quantities. Desulfurization of the hydrocarbon fractions of Samples 107-654-39R-1, 102-104 cm, and 107-654-39R-2, 6-9 cm, yielded a $1.5 \%-3 \%$ contribution of this compound class to the organic carbon pool.

Even in Sample 107-652-62R-1, 18-20 cm, which yielded the lowest amount of extract per $\mathrm{g}$ organic carbon $(234 \mu \mathrm{g} / \mathrm{g})$, the GC-MS analysis of the hydrocarbon fraction indicated a substantial amount of $\mathrm{S}_{8}$ in the lipid extract.
The gas-chromatographic analysis of the hydrocarbon fractions revealed considerable similarities among the set of samples studied. Most spectra are dominated by short-chain $n$-alkanes maximizing in the range of $n-\mathrm{C}_{15}$ to $n-\mathrm{C}_{19}$. There are a few exceptions like Sample 107-655-2H-6, 90-96 cm, however, that exhibit a strong odd preference in the long-chain $n$-alkanes. These compounds, in addition, are the dominant constituents of the total alkanes in immature samples (Figs. 8A through $8 \mathrm{~L}$ ).

Some characteristics of the hydrocarbon spectra employed to establish organic matter type are summarized in Table 4 . The carbon preference index is calculated according to Bray and Evans (1961) in the range of $n-C_{14}$ to $n-C_{20}$ and in the range of $n-C_{26}$ to $n-C_{32}$. This allows a general distinction between marine algal lipids and terrestrial plant lipids. The ratio of $n-\mathrm{C}_{27}+n$ $\mathrm{C}_{29}+n-\mathrm{C}_{31}$ divided by $n-\mathrm{C}_{15}+n-\mathrm{C}_{17}+n-\mathrm{C}_{19}$ ("Source Index," $I_{s}$ ) should help to assess the relative abundance of either short-chain lipidic material (values $\ll 1$ ) or long-chain lipidic material (values $>1$ ). In this context, short-chain lipidic material represents marine-derived organic matter, or indicates the 


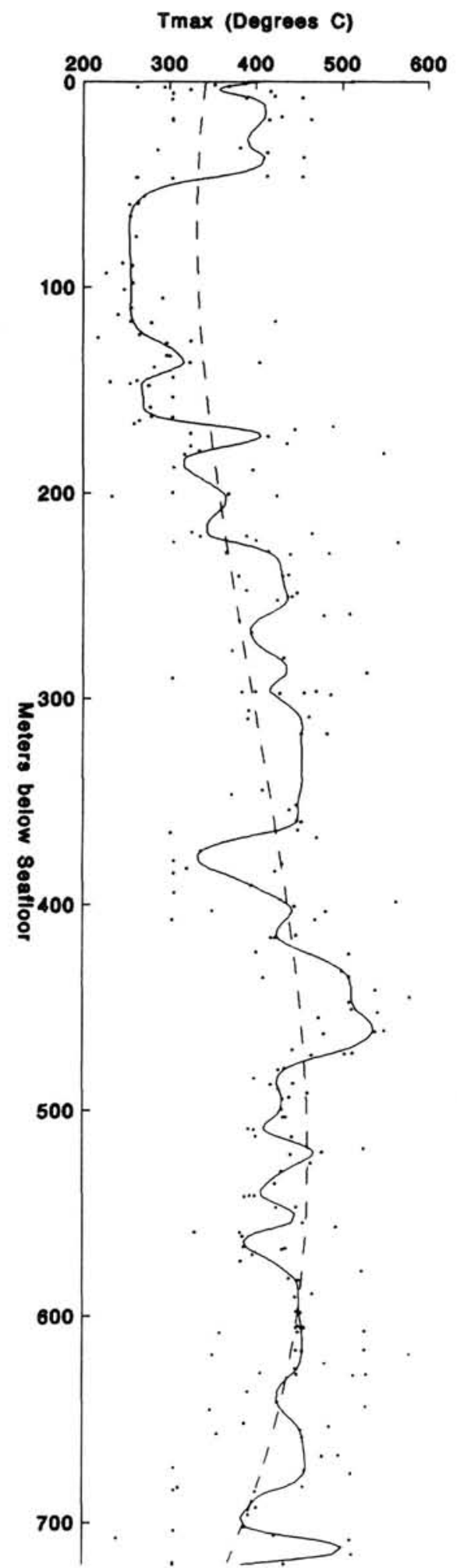

TOC (Weight \%)

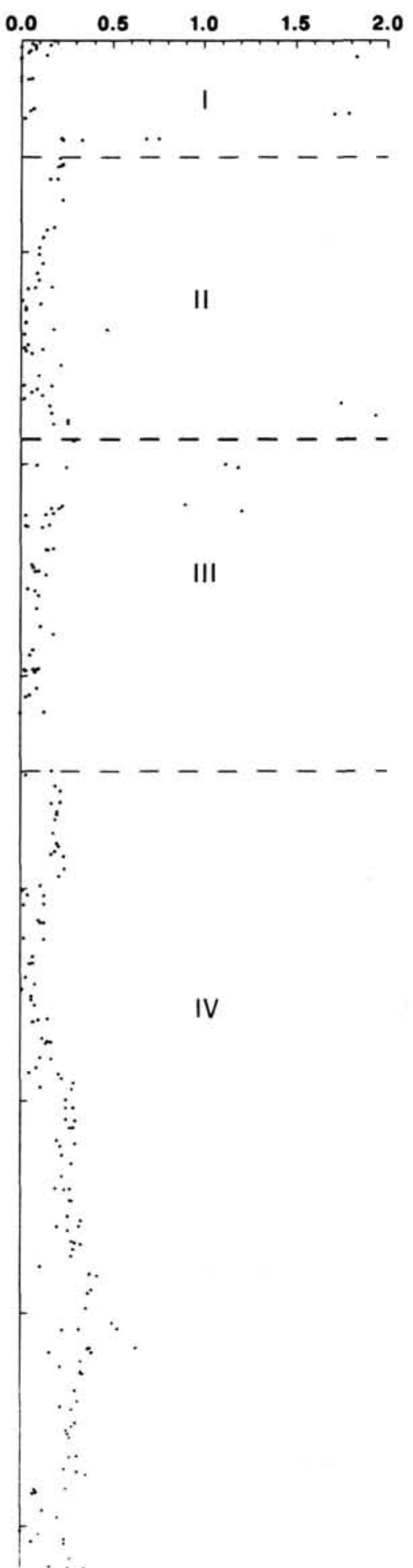

Hydrogen Index

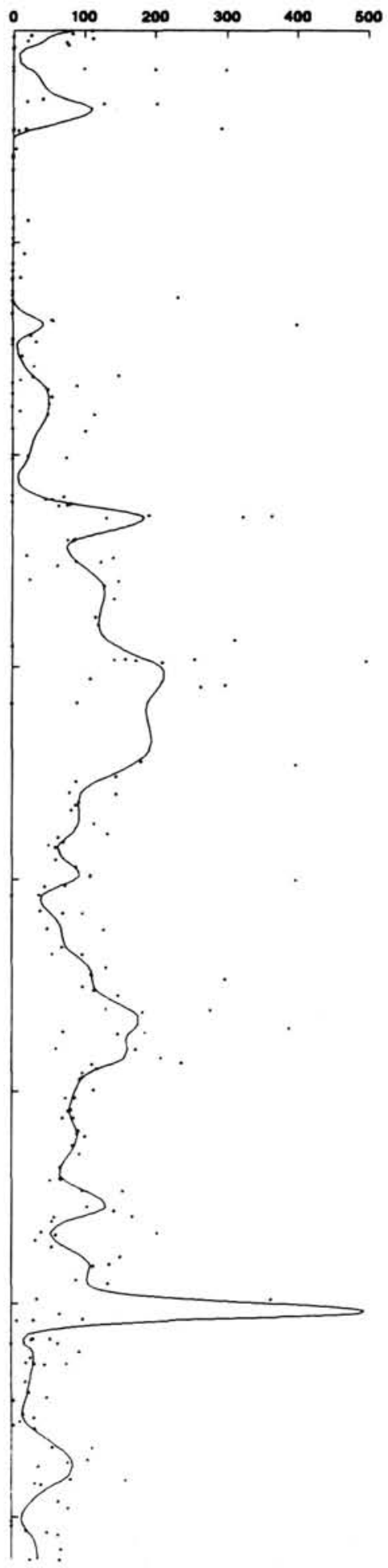

Figure 4. Organic matter abundance, hydrogen index, and maturity plots for Hole $652 \mathrm{~A}$. The central column shows lithologic units and TOC concentrations. Not shown are values above $2.0 \%$ TOC that occur in Units I and II (sapropels), and the oil shales in Unit V that reached up to $11 \%$ TOC. Solid line in the $\mathrm{T}_{\max }$ and hydrogen index columns is a filtered and smoothed curve; the broken line in the $\mathrm{T}_{\max }$ plot is a second-order polynomial regression line. 


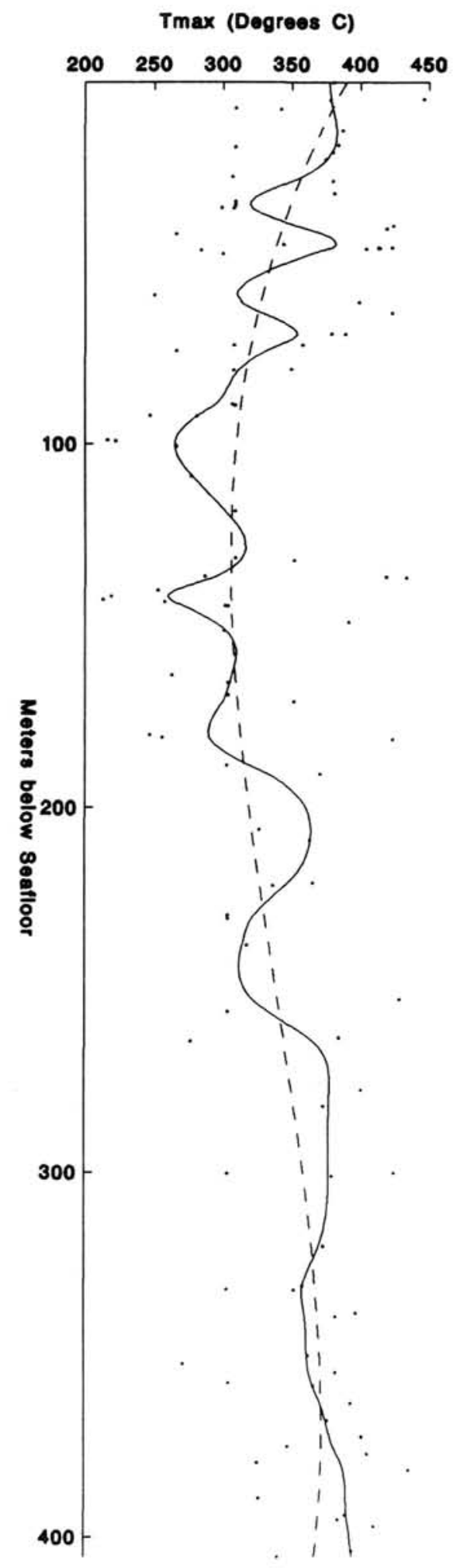

TOC (Woight \%)

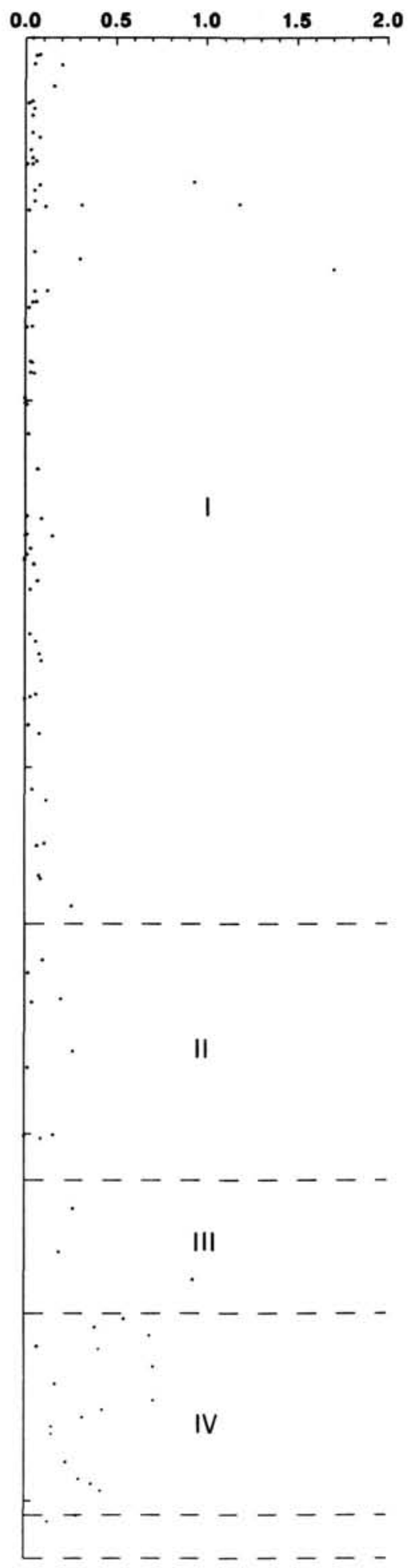

Hydrogen Index

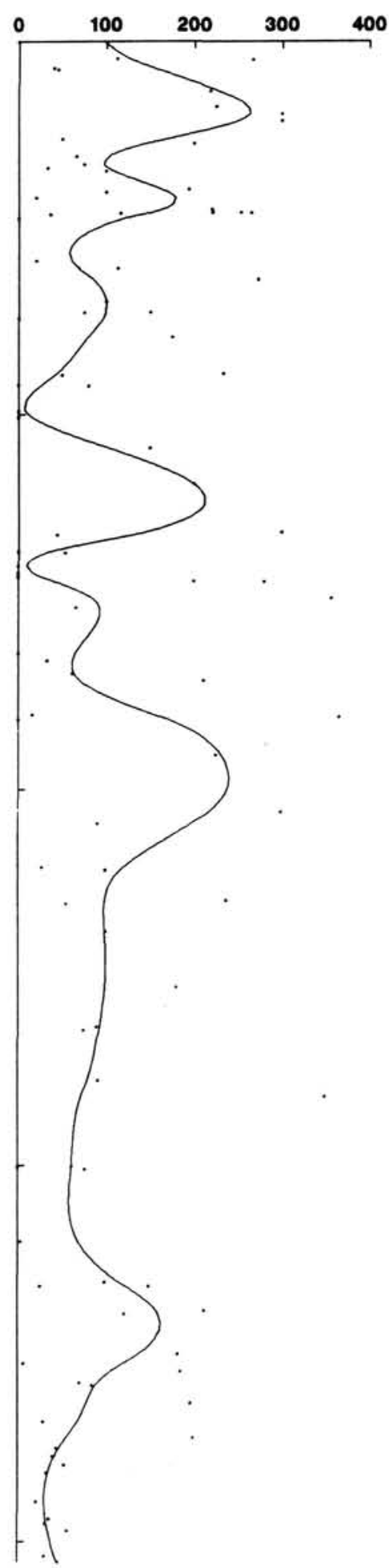

Figure 5. Organic matter abundance, hydrogen index, and maturity plots for Hole 654A. The central column shows lithologic units and TOC concentrations. Not shown are values above $2.0 \%$ TOC that occur in Units I (sapropels). Solid line in the $\mathrm{T}_{\max }$ and hydrogen index columns is a filtered and smoothed curve; the broken line in the $\mathrm{T}_{\max }$ plot is a second-order polynomial regression line. 

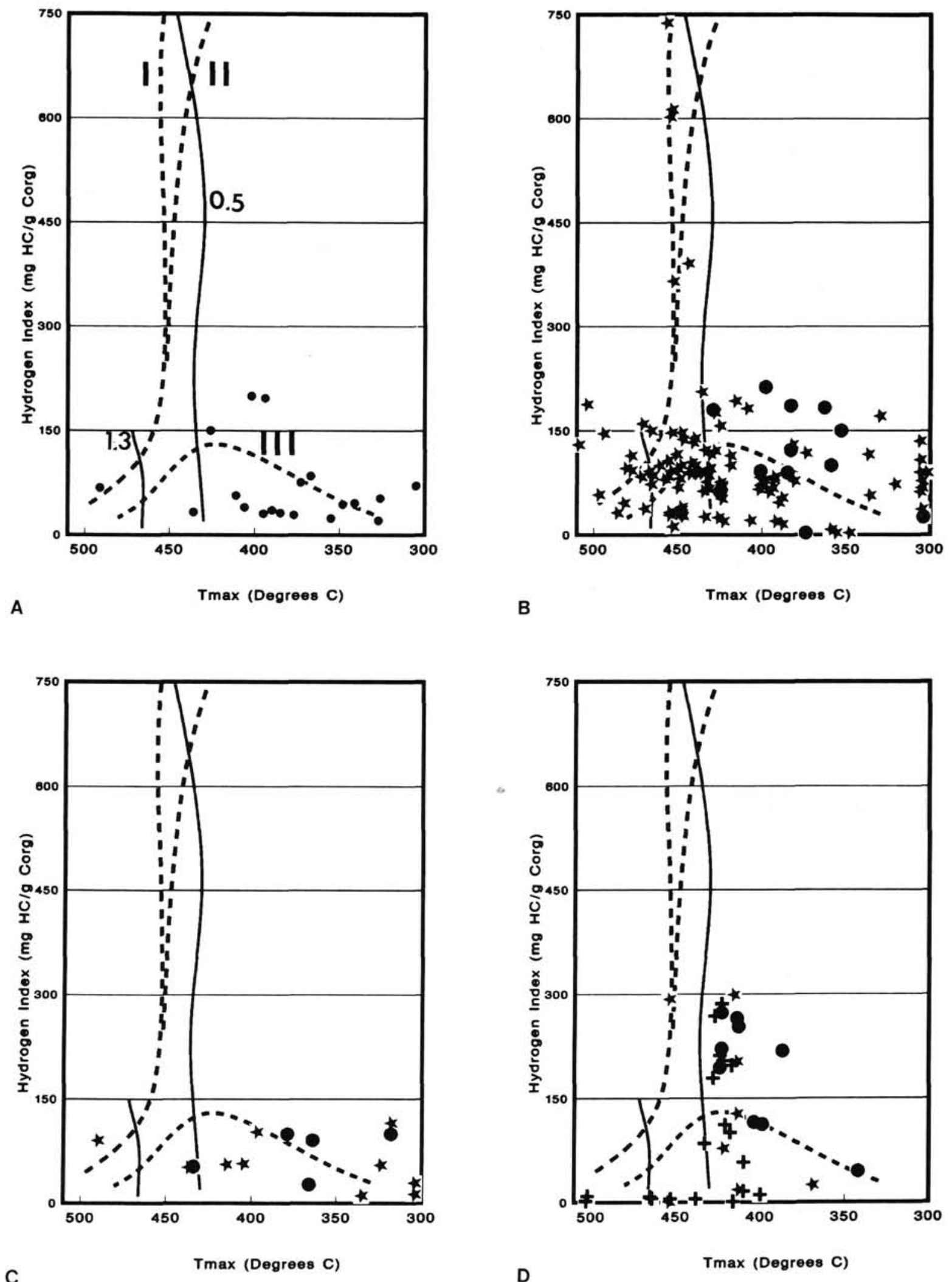

Figure 6. Results of pyrolysis for sediments of Tortonian (A), Messinian (B), Pliocene (C), and Pleistocene (D) age from Holes $652 \mathrm{~A}(*)$ and $654 \mathrm{~A}(\bullet)$. Samples are plotted in a diagram relating $\mathrm{T}_{\max }$ and $\mathrm{HI}$ to the origin and maturity of organic matter. Broken lines represent empirical maturation curves of three major classes of organic matter. Solid line at about $425^{\circ} \mathrm{C}$ represents the lower maturity boundary of the oil window at values equivalent to $0.5 \%$ Ro vitrinite reflectance. The solid line at approximately $470^{\circ} \mathrm{C}$ corresponds to approximately $1.3 \% \mathrm{Ro}$, the upper boundary of the oil window. Note the occurrence of Type I organic matter in (B) from Hole $652 \mathrm{~A}$, corresponding to the oil shale interval in Core -64R. Added are results for sapropel samples from Core 107$655 \mathrm{~A}-2 \mathrm{H}-4$ and surrounding nannofossil oozes $(+)$ for comparison of organic-rich and organic-poor sediments in the diagram for the Pleistocene. 


\section{Hole 652A}

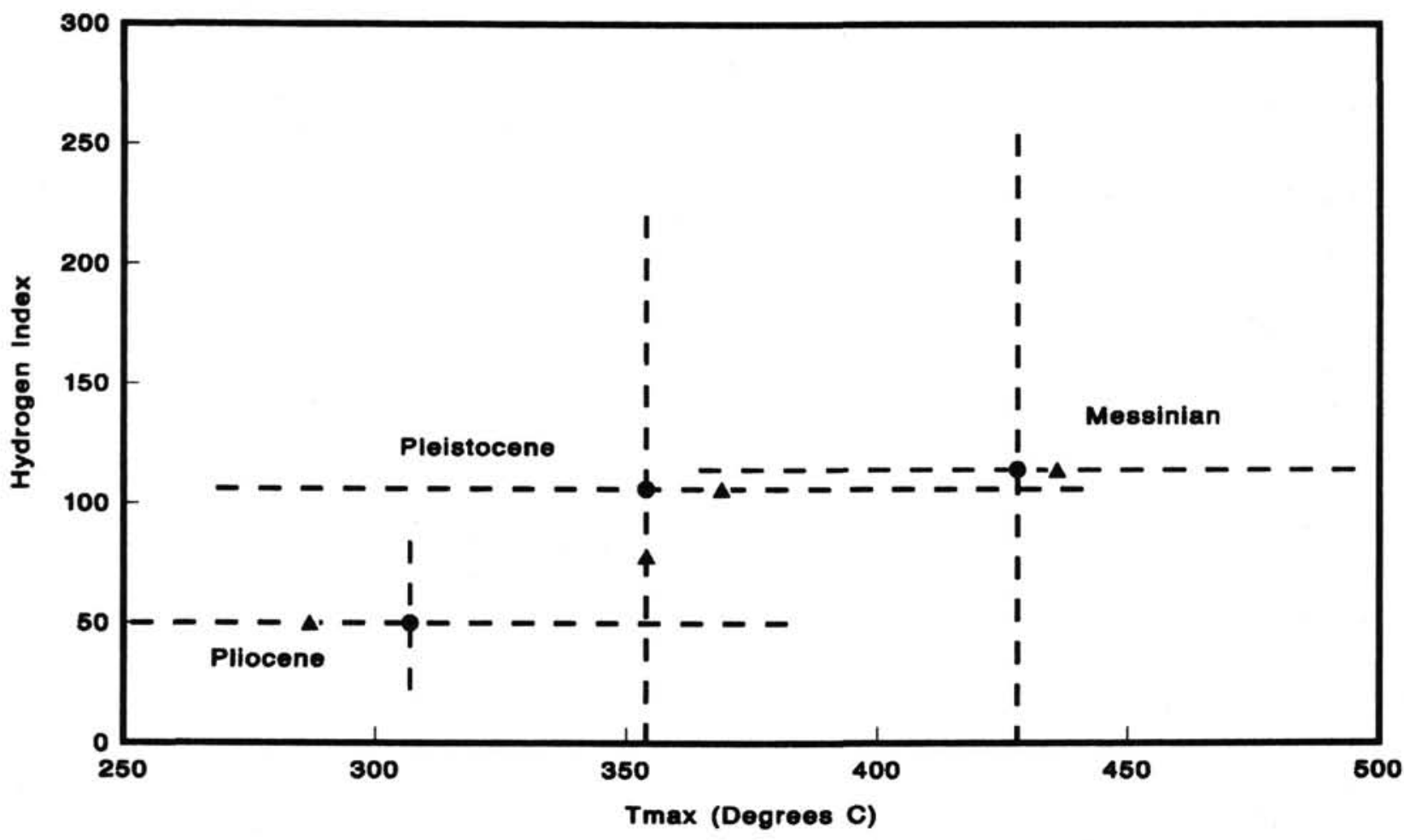

Hole 654A

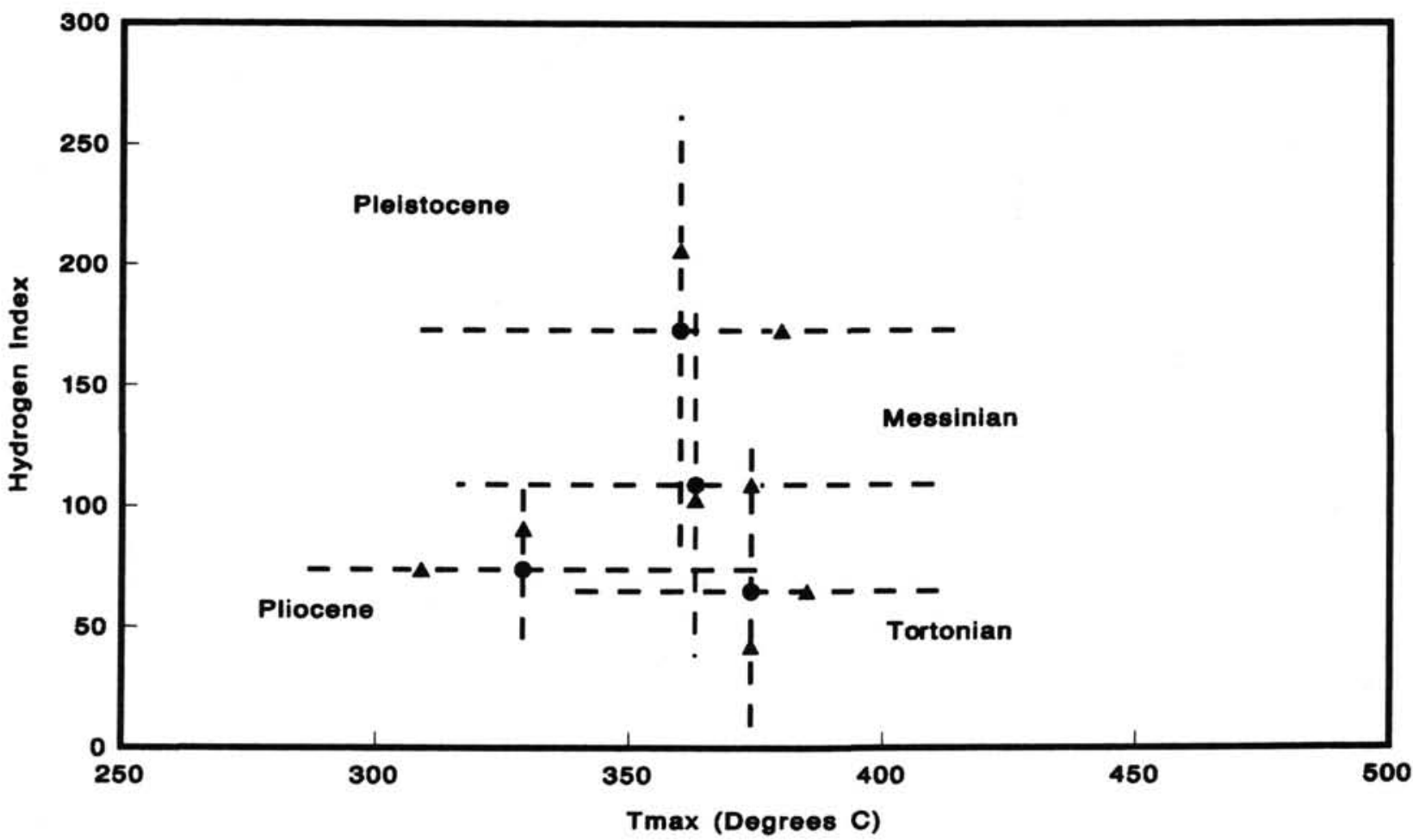

Figure 7. Summary diagrams of means (dots), standard deviations (dashed lines), and medians (triangles) of data plotted in Figures 6a-6d and listed in Tables 1 and 2. 
Table 3. Extract quantities of Messinian sediments, Leg 107.

\begin{tabular}{|c|c|c|c|c|c|c|}
\hline \multirow{2}{*}{$\begin{array}{l}\text { Core, section, } \\
\text { interval }(\mathrm{cm})\end{array}$} & \multirow{2}{*}{$\begin{array}{l}\text { TOC } \\
(\%)\end{array}$} & \multicolumn{2}{|c|}{ Extract $(\mu \mathrm{g}) /$} & \multicolumn{2}{|c|}{ HC-Sulfur ( $\%)$} & \multirow{2}{*}{$\underset{(\%)}{S}$} \\
\hline & & g sed. & gTOC & $\mathrm{g}$ sed. & gTOC & \\
\hline \multicolumn{7}{|l|}{$107-652 \mathrm{~A}-$} \\
\hline${ }^{a} 58 R-5,67-69$ & 0.28 & 18.7 & 6694 & - & - & - \\
\hline${ }^{a} 59 R-2,140-150$ & 1.00 & 10.4 & 1037 & - & - & - \\
\hline${ }^{a} 62 R-1,18-20$ & 0.37 & 0.9 & 234 & - & - & - \\
\hline a $63 R-3,40-42$ & 1.98 & 14.0 & 705 & - & - & - \\
\hline${ }^{\mathrm{a}} 63 \mathrm{R}, \mathrm{CC}, 17-19$ & 3.07 & 20.4 & 665 & - & - & - \\
\hline $64 \mathrm{R}-1,75-77$ & 2.51 & 14.3 & 570 & - & - & - \\
\hline $65 \mathrm{R}-6,105-108$ & 0.33 & 6.8 & 2058 & - & - & - \\
\hline \multicolumn{7}{|l|}{$107-654 \mathrm{~A}-$} \\
\hline a $38 \mathrm{R}-1,126-132$ & 1.45 & 17.6 & 1215 & - & - & - \\
\hline${ }^{\mathrm{a}} 38 \mathrm{R}-2,33-39$ & 3.21 & 13.3 & 413 & - & - & 6.86 \\
\hline $39 \mathrm{R}-1,7-10$ & 2.18 & 21.4 & 981 & - & - & 2.58 \\
\hline $39 \mathrm{R}-1,102-104$ & 1.01 & 11.6 & 1146 & 0.03 & 3.01 & - \\
\hline $39 \mathrm{R}-2,6-9$ & 2.36 & 47.7 & 2020 & 0.04 & 1.55 & - \\
\hline \multicolumn{7}{|l|}{$107-655 \mathrm{~A}$ - } \\
\hline $\begin{array}{c}\text { 2H-6, } 90-96 \\
\text { (sapropel } \\
\text { sample) }\end{array}$ & 0.82 & 14.5 & 1770 & - & - & - \\
\hline
\end{tabular}

a Samples analyzed by GC-MS.

onset of petroleum generation. Long-carbon-chain lipids derive from terrigenous material in most cases. Ratios of pristane to $n$ $\mathrm{C}_{17}$, phytane to $n-\mathrm{C}_{18}$, the pristane to phytane ratio, and other carbon preference indexes (CPI) that cover the alkanes from carbon-chain lengths from $\mathrm{C}_{14}$ to $\mathrm{C}_{32}$ are also given in Table 4 .

The ratios of pristane to phytane in four of the analyzed sediments yielded values below unity. The closely spaced samples from 107-652A-63R-CC, $17-19 \mathrm{~cm}$, to $107-652 \mathrm{~A}-64 \mathrm{R}-1,75-77$ $\mathrm{cm}$, bracket oil-shale sediments deposited during a local anoxic event in a relatively short time span. The observed pristane/phytane ratio may have originated from the degradation of the phytanyl side chain of chlorophyll as suggested by Didyk et al. (1978).

The ratios of pristane to $n-\mathrm{C}_{17}$ and phytane to $n-\mathrm{C}_{18}$ vary from 0.07 to around 1. The lower value of 0.07 is found in the deepest Sample 107-652A-65R-6 (623 mbsf), whereas those around 1 are found at depths of around $300 \mathrm{~m}$ in Hole 654A. The samples with the lowest values are dominated by shortchain alkanes and hence have the lowest value of $I_{s}$ (Table 4).

In samples of comparable kerogen type, Connan and Cassou (1980) noted a steady decrease in the ratios of $\mathrm{pr} / n-\mathrm{C}_{17} \mathrm{vs}$. ph/ $n-\mathrm{C}_{18}$ with increasing maturity. A plot of the $\mathrm{pr} / n-\mathrm{C}_{17}$ vs. $\mathrm{ph} / n-$ $\mathrm{C}_{18}$ ratios (Fig. 9) indicates a very close relationship of all the samples analyzed (Hole 652A, 654A, and 655A). They scatter around a line that advertises them as members of the algal kerogen Type II (Connan and Cassou, 1980). The ratio of pristane to phytane never exceeds a value of 1.75 and is an additional proof for the algal origin of organic matter in the samples (Table 4). Pristane to phytane ratios of lower than 1.5 are generally encountered in carbonates, evaporites, and marine sediments with kerogen Type I (Powell and McKirdy, 1973; Connan et al., 1986). These indicators provide additional evidence for the origin of the oil-shale samples from Hole 652A and the sapropel sample (107-655A-2H-6, 90-96 cm).

The five samples that yield $\mathrm{pr} / n-\mathrm{C}_{17}$ ratios below 0.3 and $\mathrm{ph} / n-\mathrm{C}_{18}$ below 0.6 (Fig. 9) can be regarded as mature oil source rocks. The same set of samples plot closely around the origin of the axes in a pr/n- $C_{17}$ vs. $I_{s}$ graph (Fig. 10). This graph illustrates the effect of maturation on the samples, when short-chain $n$-alkanes are continuously produced.
A number of biomarker compounds was investigated and characterized by GC-MS of the lipid extracts. The alkylcyclohexanes were found in significant abundances in all samples studied (Figs. 11A-11G). The cyclohexanes are present up to carbon numbers of 30 . Their maximum usually falls in the region of $\mathrm{C}_{17}$ or $\mathrm{C}_{18}$, but a second maximum around $\mathrm{C}_{20}$ or $\mathrm{C}_{23}$ is often observed. No significant depth or maturity trend is discernable in Hole 652. Sample 107-652A-59R-2, 140-150 cm, exposes an unusual even predominance of the cyclohexanes with a dominance of $\mathrm{C}_{18}$ and $\mathrm{C}_{20}$ compounds (Fig. 11D). A comparable even predominance was found in the $n$-alkane distribution spectrum of this sample as well, although it is not as pronounced (Table 4; Fig. 8F).

The two samples of Hole 654A are clearly odd-predominated in their cyclohexane carbon-number distribution (Figs. 11A and 11B). Sample 107-654A-38R-1, 126-132 cm, displays the strongest odd preference, maximizing at the $C_{21}$ cyclohexane. Such a strong odd-over-even predominance is a result of thermal immaturity in samples from Hole 654A (Figs. 11A and 11B), an assumption that is corroborated by the lack of comparable oddeven preference in mature samples (e.g., Sample 107-652A-63R-3, 40-42 cm; Fig. 11F).

The biogenic signal in the distribution of alkylcyclohexanes is thus obscured by maturation processes. This observation is in keeping with the maturity-related changes observed in the $n$-alkane distribution (Figs. 8A through $8 \mathrm{~L}$ ). Another indication of maturity is the absence of unsaturated hydrocarbons. In the set of samples that has been investigated here by GC-MS, those from Hole 654A, notably Samples 107-654A-38R-1, 126-132 cm, and 107-654A-38R-2, 33-39 cm, showed significant contributions of diasterenes and spirosterenes. The occurrence of these unsaturated compounds may be explained by the abundance of clay minerals in the Messinian sequences that could provide acidic sites for the formation of carbocations. Hence, the transformation of sterenes to diasterenes (Rubinstein et al., 1978) or spirosterenes could follow. Spirosterenes may result as the products of backbone rearrangement of $\Delta_{4^{-}}$and $\Delta_{5}$-sterenes (Peakman et al., 1984). The $20 \mathrm{R}$ isomers are the predominant constituents in immature sediments while the $20 \mathrm{~S}$ isomer becomes increasingly abundant with increasing thermal maturity. The formation and isomerization reactions are thought to require acidic catalysis within the sediment (Peakman et al., 1984; Brassell et al., 1984).

A compound eluting directly before the pristane peak could be identified as 2,6,10-trimethyl-7-(3-methylbutyl)-dodecane (Yon et al., 1982). It has been found previously in various hypersaline environments (Robson and Rowland, 1986) and can be used among other evidence as an indicator for paleosalinity (ten $\mathrm{Ha}$ ven et al., 1987).

Sample 107-654A-38R-2, 33-39 cm, yielded 3-(4,8,12-trimethyltridecyl)-thiophene, a compound indicative of thermal immaturity of this sample. The compound is formed by the early diagenetic introduction of sulfur into phyta-1,3(17)-diene or phytol (Brassell et al., 1986). Compounds of this type have been reported from several DSDP sites (e.g., Leg 75, Angola Basin; Rullkötter et al., 1984) and may indicate an environment of either high sulfur abundance (evaporitic environment) or low iron abundance. Iron may alternatively to the organic matter act as a sink for sulfur during pyrite formation.

\section{Pentacyclic Biological Marker Compounds}

Hopane distributions are similar in three of the samples from Hole 652A (107-652A-58R-5, 67-69 cm (Fig. 12); 107-652A59R-2, 140-150 cm (Fig. 13); and 107-652A-62R-1, 18-20 cm (Fig. 14)). Their common and conspicuous feature is the occurrence of $18 \alpha(\mathrm{H})$ trisnorhopane, $17 \alpha(\mathrm{H})$ trisnorhopane, and 
$655-2 \mathrm{H}-6,90-96$

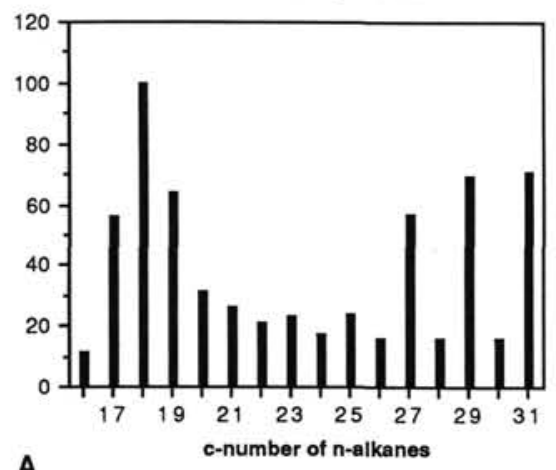

A

654-39R-2, 6-9

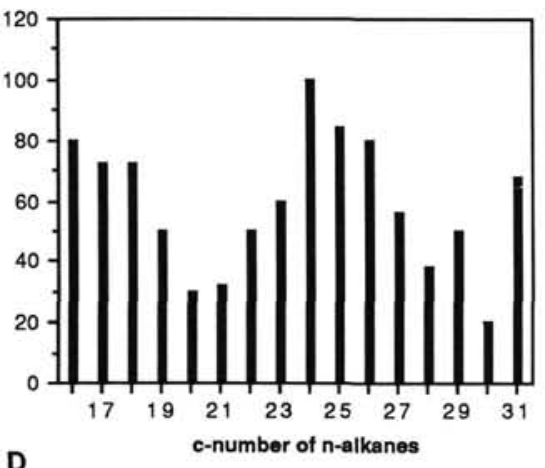

652-59R-2, 140-150

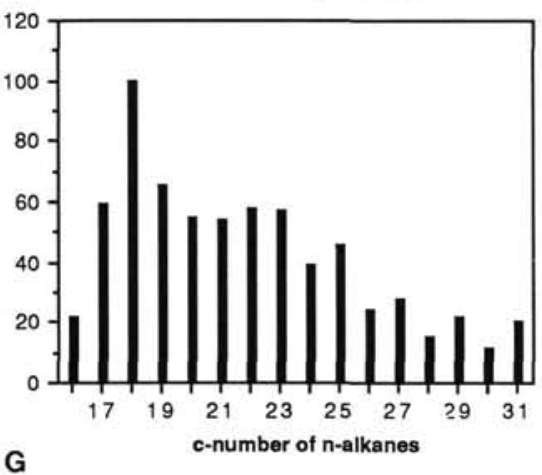

652-63R-cc, 17-19

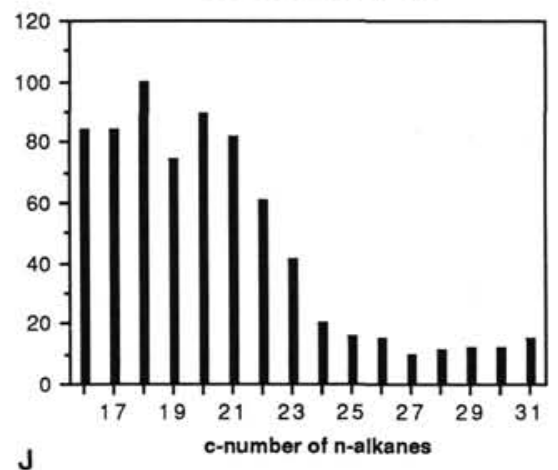

654-38R-1, 126-132

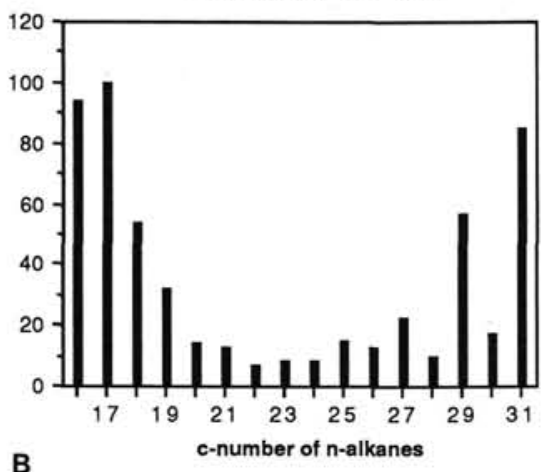

654-39R-1, 102-104

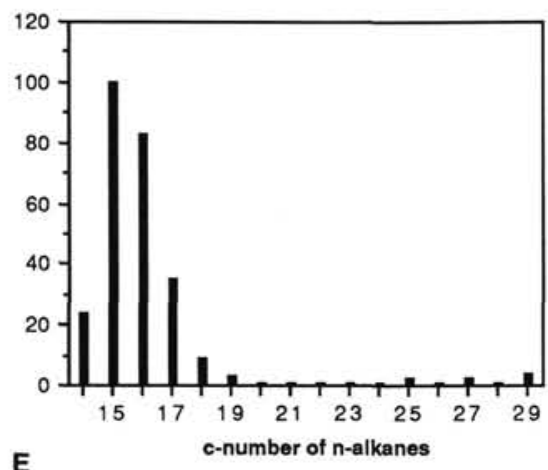

652-62R-1, 18-20

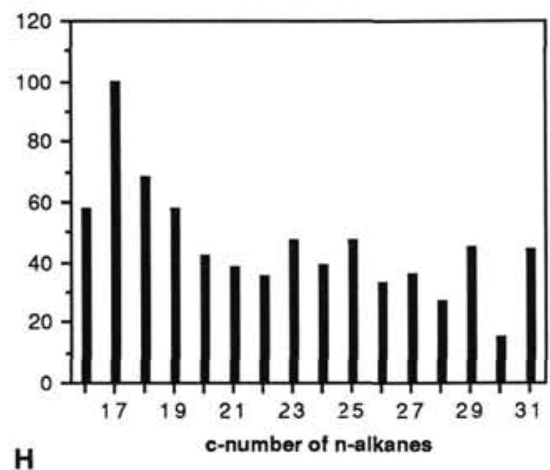

652-64R-1, 75-77

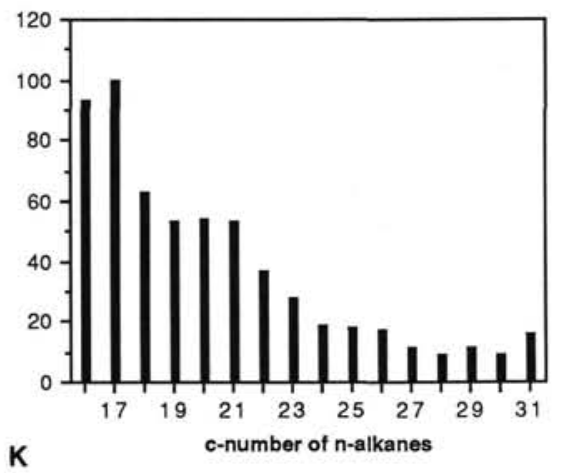

654-39R-1, 7-10

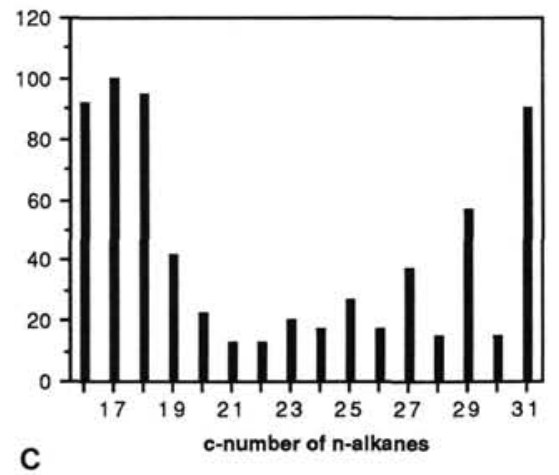

652-58R-5, 67-69

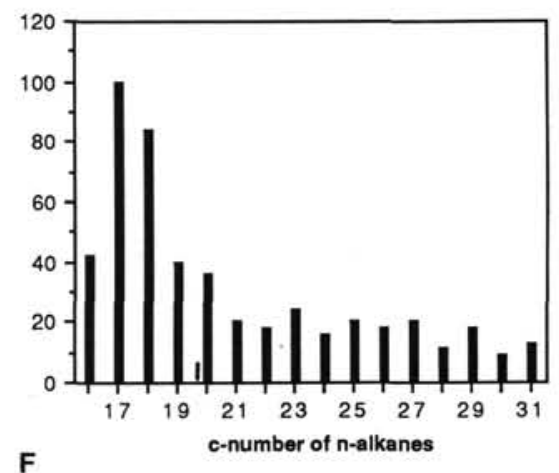

$652-63 R-3,40-42$

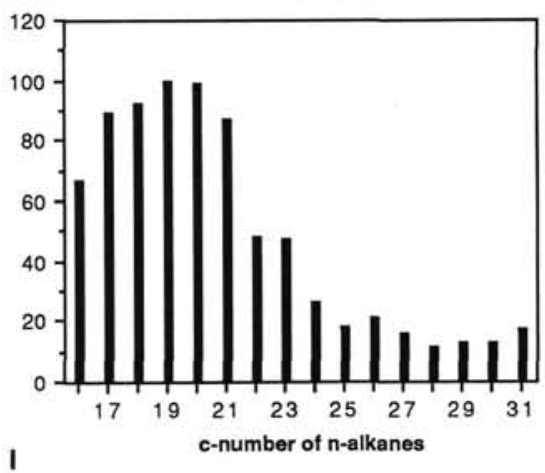

652-65R-6, 105-108

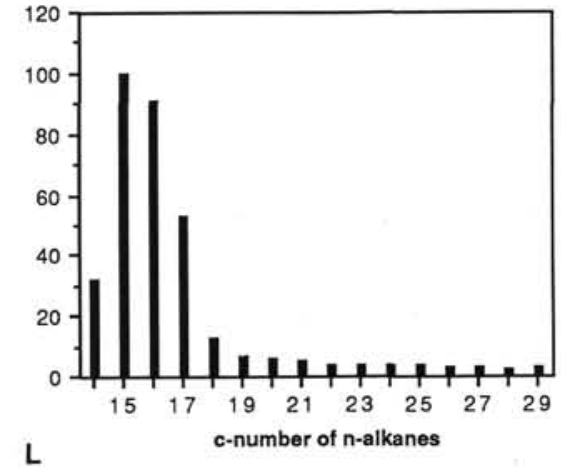

Figure 8. Carbon number distribution of $n$-alkanes in the sediments of the Tyrrhenian Sea ODP Leg 107. Distributions are shown between $\mathrm{C}_{16}$ and $\mathrm{C}_{31}$ with the exception of Samples 107-654-39R-1, 102-104 cm (Fig. 8E), and Sample 107-652A-65R-6, 105-108 cm (Fig. 8L), where the majority of $n$ alkanes is in the low-molecular-weight range. In these cases the interval of $\mathrm{C}_{14}-\mathrm{C}_{29}$ is monitored. 
Table 4. Characteristic ratios of compounds in lipid extracts for Messinian samples of Holes 652A and 654A. See text for discussion.

\begin{tabular}{lcccccc}
\hline $\begin{array}{c}\text { Core, section, } \\
\text { interval (cm) }\end{array}$ & Prist/C 17 & Phyt/ $C_{18}$ & Prist/Phyt & CPI $_{14-20}$ & CPI $_{26-32}$ & $I_{s}$ \\
\hline 107-652A- & & & & & & \\
& & & & & & \\
58R-5, 67-69 & 0.37 & 0.29 & 1.55 & 1.09 & 1.76 & 0.33 \\
59R-2, 140-150 & 0.59 & 0.22 & 1.75 & 0.94 & 1.81 & 0.51 \\
62R-1, 18-20 & 0.50 & 0.44 & 1.65 & 1.23 & 2.08 & 0.62 \\
63R-3, 40-42 & 0.22 & 0.31 & 0.70 & 1.06 & 1.20 & 0.21 \\
63R-CC, 17-19 & 0.20 & 0.38 & 0.44 & 0.94 & 1.07 & 0.16 \\
64R-1, 75-77 & 0.13 & 0.47 & 0.45 & 1.23 & 1.25 & 0.16 \\
65R-6, 105-108 & 0.07 & 0.22 & 1.23 & 1.34 & 1.72 & 0.05 \\
107-654A- & & & & & & \\
38R-1, 126-132 & 0.92 & 1.07 & 1.60 & 1.28 & 4.40 & 0.70 \\
38R-2, 33-39 & - & - & 1.51 & - & - & - \\
39R-1, 7-10 & 0.78 & 0.52 & 1.57 & 0.97 & 4.46 & 0.93 \\
39R-1, 102-104 & 0.19 & 0.48 & 1.50 & 1.34 & 3.79 & 0.08 \\
39R-2, 6-9 & 0.69 & 0.67 & 1.20 & 1.00 & 1.92 & 1.00 \\
107-655A- & & & & & & \\
2H-6, 90-96 & 0.55 & 0.42 & 0.76 & 1.02 & 4.16 & 1.49 \\
$\quad$ (sapropel & & & & & & \\
sample) & & & & & & \\
\hline
\end{tabular}

Note: $\mathrm{I}_{\mathrm{s}}=$ source index $=\mathrm{n}-\mathrm{C}_{27}+n-\mathrm{C}_{29}+n-\mathrm{C}_{31} / n-\mathrm{C}_{15}+n-\mathrm{C}_{17}+n-\mathrm{C}_{19}$. For details on CPI (Carbon Preference Index) see text. Prist $=$ Pristane, Phyt $=$ Phytane.

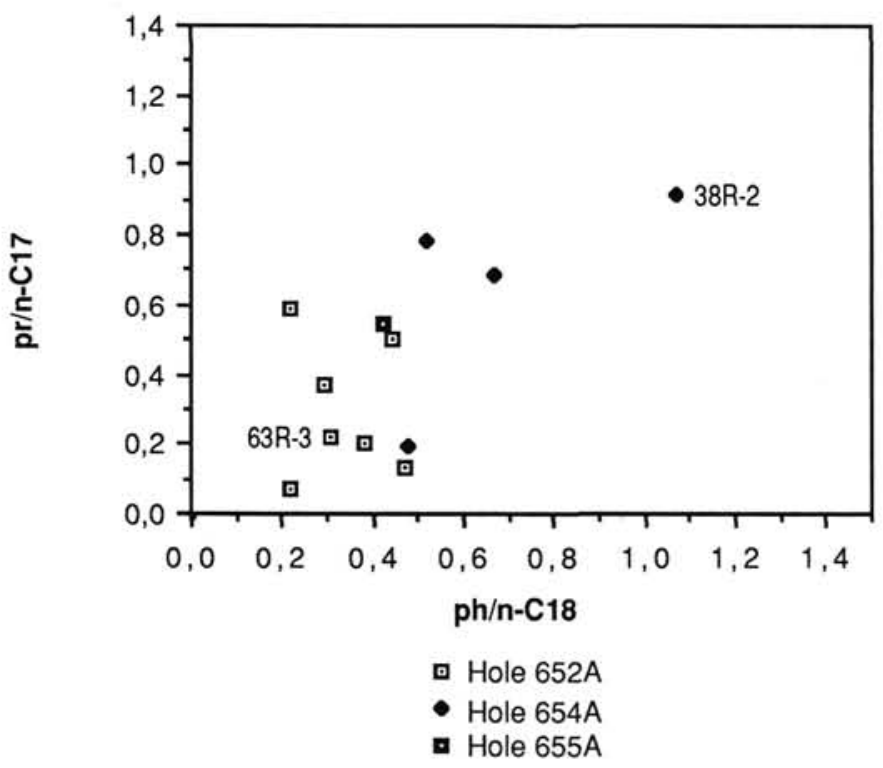

Figure 9. Diagram of $\mathrm{pr} / n-\mathrm{C}_{17}$ vs. $\mathrm{ph} / n-\mathrm{C}_{18}$, according to Connan and Cassou (1980), indicates the varying degrees of maturity in samples from Holes 652A and 654A.

$17 \alpha(\mathrm{H}), 21 \beta(\mathrm{H})$ bisnorhopane in the order of elution, indicating mature samples of hypersaline origin. In addition to the predominant series of $17 \alpha(\mathrm{H}), 21 \beta(\mathrm{H})$ hopanes, moretanes are observed in these samples. The $17 \alpha(\mathrm{H}), 21 \beta(\mathrm{H})$ hopane is the major component in all three spectra. The isomerization of $22 \mathrm{~S} / 22 \mathrm{~S}$ $+22 \mathrm{R}$ is around 0.64 for the $\mathrm{C}_{31}$ compound.

Samples 107-652A-63R-3, 40-42 cm (Fig. 15), and 107-652A63R-CC, $17-19 \mathrm{~cm}$ (Fig. 16), of the same hole are slightly different in their hopane distribution in that they expose only $17 \alpha(\mathrm{H})$ trisnorhopane at $\left(\mathrm{T}_{\mathrm{m}}\right)$. No $\mathrm{T}_{\mathrm{s}}$ was detectable, nor was there a trace of $17 \alpha(\mathrm{H}), 21 \beta(\mathrm{H})$ bisnorhopane, which indicates a different depositional environment for the oil shale samples. The

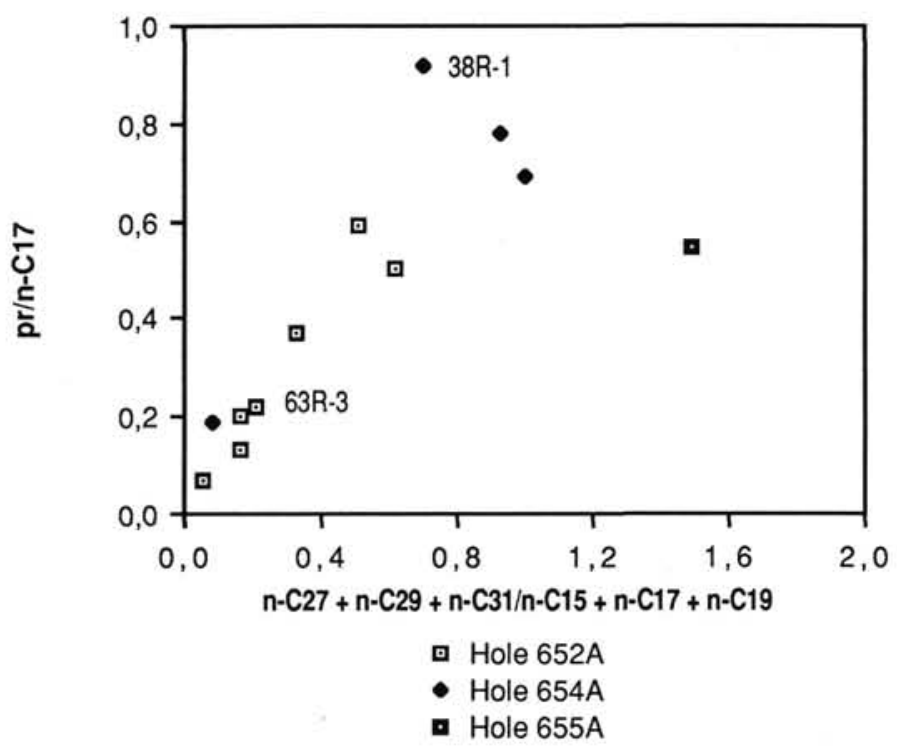

Figure 10. Diagram of $\mathrm{pr} / n-\mathrm{C}_{17}$ vs. source index $\mathrm{I}_{\mathrm{s}}$ (for explanation refer to text), which illustrates the maturity of those samples that fall close to the origin of the axes. Production of short-chain $n$-alkanes $\left(C_{17}\right)$ in the oil window underlines the observed trend in the diagram by lowering both the $\mathrm{pr} / n-\mathrm{C}_{17}$ and the source index.

$22 \mathrm{~S} / 22 \mathrm{~S}+22 \mathrm{R}$ isomerization of homohopane is 0.55 and 0.30 , respectively, indicating a lower maturity of these two samples, even though they are the deepest samples of Hole 652A. Few tricyclic compounds were observed in the electron impact-mass spectra $(\mathrm{M} / \mathrm{Z}) 191$ scans. The spectra are too weak to be interpreted.

Samples from Hole 654A (107-654A-38R-1, 126-132 cm (Fig. 17), and 107-654A-38R-2, 33-39 cm (Fig. 18)) appear to be rather immature based on the data from cycloalkanes and the pristane $/ n-C_{17}$ vs. $I_{s}$ plot (Fig. 10). These samples accordingly 
654-38R-1, 126-132

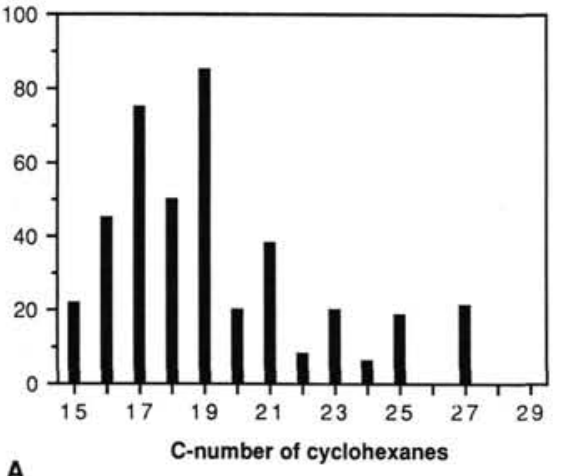

652-59R-2, $140-150$

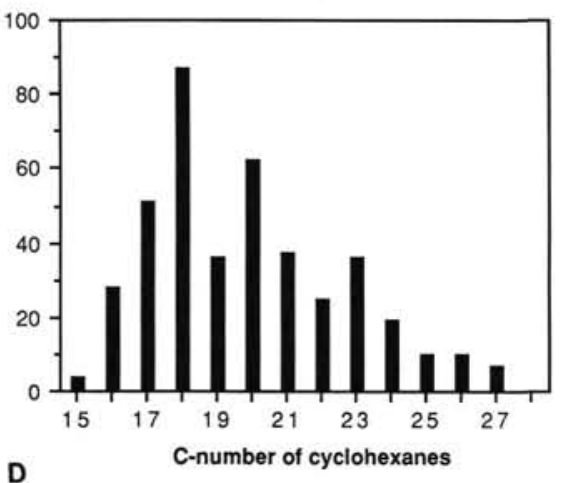

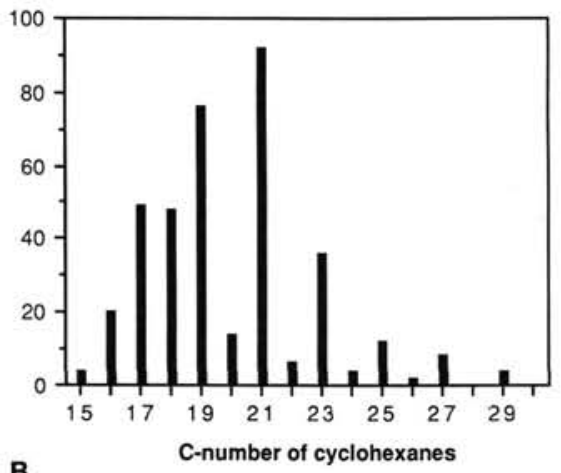

652-62R-1, 18-20

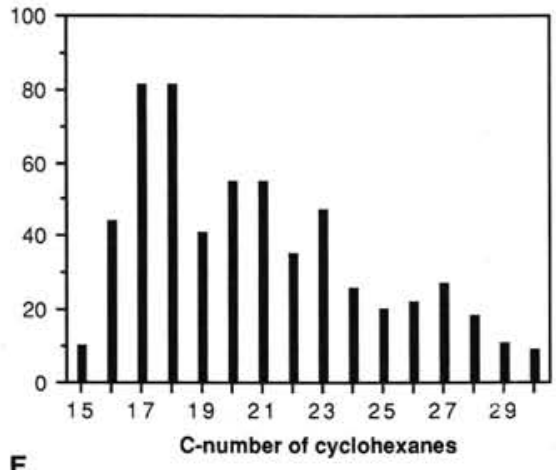

652-63R-cc, $17-19$

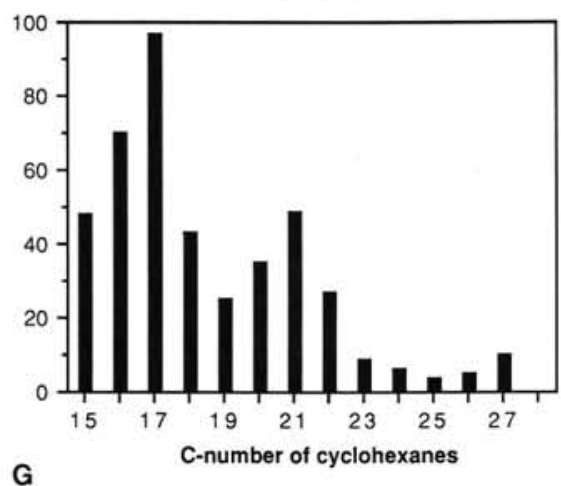

652-58R-5, 67-69

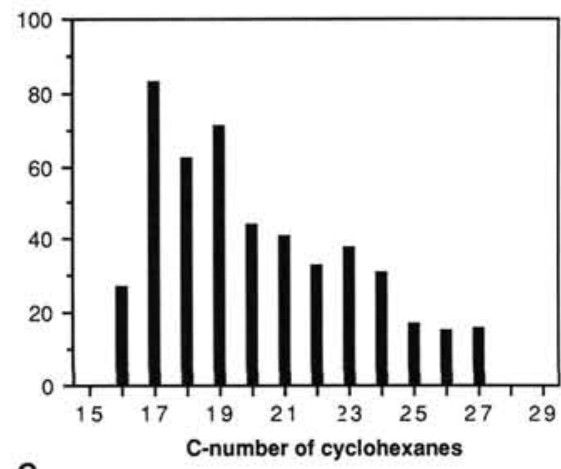

$652-63 R-3,40-42$

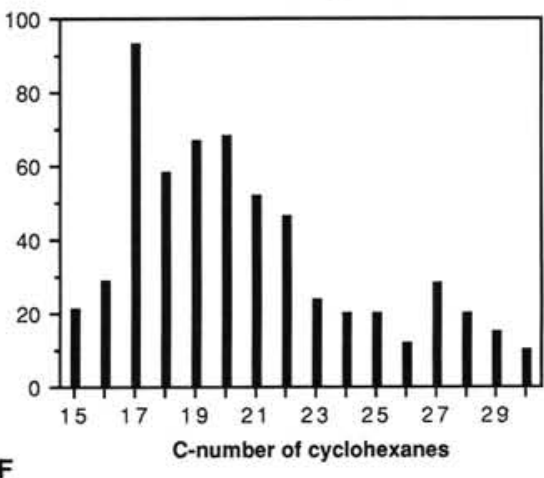

$\mathbf{F}$

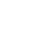



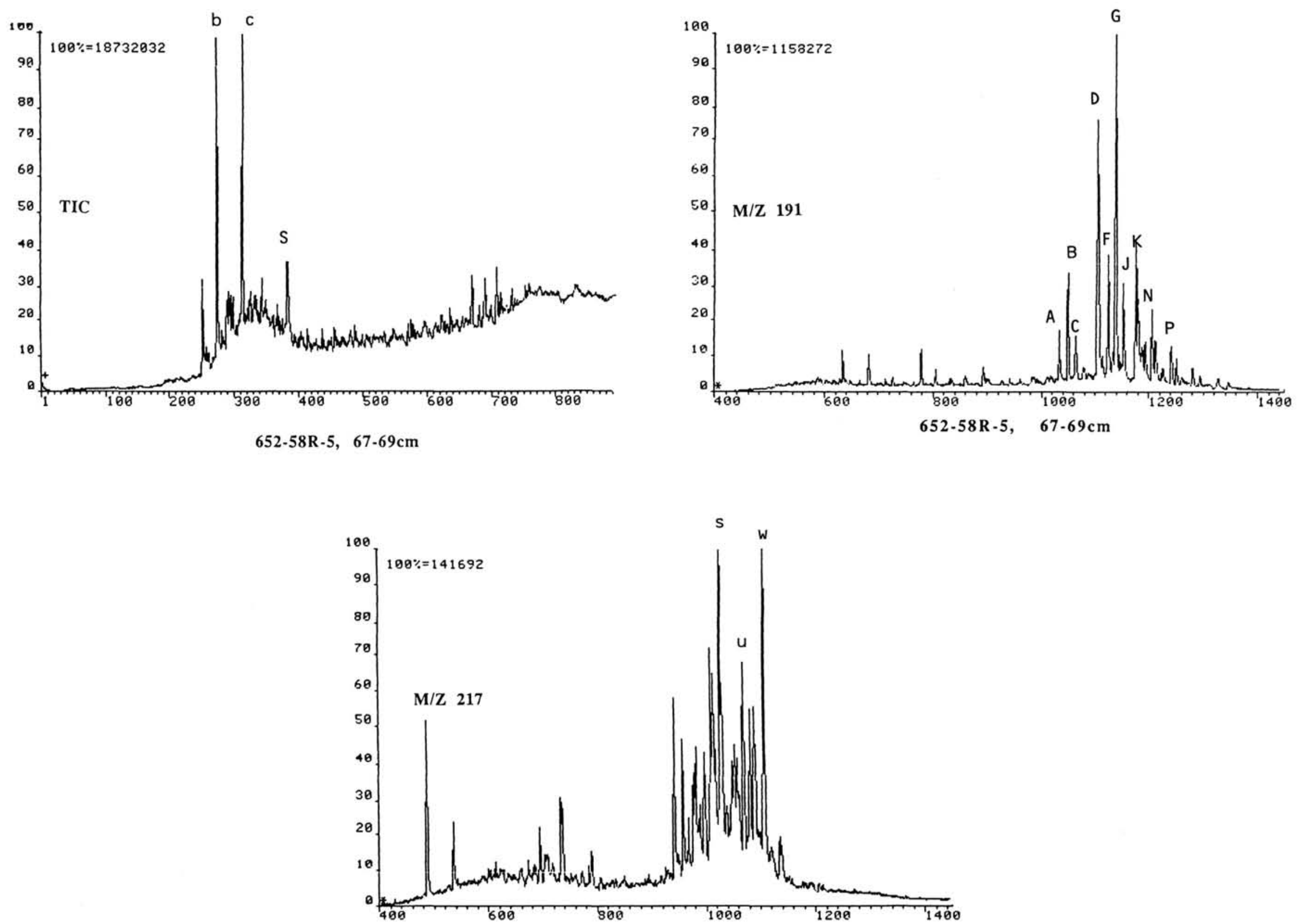

$652-58 \mathrm{R}-5,67-69 \mathrm{~cm}$

Figure 12. TIC (total ion current), M/Z 191 (diagnostic ion for the hopane family), and M/Z 217 (diagnostic ion for the sterane family) of the branched and cyclic fraction of Sample 107-652A$58 \mathrm{R}-5,67-69 \mathrm{~cm}$. For identification of assigned peaks refer to Table 6; for GC-MS conditions refer to the analytical section. 

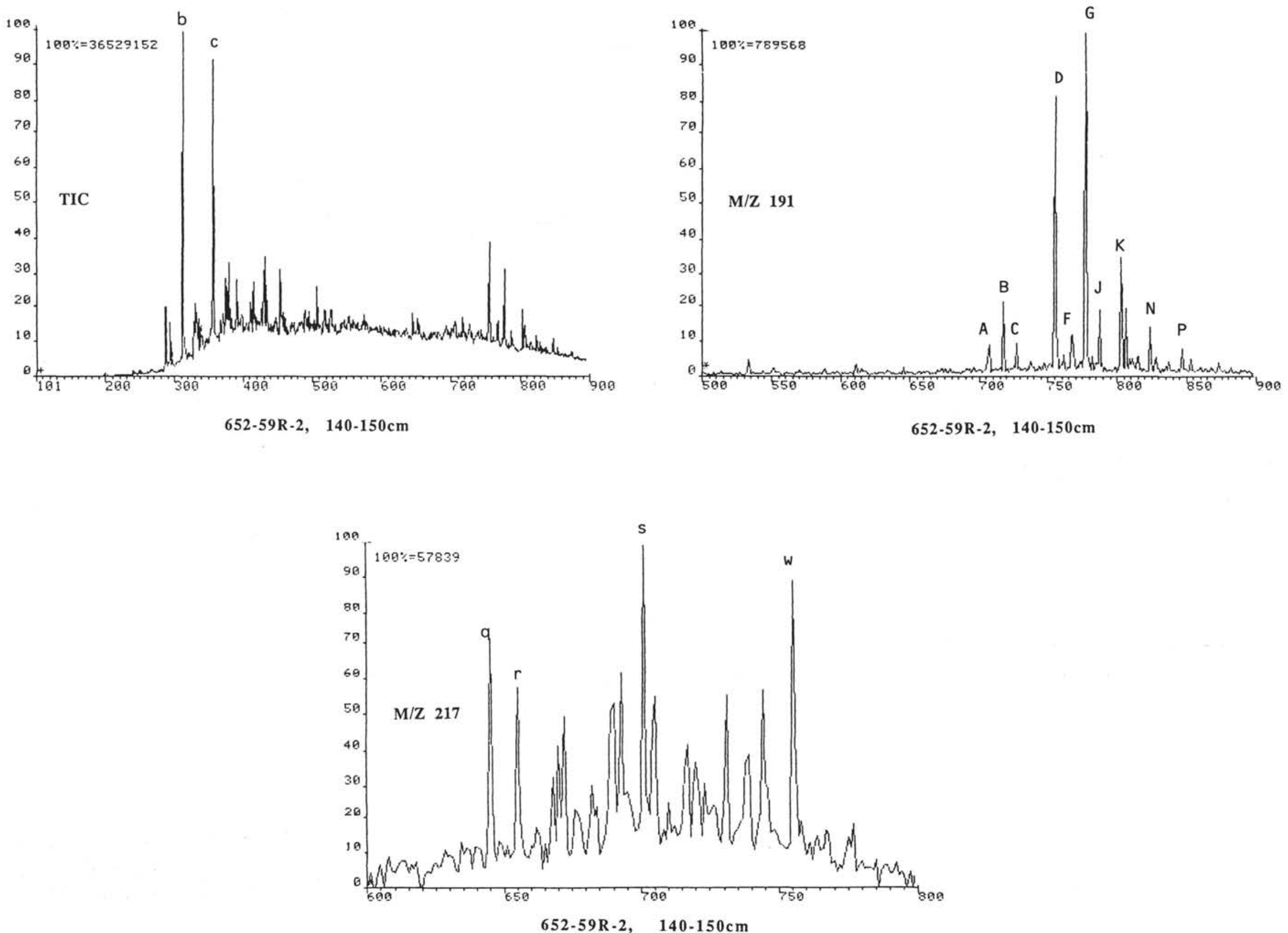

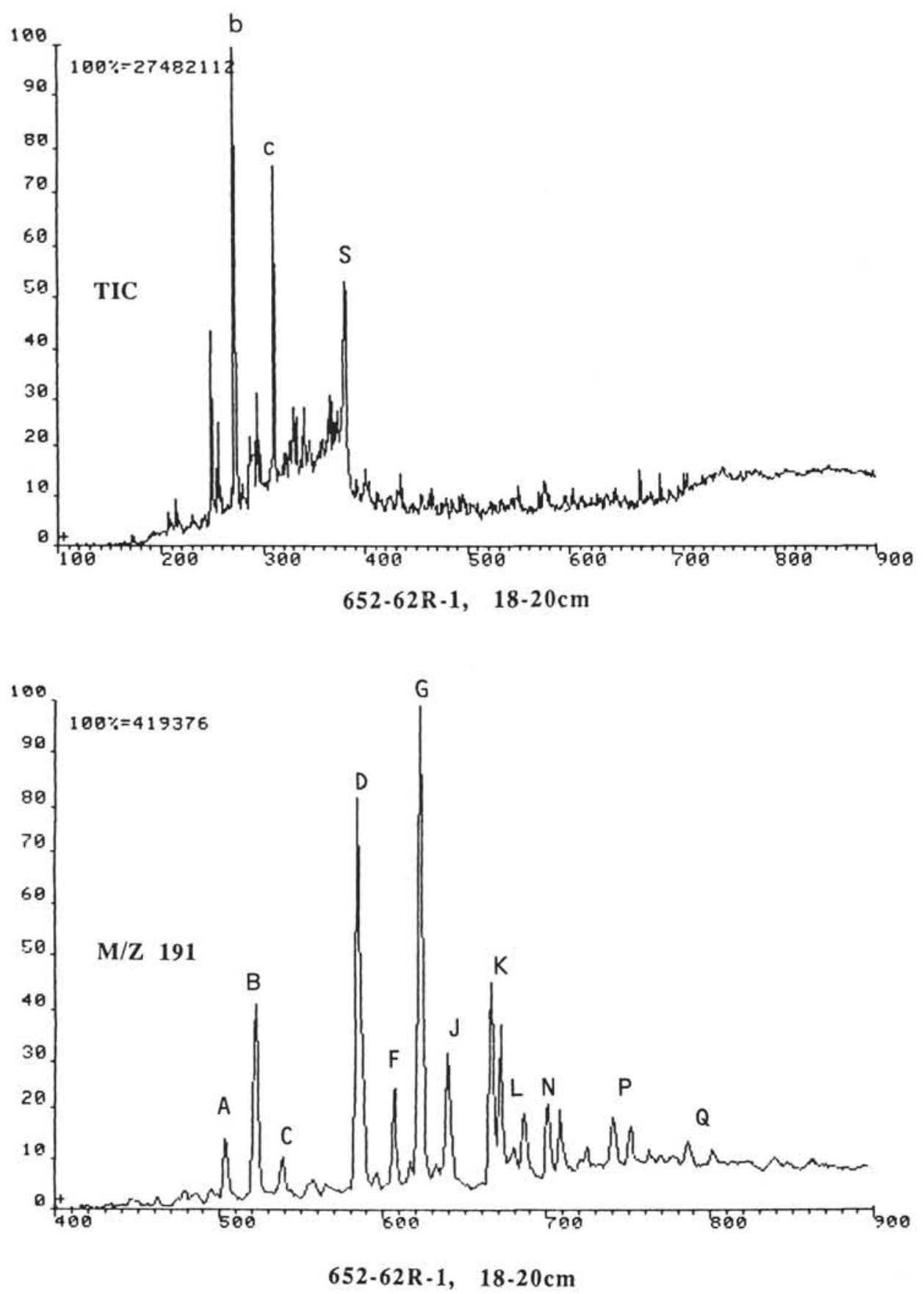

Figure 14. TIC and M/Z 191 of the branched and cyclic fraction of Sample 107-652A-62R-1, 18-20 cm. The steranes were too low in concentration to be explored by GC-MS. For identification of the assigned explored peaks refer to Table 6 .

cur mainly as unsaturated compounds (i.e., as diasterenes and spirosterenes). Only minor contributions of steranes are observed. The diasterene distribution of Sample 107-654A-38R-1, 126-132 $\mathrm{cm}$, is given in Figure 17C, which shows four major peaks. The two most prominent peaks are 20R diacholest-13(17)-ene and 20R 24-ethyldiacholest-13(17)-ene, the latter being by far the most prominent peak. The diagenetically produced $20 \mathrm{~S}$ isomers are fairly low in concentration and indicate a very low maturity of these samples (ratio $20 \mathrm{~S} / 20 \mathrm{~S}+20 \mathrm{R}$ for diacholest-13(17)ene is 0.34 ). Spirosterenes are observed in a distribution pattern that is different from the pattern of diasterene occurrence. Here the major compound is found at $\mathrm{C}_{27}$ with only minor contribu- tions of $\mathrm{C}_{29}$ (Fig. 17D). The isomerization of $20 \mathrm{R}-20 \mathrm{~S}$ is in the very early stage $(20 S / 20 S+20 R=0.08)$. Steranes are observed as trace constituents, and the $\mathrm{C}_{29}$ compounds are again slightly more abundant than the $\mathrm{C}_{27}$ molecules (Fig. 17E).

Diasterenes in Sample 107-654A-38R-2, 33-39 cm (Fig. 18C), are clearly dominated by the $20 \mathrm{~S}$ and $20 \mathrm{R}$ isomers of diacholest13(21)-ene: the ratio of $20 \mathrm{~S} / 20 \mathrm{~S}+20 \mathrm{R}$ for $\mathrm{C}_{27}$ is 0.48 and slightly higher than in Sample 107-654A-38R-1, 126-132 cm (Fig. 17C). The same unusual selectivity is observed in the sterane distribution that is predominated by the $5 \alpha, 14 \alpha, 17 \alpha$-cholestane isomer. Virtually no traces of steranes of higher molecular weight were observed (Fig. 18D). The spirosterenes of this sample are 

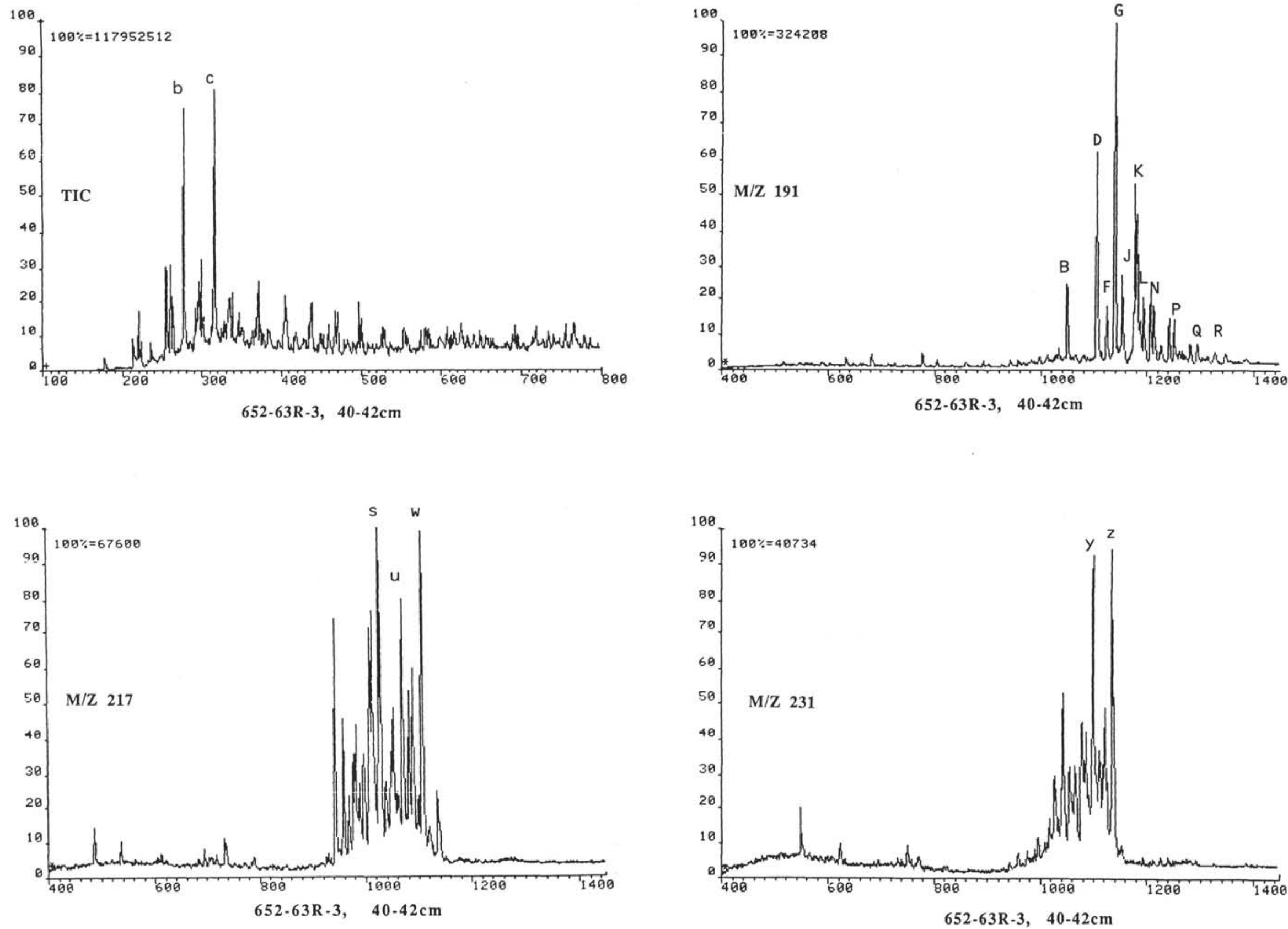

Figure 15, TIC, M/Z 191, M/Z 217, and M/Z 231 (diagnostic ion for the 4-methylsteranes) of the branched and cyclic fraction of Sample 107-652A-63R-3, 40-42 cm. For identification of the assigned peaks refer to Table 6. 

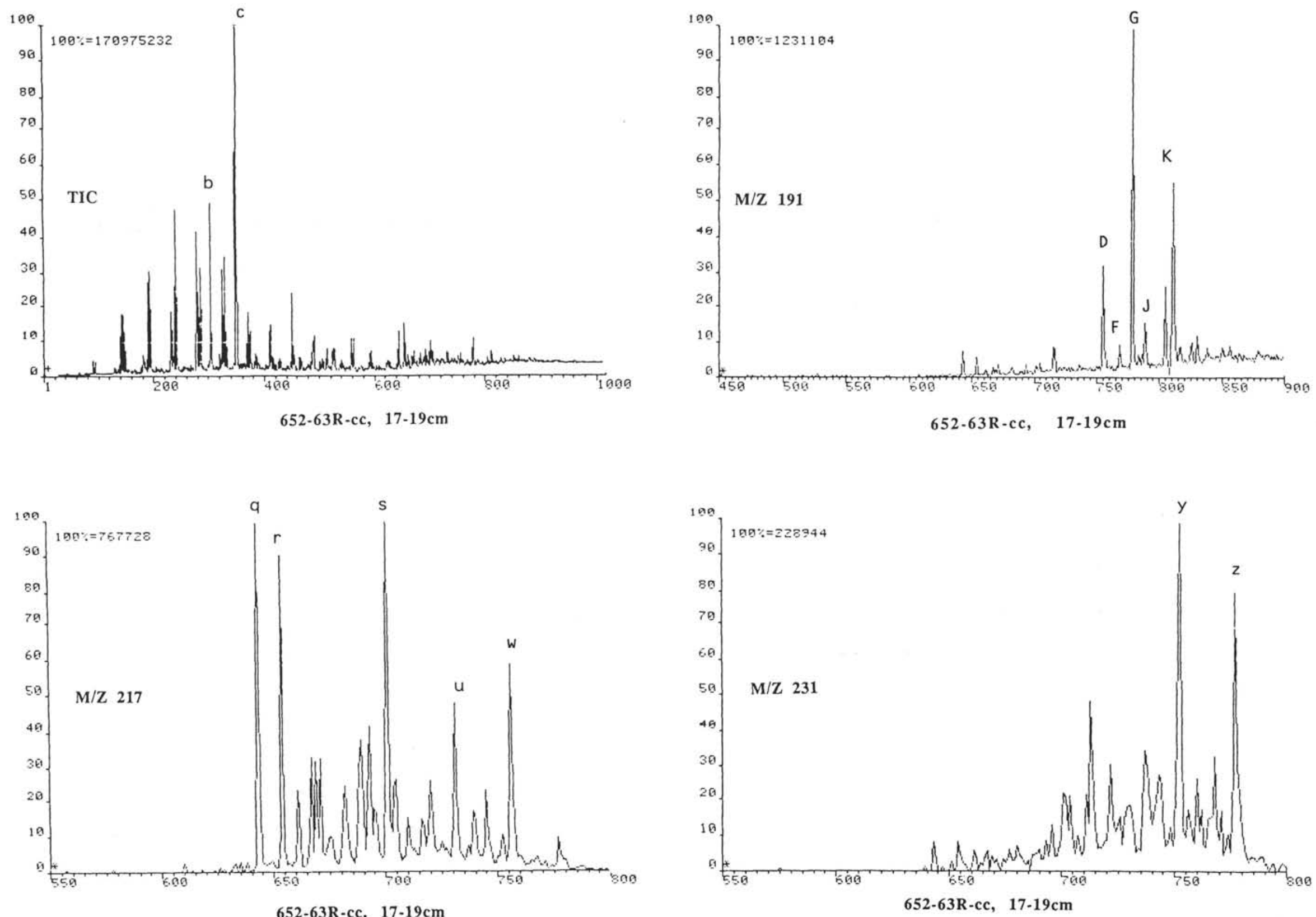

Figure 16. TIC, M/Z 191, M/Z 217, and M/Z 231 of the branched and cyclic fraction of Sample 107-652A-63R-CC, 17-19 cm. For identification of the assigned peaks refer to Table 6 . 
very abundant relative to the other steroid hydrocarbons, and the major peak is the 20R 24-ethylspirosterene (Fig. 18C). The distribution pattern of spirosterenes is rather complex compared with the diasterenes. At least two isomers of $\mathrm{C} 27, \mathrm{C} 28$, and $\mathrm{C} 29$, respectively, were identifiable. Of these, 4-methylspirosterenes may considerably complicate the distribution of spirosterenes. While a $\mathrm{C}_{29}$ compound is the dominant constituent, $\mathrm{C}_{28}$ and $\mathrm{C}_{27}$ spirosterenes are equally abundant; this distribution contrasts the character of diasterene and sterane distributions. Spirosterenes and diasterenes are intermediate products in the maturation of steroids (Mackenzie et al., 1982). The fact that the major isomer at each $\mathrm{C}$-atom is the $20 \mathrm{R}$ spirosterene indicates incomplete isomerization and suggests thermal immaturity of the sample. The ratio $20 \mathrm{~S} / 20 \mathrm{~S}+20 \mathrm{R}$ is 0.39 for Sample 107-654A-38R-2, 33-39 cm.

The internal distribution of steroid hydrocarbons in the samples of Hole 654A are presented in Figures 19 and 20. There is an obvious problem concerning the sources of the steroid hydrocarbons in these two samples. It is generally assumed that a certain amount of sterols with a particular distribution of $\mathrm{C}_{27}$ $\mathrm{C}_{29}$ compounds (depending on the biological precursor) is the source of the sedimentary steroid hydrocarbons. In the case of the two sediment samples from Hole 654A, the distribution patterns of individual steroid hydrocarbon fractions are not comparable. The diagenetic transformation of sterols via sterenes, diasterenes, and spirosterenes to steranes should not disturb the internal distribution pattern of the precursor sterols. A possible explanation for such different distributions within each fraction of steroid hydrocarbons may be that different sources supplied the sterol material. A marine source consisting of abundant $\Delta^{7}$ sterenes, a major constituent of seawater steroids that has not yet been found in sediments (Brassell et al., 1982), could give rise to abundant $\mathrm{C}_{27}$ spirosterenes (Peakman et al., 1982). An additional terrigenous source with abundant $\mathrm{C}_{29}$ sterols affected by clay mineral catalysis may be responsible for the formation of diasterenes with a dominance of 24-ethyldiacholest-13(17)ene. The subsequent reduction of these two groups of alcenes could then yield the observed distribution of steranes. However, different and yet unknown mechanisms have been responsible for the distribution of steroid hydrocarbons in Sample 107-654A38R-2, 33-39 cm.

In three samples of Hole $652 \mathrm{~A}$ that were comparable on the basis of their hopane distributions (107-652A-58R-5, 67-69 cm (Fig. 12); 107-652A-59R-2, 140-150 cm (Fig. 13); and 107-652A$62 \mathrm{R}-1,18-20 \mathrm{~cm}$ (Fig. 14)), the steroid hydrocarbons are only minor contributors and we observed no unsaturated steroid hydrocarbons. The higher maturity of samples from Hole $652 \mathrm{~A}$, when compared with those of Hole $654 \mathrm{~A}$, is reflected in the degree of isomerization of steranes. In detail, the $\beta \beta / \beta \beta+\alpha \alpha 24-$ ethylsterane isomerization ratio is around 0.40 for three of the samples of Hole $652 \mathrm{~A}$. The $20 \mathrm{~S} / 20 \mathrm{~S}+20 \mathrm{R}$ isomerization ratio of 24-ethylsterane is varying in a range between 0.36 and 0.39 . Based on the sterane data, Sample 107-652A-63R-3, 40-42 cm (Fig. 15), also shows some similarities to the group of shallower samples listed above. All samples expose a more or less equal abundance of cholestane and 24-ethylcholestane. In addition, an equivalent series of diasteranes is present and parallels the distribution of the steranes. For this reason, a similar source for the diasteranes and the steranes appears likely. Such a similarity in the distribution contrasts the observations from Hole 654A. Sample 107-652A-63R-CC, 17-19 cm (Fig. 16), is an exception from the similar group of sediments because it is clearly dominated by cholestane isomers. The maturity index $20 \mathrm{~S} / 20 \mathrm{~S}+$ $20 \mathrm{R}(0.43)$ is higher, while $\beta \beta / \beta \beta+\alpha \alpha(0.25)$ for the 24-ethylcholestane is considerably lower than in the other samples of Hole $652 \mathrm{~A}$. This is surprising, as it is the deepest sample analyzed from this hole. The reason for the difference may be re- lated to a differing isomerization behavior of the steranes due to the high organic carbon content and the different nature of organic matter. The inverse relationship between organic carbon and maturity indices is shown in Figures 21A and 21B. A drastic increase in organic carbon below 590 mbsf in Hole $652 \mathrm{~A}$ is accompanied by a change to a presumably anoxic paleoenvironment. Hopane and sterane isomerization ratios change to lower maturity ratios simultaneously and substantiate the assumption that organic matter abundance or type influence molecular maturation parameters besides thermal catagenesis.

Anomalous isomerization of steranes and hopanes has previously been observed in hypersaline paleoenvironments, where the ratios of $\beta \beta / \alpha \alpha+\beta \beta$ for the steranes and $20 \mathrm{~S} / 20 \mathrm{~S}+20 \mathrm{R}$ for the hopanes change to significantly higher values (ten Haven et al., 1986). The complete isomerization data for the steranes and the hopanes from Hole $652 \mathrm{~A}$ are summarized in Table 5.

4-methylsteranes were detected in significant relative quantities in the organic-carbon-rich Samples 107-652A-63R-3, 40-42 $\mathrm{cm}$, and 107-652A-63R-CC, $17-19 \mathrm{~cm}$, as shown in Figures 15 and 16 (Table 6). The spectra of the oil-shale interval are dominated by two C-30 isomers that could not be conclusively identified. It is probable, however, that these peaks reflect isomers of dinosterane $(4,23,24$-rimethylcholestane). Even though microscopic investigation failed to provide evidence for the contribution of dinoflagellates to these strata, we believe that dinoflagellate debris or bacteria are likely candidates for the accumulation of millimeter-thick organic layers. In other samples that were presumably deposited under oxic conditions, only trace amounts of 4-methylsteranes were observed. These traces are again predominated by $\mathrm{C} 30$ compounds, indicating a potentially continuous contribution of specific types of organisms (notably dinoflagellates) with varying degrees of preservation depending upon the paleoenvironmental conditions.

\section{Microscopic Investigation}

We hoped to substantiate our notion that dinoflagellates or related nonskeletal algae contributed to the laminated oil shale interval encountered in Core 107-652A-64R, which appears to be an excellent and mature hydrocarbon source rock (Figs. 22 and 23). We had assumed that algae similar to Botryococcus braunii may have been the dominant input, because pyrolysis results and general facies supported a brackish paleoenvironment (Site 652; Kastens, Mascle, et al., 1987). We failed to recognize intact algal cells under the microscope, however. When excited with UV light, the millimetric laminations fluoresce strongly and in a yellow/orange color. The laminations persist to the scale of fractions of millimeters (Figs. 23A and 23B), and their intimate relationship with clastic grains lead us to believe that we are dealing with stromatolithic bacterial mats interspersed in a clastic-dominated fluvial or lacustrine environment, which must have been in relatively close proximity to saline waters.

\section{CONCLUSIONS}

The evolution of the Sardinian margin was accompanied by deposition of organic matter in sediments of considerable facies diversity and in paleoenvironmental difference between the Messinian of Holes 652A and 654A. Organic matter composition in these two environments appears to have been derived from similar sources, and a general continental provenance is likely for the Messinian background organic matter. The sediments of Hole 654A appear somewhat more lipid-rich, however, and one may argue the assumption that continental organic carbon constitutes the only source. The Tortonian sediments analyzed from Hole 654A are surprisingly low in lipid-rich material, and in spite of definitely marine depositional conditions, the organic content appears to be continent-derived. Pliocene and Pleistocene hemipelagic calcareous oozes and volcanogenic sediments 

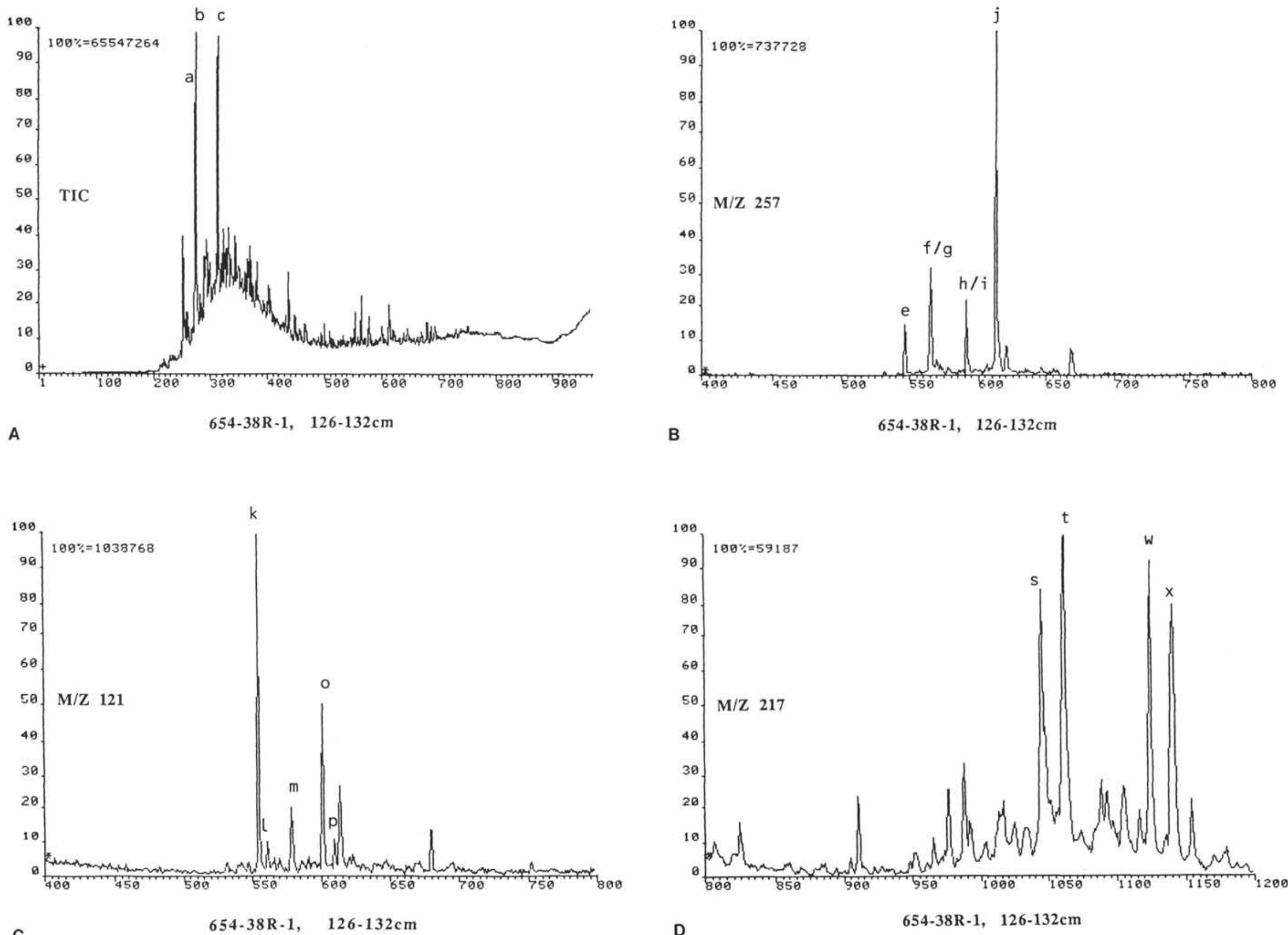


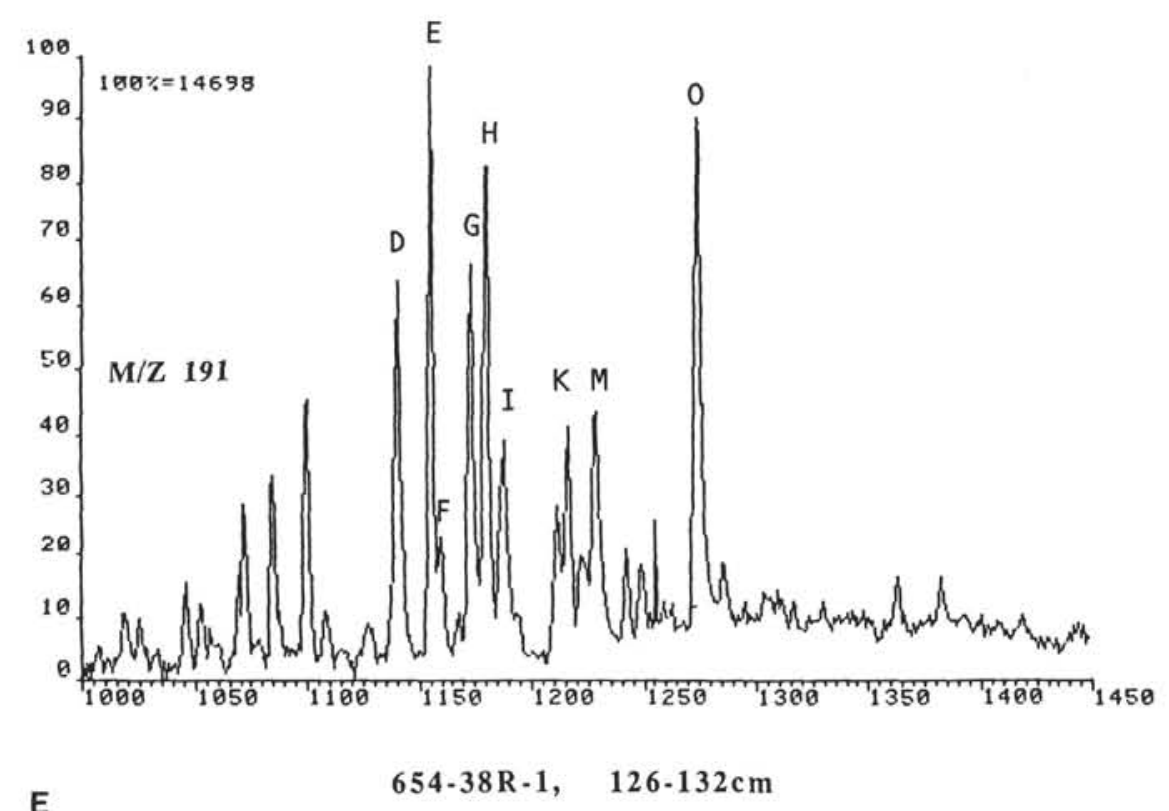

Figure 17. TIC, M/Z 257 (diagnostic ion for the diasterenes), M/Z 121 (diagnostic ion for the spirosterenes), M/Z 217, and M/Z 191 of the branched and cyclic fraction of Sample 107-654A-38R$1,126-132 \mathrm{~cm}$. For identification of the assigned peaks refer to Table 6. 

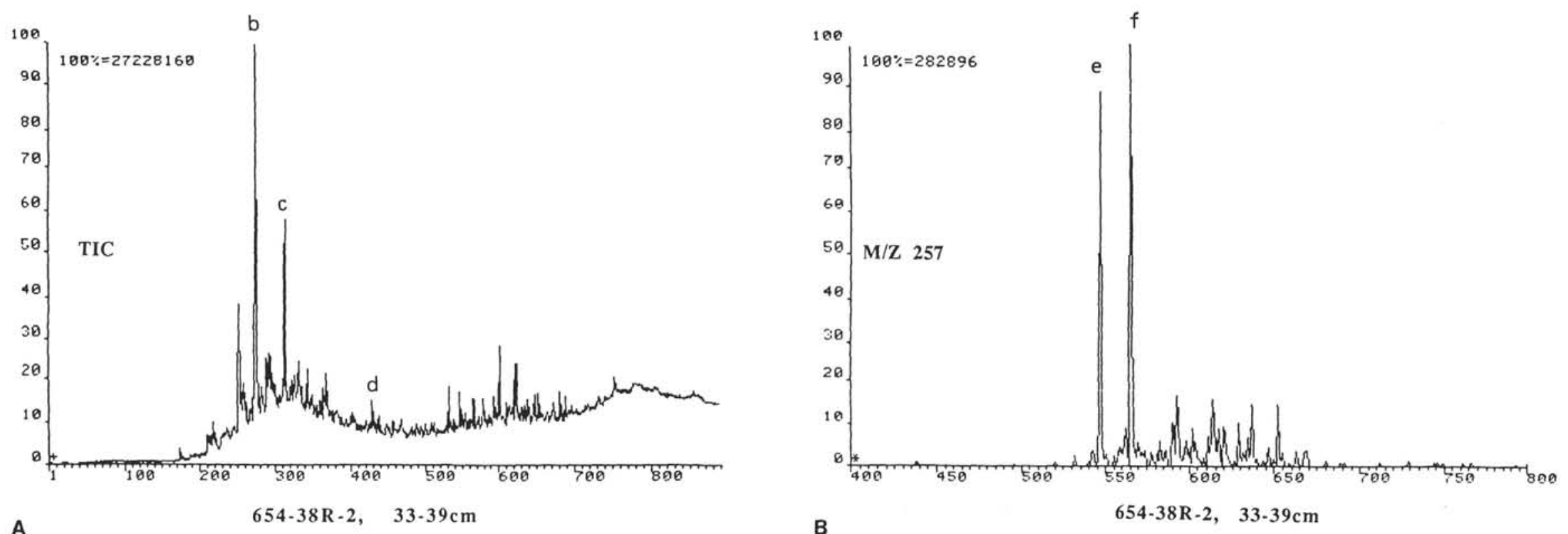

A

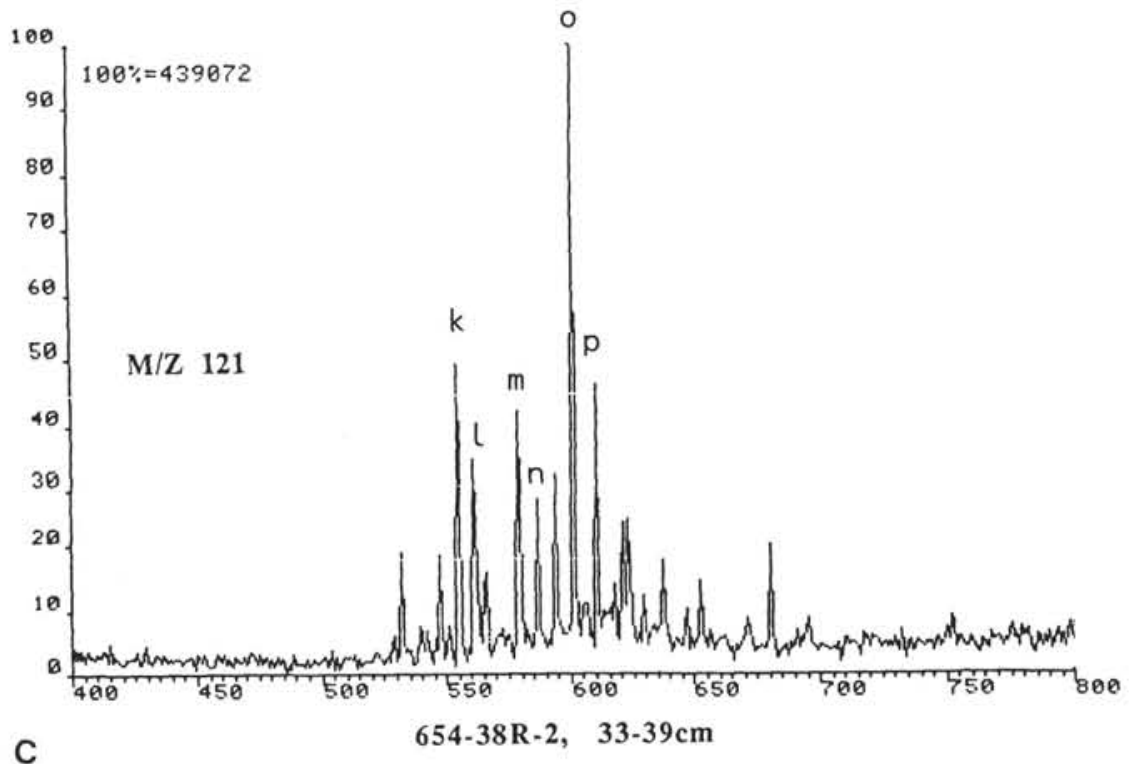

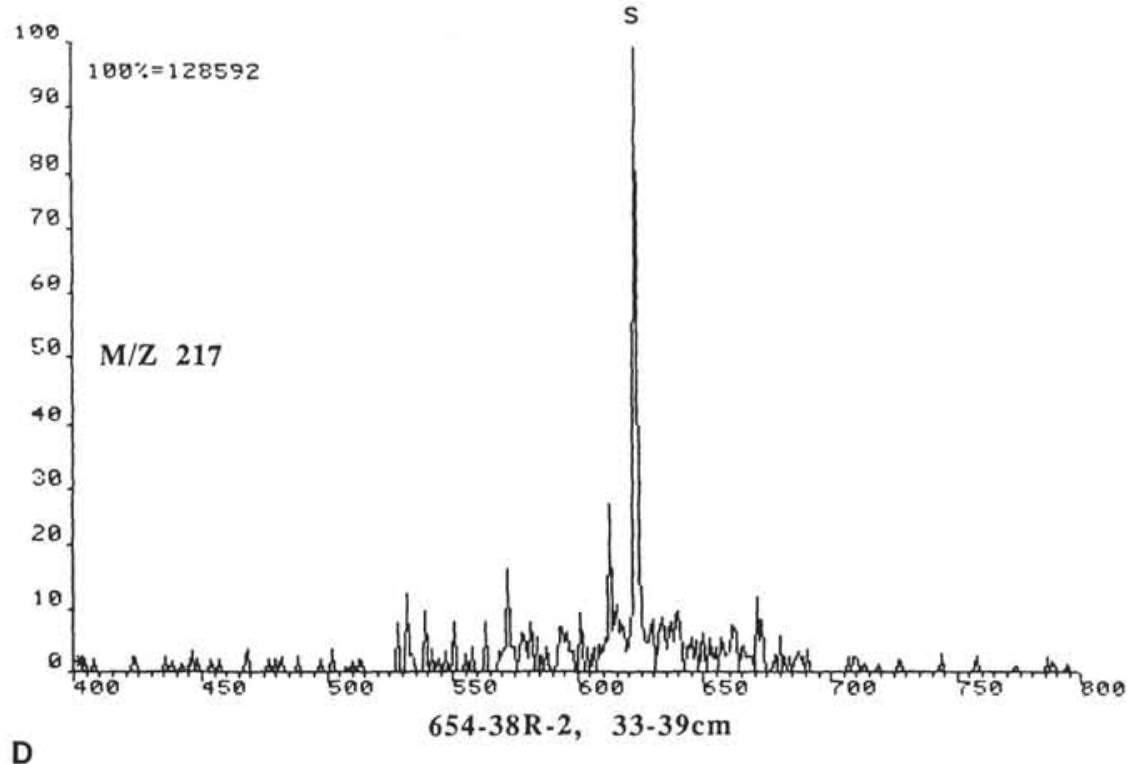




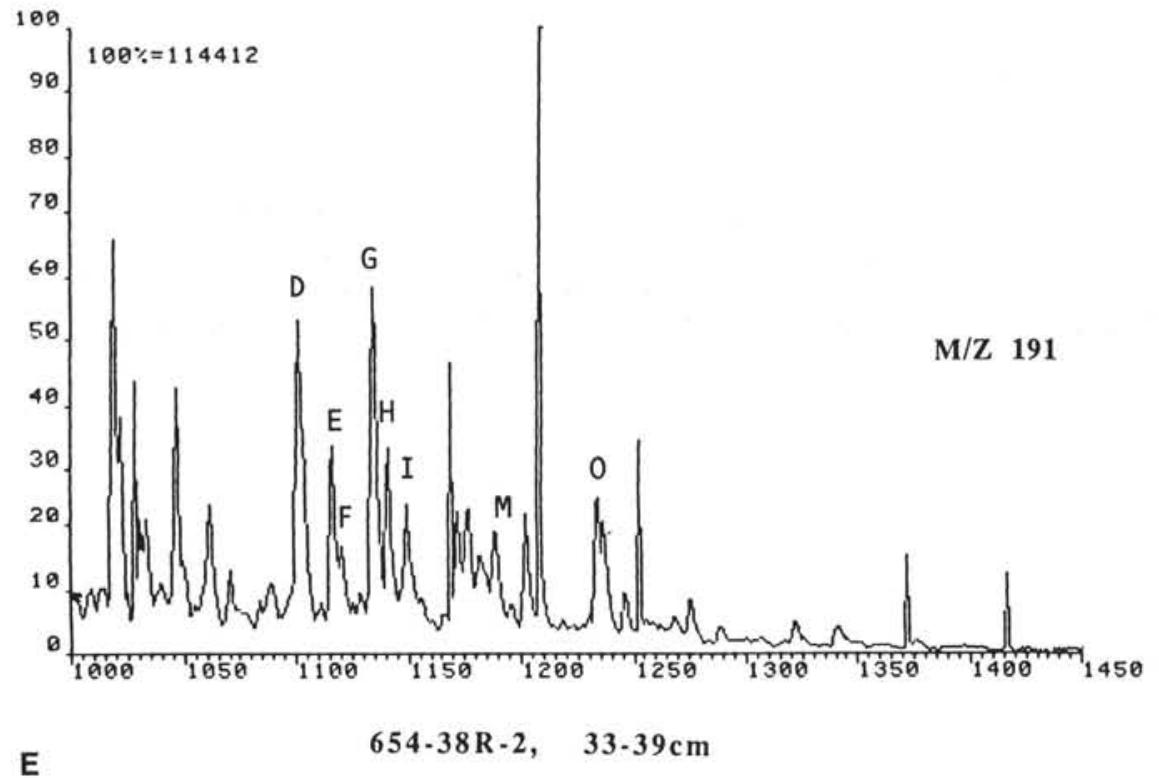

Figure 18. TIC, M/Z 257, M/Z 121, M/Z 217, and M/Z 191 of the branched and cyclic fraction of Sample 107-654A-38R-2, 33-39 cm. For identification of the assigned peaks to Table 6. 
Table 5. Molecular maturation indexes for five samples from the Messinian section of Hole $652 \mathrm{~A}$.

\begin{tabular}{lccccc}
\hline \multicolumn{1}{c}{ Sample } & $58 \mathrm{R}-5$ & $59 \mathrm{R}-2$ & $62 \mathrm{R}-1$ & $63 \mathrm{R}-3$ & $63 \mathrm{R}, \mathrm{CC}$ \\
\hline $\begin{array}{l}\text { 24-ethylcholestane } \\
\beta \beta / \beta \beta+\alpha \alpha\end{array}$ & 0.35 & 0.41 & 0.41 & 0.35 & 0.25 \\
$\begin{array}{l}\text { 24-ethylcholestane } \\
\text { 20S } / 20 \mathrm{~S}+20 \mathrm{R}\end{array}$ & 0.38 & 0.36 & 0.39 & 0.43 & 0.43 \\
$\begin{array}{l}\text { homohopane }(17 \alpha, 21 \beta) \\
\text { 22S } / 22 \mathrm{~S}+22 \mathrm{R}\end{array}$ & 0.64 & 0.65 & 0.64 & 0.55 & 0.30 \\
\hline
\end{tabular}

\section{$654-38 \mathrm{R}-1, \quad 126-132 \mathrm{~cm}$}

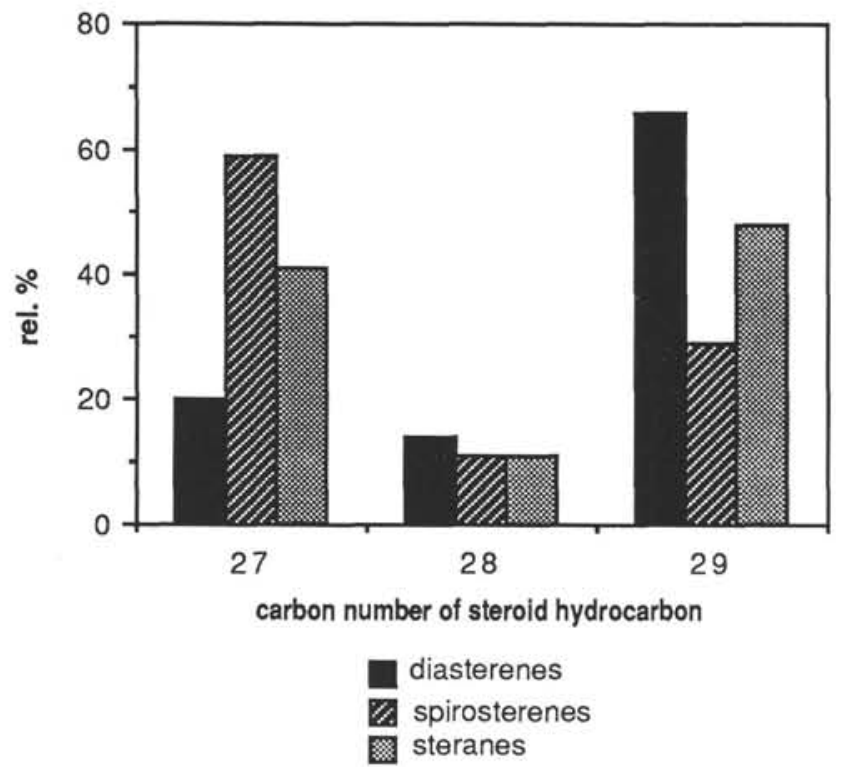

Figure 19. Carbon number distribution of diasterenes, spirosterenes, and steranes in Sample 107-654A-38R-1, 126-132 cm.

are exceedingly low in organic matter, for which we assume a residual and recycled nature.

Notable exceptions of this rather uniform organic matter character are found only in two kinds of sediments, where high organic carbon concentrations and chemical composition outline deviating biological and depositional conditions. These two environments-an oil shale in Hole $652 \mathrm{~A}$ and sapropels in the Pleistocene-have TOC concentrations of up to $11 \%$ and $6 \%$ TOC, respectively. The oil shale contains very lipid-rich organic matter of kerogen Type 1. The sapropels appear to contain organic matter from marine sources.

Maturation history of the lower and upper continental margin of Sardinia has been different for the two sites. We found evidence of a higher degree of maturation at Site 652, where higher heat flow must have prevailed in the Messinian. The Messinian sediments at this site are thermally mature and, in the case of high organic carbon content, are a hydrocarbon source. Sediments at Site 654, in contrast, are thermally immature. Hopane distributions show that lipids from a mature source coexist with immature lipids in samples of Messinian age in evaporitic facies and imply that migration of hydrocarbons from a deeper, mature source has taken place.

Alkane and cycloalkane distributions in Hole 654A show strong odd-over-even predominance in carbon atom numbers, which is a characteristic inherited from the biological precursor
$654-38 \mathrm{R}-2, \quad 33-39 \mathrm{~cm}$

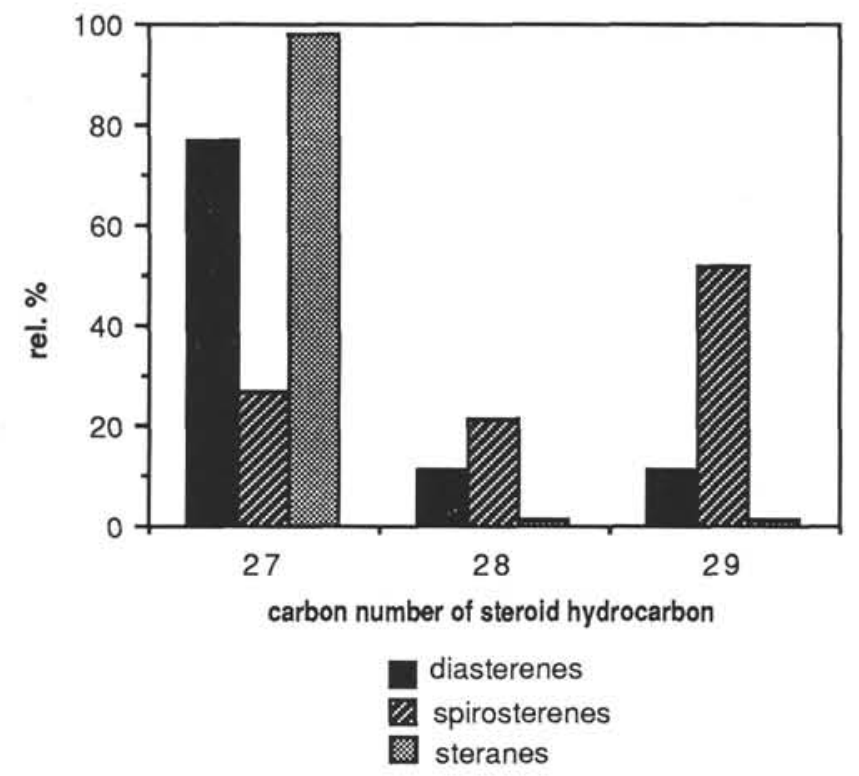

Figure 20. Carbon number distribution of diasterenes, spirosterenes, and steranes in Sample 107-654A-38R-2, 33-39 cm.

molecules. This fact and the presence of a variety of unsaturated compounds that include spirosterenes, diasterenes, and hopenes underscore the thermal immaturity of lipids, and of organic matter in general.

Maturation trends based on hopane and sterane distribution with depth appear to be in part inverted in the section of Hole $652 \mathrm{~A}$. A potential explanation for the unusual distribution of molecular maturity indicators in the oil-shale interval at Core 107-652A-64R when compared with adjacent organic-lean sediments is that the oil shale was probably deposited in a different type of environment (i.e., not hypersaline). The maturity indices within this interval are the correct ones, whereas those from the adjacent organic-lean strata are modified by anomalous diagenetic transformation of $\Delta^{7}$-sterenes to $14 \beta(\mathrm{H}), 17 \beta(\mathrm{H})$ steranes and the direct reduction of extended hopenes to hopanes and not via isomerization of the $22 \mathrm{R} \beta \beta$-hopanes (Ten Haven et al., 1986).

The discovery in the evaporitic sediments of Messinian age of 3-(4,8,12-trimethyltridecyl)-thiophene, 2,6,10-trimethyl-7-(3methyl-butyl)-dodecane, and of gammacerane, the occurrence of which are believed to be restricted to evaporitic depositional environments, corroborates their usefulness as paleoenvironmental indicators for evaporitic settings.

The observed variety of steroid biomarker molecules is difficult to reconcile with a single biological source. In all likelihood, multiple sources coexisted that provided varieties of initial sterol compounds to the sediments. Postdepositional modifications of steroid molecules by acidic clay catalysis, isomerization, and rearrangement reactions in a hypersaline environment may have further complicated the distribution of steroids.

\section{ACKNOWLEDGMENTS}

Laboratory assistance of H.-H. Richnow, U. Kruse, and T. Frank is gratefully acknowledged, as well as technical support by the Ocean Drilling Program. We appreciate the thorough reviews of Drs. K. Kastens, J. Mascle, F. McCoy, J. Jasper, and an anonymous reviewer. Financial support for this study was received from the Deutsche Forschungsgemeinschaft (grant DFG De 74/36-1) and by USSAC. 

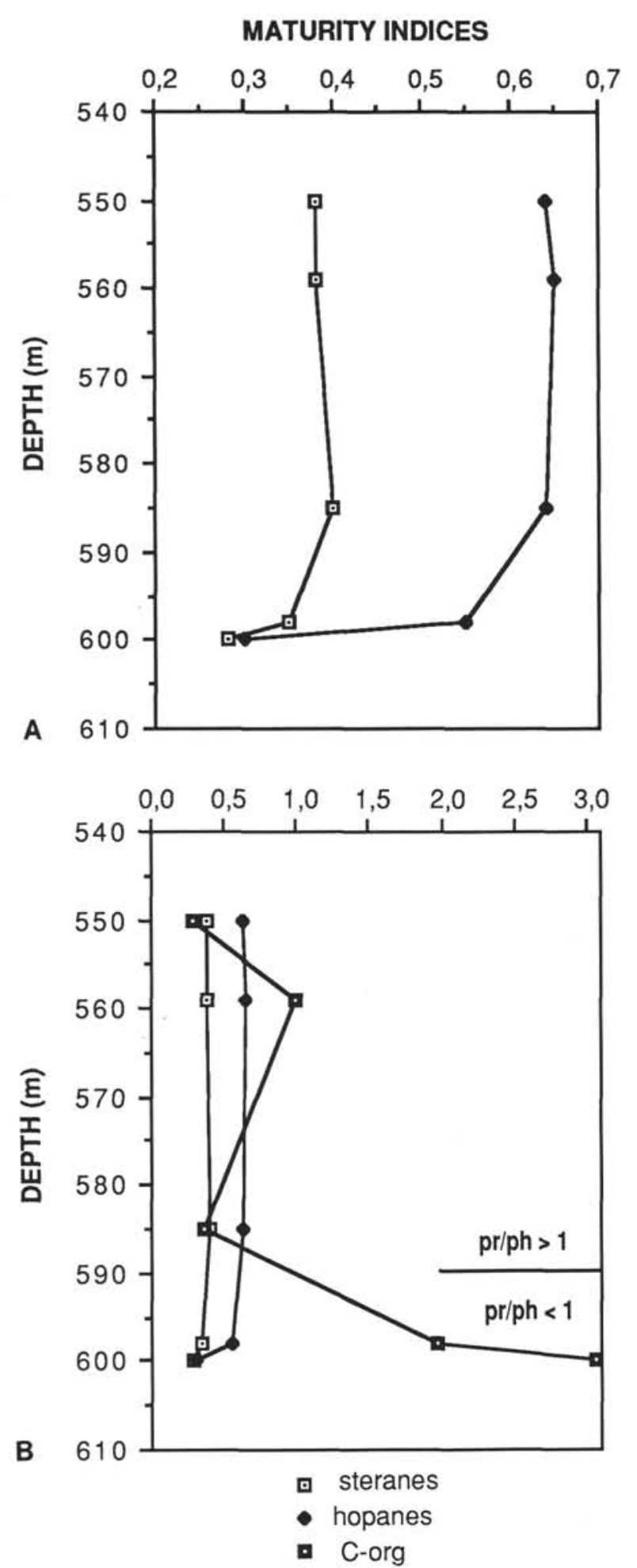

Figure 21. A. Maturity indices $\beta \beta / \beta \beta+\alpha \alpha$ for 24-ethylcholestane and $22 \mathrm{~S} / 22 \mathrm{~S}+22 \mathrm{R}$ for homohopane $(17 \alpha, 21 \beta)$ in Hole 652A of Leg 107 in the Tyrrhenian Sea. B. Maturity indices of A plotted with depth in combination with the TOC of the respective sediments. The boundary of $\mathrm{pr} /$ $\mathrm{ph}>1 \mathrm{vs} . \mathrm{pr} / \mathrm{ph}<1$ is shown at a depth of around $590 \mathrm{~m}$.

\section{REFERENCES}

Bray, E. E., and Evans, E. D., 1961. Distribution of $n$-paraffins as a clue to recognition of source beds. Geochim. Cosmochim. Acta, 22: 2-15.

Brassell, S. C., McEvoy, J., Hoffmann, C. F., Lamb, N. A., Peakman, T. M., and Maxwell, J. R., 1984. Isomerization, rearrangement and aromatization of steroids in distinguishing early stages of diagenesis. Org. Geochem., 6:11- 23.

Brassell, S. C., Lewis, C. A., de Leeuw, J. W., de Lange, F., and Sinninghe Damste, J. S., 1986. Isoprenoid thiophenes: novel products of sediment diagenesis? Nature, 320:160-162.

Broquet, P., Mascle, G., and Monier, M., 1984. La formation a Tripolis du bassin de Caltanissetta (Sicile). Rév. de Géologie Dynamique et de Géographie Physique, 25:87-98.

Connan, J., Bouroullec, J., Dessort, D., and Albrecht, P., 1986. The microbial input in carbonate-anhydrite facies of a sabkha paleoenvironment from Guatemala: a molecular approach. Org. Geochem., 10:29-50.

Connan, J., and Cassou, A. M., 1980. Properties of gases and petroleum liquids derived from terrestrial kerogen at various maturation levels. Geochim. Cosmochim. Acta, 44:1-23.

Didyk, B. M., Simoneit, B.R.T., Brassell, S. C., and Eglinton, G., 1978. Organic geochemical indicators of paleoenvironmental conditions of sedimentation. Nature, 272:216-222.

Espitalié, J., Deroo, G., and Marquis, F., 1985. La pyrolyse Rock-Eval et ses applications, Part 1. Revue de l'Institut Français du Petrole, 40:563-579.

Kastens, K. A., Mascle, J., et al., 1987. Proc. ODP, Init. Repts., 107: College Station, TX (Ocean Drilling Program).

Katz, B. J., 1983. Limitations for "Rock Eval" pyrolysis for typing organic matter. Org. Geochem. 4:195-199.

Klok, J., Cox, C., Baas, M., de Leeuw, J. W., and Schenck, P. A., 1984. Carbohydrates in recent marine environments-II. Occurrence and fate of carbohydrates in a recent stromatolithic deposit: Solar Lake, Israel. Org. Geochem., 7:101-109.

Mackenzie, A. S., Brassell, S. C., Eglinton, G., and Maxwell, J. R., 1982. Chemical fossils: the geochemical fate of steroids. Science, 217:491-504.

Peakman, T. M., Lamb, N. A., and Maxwell, J. R., 1984. Naturally occurring spiro steroid hydrocarbons. Tet. Lett., 25:349-352.

Powell, T. G., and McKirdy, D. M., 1973. Relationship between ratio of pristane to phytane, crude oil composition, and geological environment in Australia. Nature Phys. Sci., 243:37-39.

Robson, J. N., and Rowland, S. J., 1986. Identification of novel widely distributed sedimentary acyclic sesterpenoids. Nature, 324:561-563.

Rubinstein, I., Sieskind, O., and Albrecht, P., 1975. Rearranged sterenes in a shale: occurrence and simulated formation. J. Chem. Soc. Perkin I., 1833-1836.

Rullkötter, J., Mukhopadhyay, P. K., and Welte, D. H., 1984. Geochemistry and petrography of organic matter in sediments from Hole 530A, Angola Basin, and Hole 532, Walvis Ridge. In Hay, W. W., Sibuet, J. P., et al., Init. Repts. DSDP, 75: Washington (U.S. Govt. Printing Office), 1069-1087.

Ryan, W.B.F., Hsü, K. J., et al., 1973. Init. Repts. DSDP, 13: Washington (U.S. Govt. Printing Office).

Ten Haven, H. L., de Leeuw, J. W., Sinninghe Damste, J. S., Schenck, P. A., Palmer, S. E., and Zumberge, J. E., in press. The application of biological markers in the recognition of paleo-hypersaline environments. In Kelts, K., Fleet, A., and Talbot, M. (Eds.), Lacustrine Petroleum Source Rocks.

Ten Haven, H. L., de Leeuw, J. W., Peakman, T. M., and Maxwell, J. R., 1986. Anomalies in steroid and hopanoid maturity indices. Geochim. Cosmochim. Acta, 50:853-855.

van Krevelen, D. W., 1961. Coal: Amsterdam (Elsevier).

Yon, D. A., Maxwell, J. R., and Ryback, G., 1982. 2,6,10-trimethyl7-(3 methylbutyl)-dodecane, a novel sedimentary biological marker compound. Tet. Lett., 23:2143-2146.

Date of initial receipt: 28 January 1988

Date of acceptance: 2 December 1988

Ms 107B-116 
Table 6. List of compounds identified by GC-MS and marked on Figures 12-18.

\author{
a 2,6,10-trimethyl-7-(3-methylbutyl)-dodecane \\ b pristane \\ c phytane \\ d 3-(4,8,12-trimethyltridecyl)-thiophene \\ $\mathrm{S}$ elemental sulfur $\mathrm{S}_{8}$
}

Tetracyclic molecules:

e $20 \mathrm{~S}$ diacholest-13(17)-ene

f 20R diacholest-13(17)-ene

g 20S 24-methyldiacholest-13(17)-ene

h 20R 24-methyldiacholest-13(17)-ene

i 20S 24-ethyldiacholest-13(17)-ene

j 20R 24-ethyldiacholest-13(17)-ene

k 20R spirosterene

i $20 \mathrm{~S}$ spirosterene

m 20R 24-methylspirosterene

n 20S 24-methylspirosterene

o 20R 24-ethylspirosterene

p 20S 24-ethylspirosterene

q 20 S diacholestane

$r$ 20R diacholestane

s $5 \alpha(\mathrm{H}), 14 \alpha(\mathrm{H}), 17 \alpha(\mathrm{H})$-cholestane

t $5 \beta(\mathrm{H}), 14 \alpha(\mathrm{H}), 17 \alpha(\mathrm{H})$-cholestane

u $5 \alpha(\mathrm{H}), 14 \alpha(\mathrm{H}), 17 \alpha(\mathrm{H})$-24-methylcholestane

v $5 \beta(\mathrm{H}), 14 \alpha(\mathrm{H}), 17 \alpha(\mathrm{H})$-24-methylcholestane

w $5 \alpha(\mathrm{H}), 14 \alpha(\mathrm{H}), 17 \alpha(\mathrm{H})$-24-ethylcholestane

x $5 \beta(\mathrm{H}), 14 \alpha(\mathrm{H}), 17 \alpha(\mathrm{H})$-24-ethylcholestane

y 4-methylsterane (C30)

z 4-methylsterane (C30)
Pentacyclic molecules:

A $18 \alpha(\mathrm{H})$-trisnorhopane $\mathrm{T}_{\text {s }}$

B $17 \alpha(\mathrm{H})$-trisnorhopane $T_{m}$

C $17 \alpha(\mathrm{H}), 21 \beta(\mathrm{H})$ bisnorhopane

D $17 \alpha(\mathrm{H}), 21 \beta(\mathrm{H})$-30-norhopane

E hop-17(21)-ene

F 30-normoretane

G $17 \alpha(\mathrm{H}), 21 \beta(\mathrm{H})$-hopane

$\mathrm{H}$ neohop-13(18)-ene

I $17 \beta(\mathrm{H}), 21 \beta(\mathrm{H})-30$-norhopane

$\mathrm{J}$ moretane

K 20S, R $17 \alpha(\mathrm{H}), 21 \beta(\mathrm{H})$-homohopane

$\mathrm{L}$ homomoretane

M $17 \beta(\mathrm{H}), 21 \beta(\mathrm{H})$-hopane

N 20S, R $17 \alpha(\mathrm{H}), 21 \beta(\mathrm{H})$-bishomohopane

O $17 \beta(\mathrm{H}), 21 \beta(\mathrm{H})$-homohopane

P $20 S, \mathrm{R} 17 \alpha(\mathrm{H}), 21 \beta(\mathrm{H})$-trishomohopane

Q 20S, R $17 \alpha(\mathrm{H}), 21 \beta(\mathrm{H})$-tetrakishomohopane

R $20 \mathrm{~S}, \mathrm{R} 17 \alpha(\mathrm{H}), 21 \beta(\mathrm{H})$-pentakishomohopane 


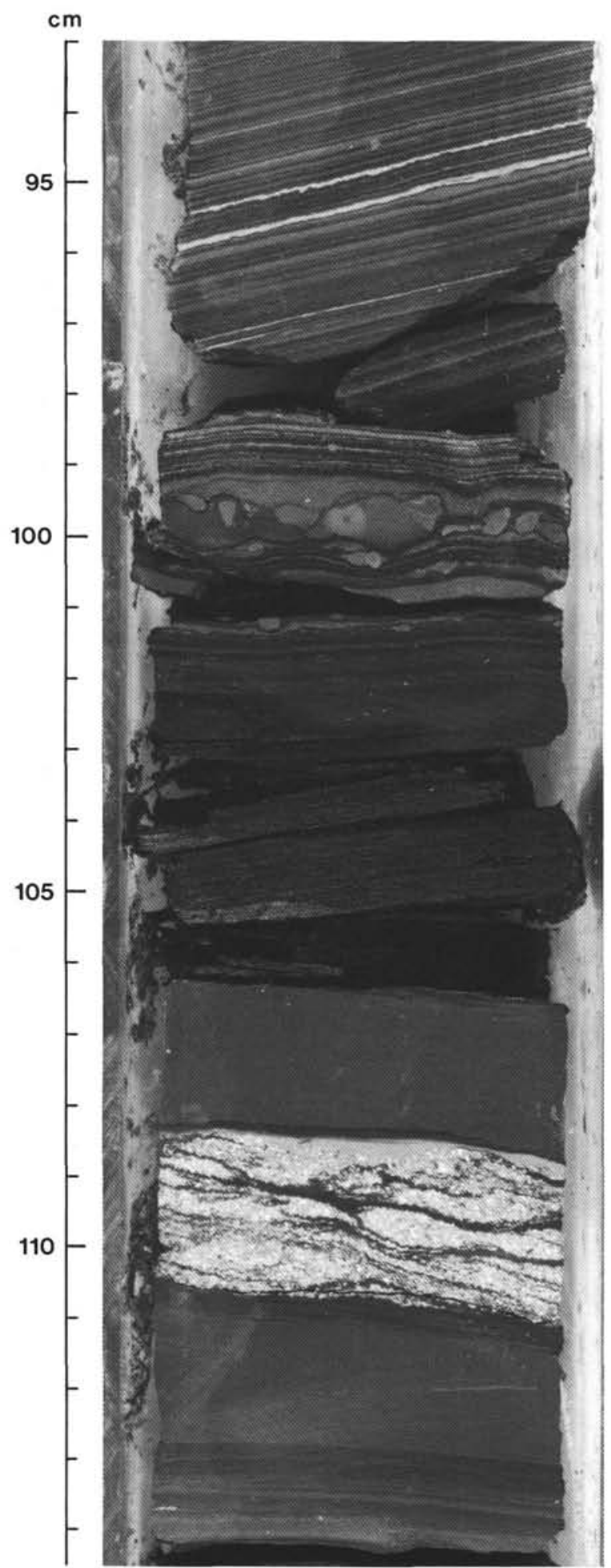

Figure 22. Close-up photograph of Section 107-652A-64R-1, 93-114 cm (605 mbsf). The figure depicts the oil shale interval of Messinian age (Interval 100-108 cm) sandwiched between clastic mudstones of brackish, fluvial, or lacustrine origin. Note the persistent laminations above and below and the incorporation of mud clasts above the finely laminated interval between 101 and $106 \mathrm{~cm}$. The white layer at $108-111 \mathrm{~cm}$ is partly composed of gypsum crystals, but dominated by flaky, amorphous material that could not be analyzed by energy dispersive $x$-ray analysis (Emeis, unpubl. data). TOC in this white layer is only about $2 \%$. Note the different scale of layer thickness in the mudstone and the oil shale. 


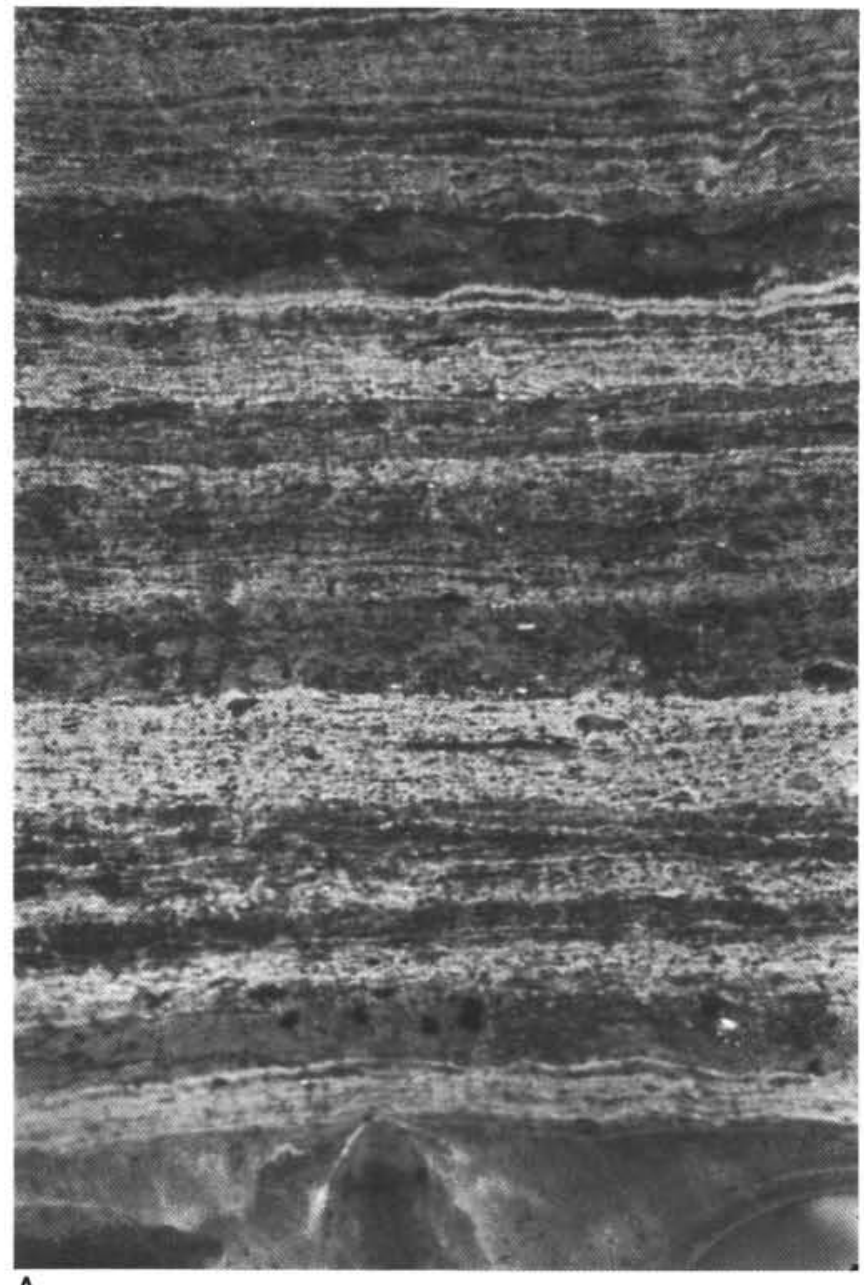

A

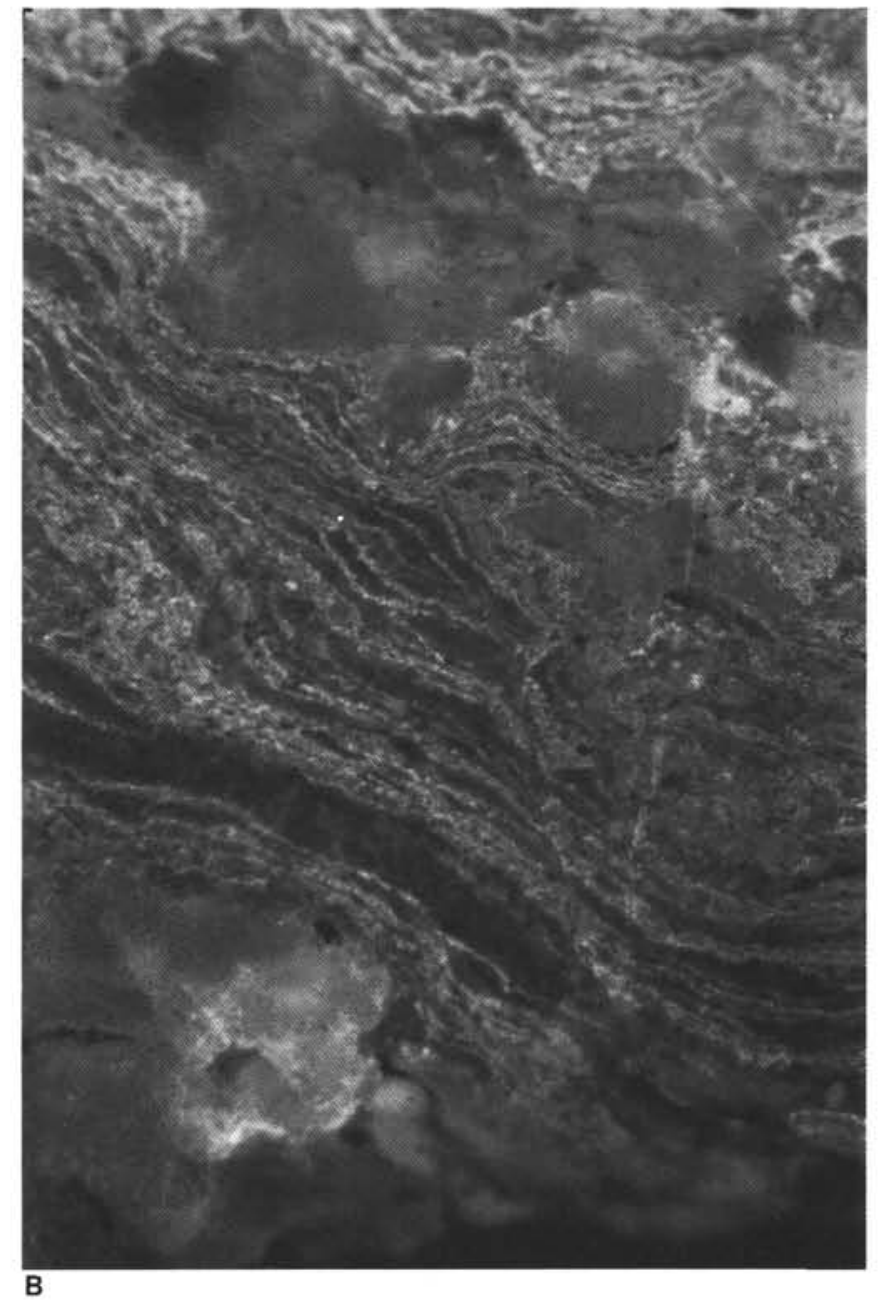

Figure 23. Photomicrographs (UV reflected light) of Interval 107-652A-64R-1, 103-107 cm. A. Contact of the finely laminated oil shale with underlying siliciclastic and authigenic grains. Length of lower edge corresponds to $0.6 \mathrm{~mm}$. Individual laminations appear bundled into clusters of 10 and more laminations. Remains of algae are not visible, and at higher magnification the organic matter appears amorphous and grainy. B. Contact of laminations with underlying clastic and chemical grains. Note the growth of laminae around detrital grains. This appearance resembles the growth pattern of a bacterial mat. 